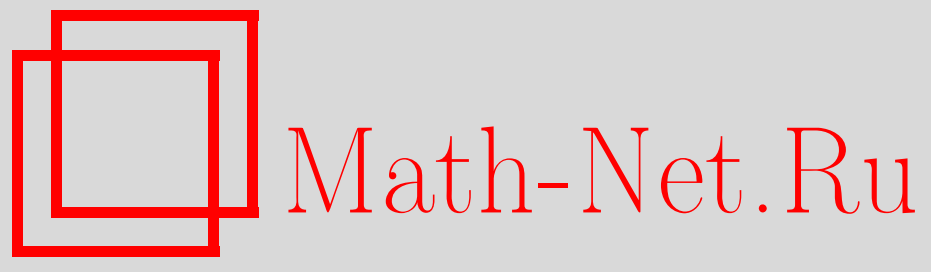

Ю. С. Осипов, Пакеты программ: подход к решению задач позиционного управления с неполной информацией, УМH, 2006, том 61, выпуск 4, 25-76

DOI: https://doi.org/10.4213/rm1760

Использование Общероссийского математического портала Math-Net.Ru подразумевает, что вы прочитали и согласны с пользовательским соглашением http://www . mathnet.ru/rus/agreement

Параметры загрузки:

IP : 54.237 .206 .68

26 апреля 2023 г., 16:39:30

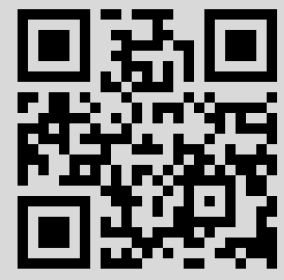




\section{Пакеты программ: \\ подход к решению задач позиционного управления с неполной информацией}

\section{Ю. С. Осипов}

Предлагается подход к решению задач позиционного управления динамической системой в условиях неполной информации о ее текущих фазовых состояниях. В основе подхода новое понятие пакетов программ процедур формирования управляющих программ, неупреждающих по отношению к динамике наблюдений. Для определенности подход реализуется применительно к конкретной задаче управления - задаче о гарантированном наведении управляемой системы на заданное целевое множество в фиксированный момент времени. Основной результат работы утверждает, что указанная задача разрешима в классе позиционных стратегий тогда и только тогда, когда она разрешима в классе пакетов программ. Тем самым указывается путь сведения позиционной постановки задач управления к программной. В этом смысле развивается методика программных конструкций, известная в теории позиционных дифференциальных игр.

Библиография: 30 названий.

\section{СОДЕРЖАНИЕ}

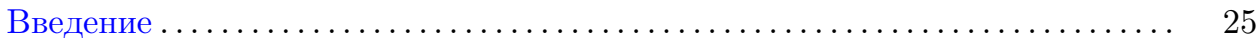

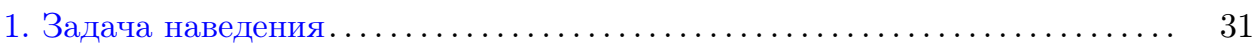

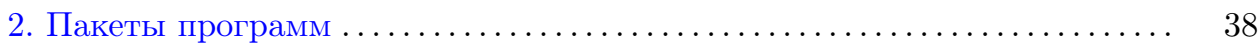

3. Стабильные мосты . . . . . . . . . . . . . . . . . . . . . . . 43

4. Информационные множества. Экстремальный сдвиг ............. 48

5. Решение задачи позиционного наведения .................... 55

6. Доказательство леммы о локальной оценке ................... 63

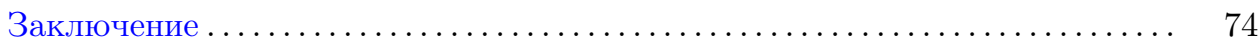

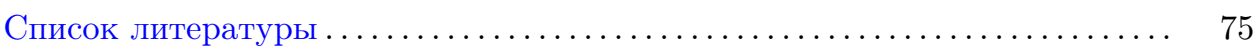

\section{Введение}

Математическая теория оптимального управления, созданная Л. С. Понтрягиным и его учениками [1], открыла новое мощное направление в тематике динамических систем, которое, с одной стороны, идейно перекликается с классическим вариационным исчислением, а с другой стороны, тесно связано

(C) Ю. С. Осипов, 2006 
с актуальной прикладной задачей - задачей о принятии оптимальных решений в динамике. Оригинальная и эффективная методика нахождения оптимальных управляющих акций, прежде всего знаменитый принцип максимума Понтрягина, предопределили бурное развитие этой тематики как в теоретическом русле, так и в контексте многочисленных приложений.

В своих изначальных постановках, задачи оптимального управления разрешаются в классе так называемых программных управлений - действий, порядок изменения которых во времени назначается (программируется) априори. Однако, параллельно с программными постановками, математическая теория оптимального управления выдвинула и важную задачу синтеза, в которой управляющие действия предлагается искать в форме обратных связей, т.е. в виде функций от текущих состояний динамической системы. Оптимальный синтез реализует оптимальную программу вне зависимости от выбора начального состояния системы.

Управление с обратной связью, известное также как позиционое управление, становится основным принципом управления для систем, подверженных неконтролируемым динамическим помехам. Изучение задач оптимизации систем с динамическим помехами в рамках теоретико-игрового формализма составляет, как известно, предмет теории дифференциальных игр. В ряде практически значимых случаев, лежащих у истоков теории дифференциальных игр, динамические помехи формируются стороной, целенаправленно препятствующей осуществлению целей управления, что подчеркивает игровую природу изучаемых процессов (см. [2]). Концепция дифференциальной игры получила сильное идейное и инструментальное развитие в работах Л. С. Понтрягина [3], [4]. Разработанная Н. Н. Красовским и его школой теория позиционных дифференциальных игр [5]-[12] окончательно вывела дифференциально-игровую тематику за рамки лишь антагонистического конфликта двух противоборствующих сторон, создав концепцию гарантирующего управления, согласно которой оптимизации подлежит гарантированный результат, достижимый при любых реализациях динамической помехи, вне зависимости от того, каким механизмом эти реализации формируются. Теория позиционных дифференциальных игр предъявляет широкий спектр технологий решения, среди которых особое место занимает универсальный метод управления с обратной связью - метод экстремального сдвига; с его помощью, в частности, устанавливаются центральные положения теории об альтернативной разрешимости взаимодополняющих задач о гарантирующем позиционном управлении.

Классические задачи теории дифференциальных игр, решаемые в классах обратных связей (позиционных стратегий управления), предполагают наличие у управляющей стороны полной информации о текущих фазовых состояниях динамической системы. Между тем, во многих практических задачах об управлении полная информация о фазовых состояниях принципиально недоступна. Типичными задачами такого рода выступают разнообразные задачи о преследовании одного маневрирующего объекта другим маневрирующим объектом, мотивированные инженерными (часто военного происхождения) проблемами автоматического управления. Неполнота информации о фазовых состояниях резко усложняет задачу, не давая, как правило, возможности воспользоваться при ее анализе основными результатами теории позиционных дифферециаль- 
ных игр. Поэтому при изучении отдельных, мотивированных приложениями, классов задач о позиционном управлении при неполной информации зачастую применяются специализированные методы, связанные с конкретными особенностями рассматриваемых постановок; в такого рода иследованиях речь обычно идет не об исчерпывающем решении задач, а о построении некоторых приемлемых, не обязательно оптимальных, стратегий управления (в этой связи, ограничиваясь сылками на работы школы Н. Н. Красовского, отметим, например, [13]-[15]).

В рамках позиционного дифференциально-игрового формализма, с использованием модификаций основополагающей конструкции - метода экстремального сдвига, проведен ряд успешных теоретических иследований задач управления с неполной информацией (см. гл. 15 в [6], а также [16]-[19]). Основное достижение этого цикла работ - установление теоретико-игровой двойственности между исходной задачей о гарантирующем управлении и дополняющей “информационной" контрзадачей, а также описание общих конструкций искомых позиционных стратегий при условии разрешимости исходной задачи. В [20] для теоретической характеризации задач с неполной информацией применяется метод динамического программирования.

Достаточно эффективными оказываются решения, основанные на методах устойчивого динамического обращения (динамической регуляризации) [21], [22]: с помощью этих методов алгоритмическая схема наблюдения-управления часто описывается до конца. Недостатком данного подхода является его ограниченность: условием успешного применения методов динамического обращения выступает, как правило, свойство так называемой идеальной наблюдаемости, предполагающее, что при известных историях управлений, помех и сигнала о состояниях однозначно восстановливается полное текущее состояние системы; отметим также специальные случаи, в которых методы динамического обращения приводят к искомым решениям в отсутствие свойства идеальной наблюдаемости (см. [23], [24]).

В контексте настоящей работы необходимо кратко остановиться на одном из основных инструментов теории позиционных дифференциальных игр - программных конструкциях. Програмные конструкции суть связующее звено между классическими формализмами теории оптимального управления и теории дифференциальных игр - програмным управлением и управлением по принципу обратной связи. Класс всех программных управлений много беднее класса всех обратных связей, в котором требуется искать законы игрового управления. Это обстоятельство, вместе с необходимостью, в игровых постановках, бороться с непредсказуемыми динамическими помехами, делает задачи об игровом управлении с обратной связью (об игровом позиционном управлении) "на порядок" сложнее задач о программном управлении. Кроме этого (и в значительной степени в связи с этим), для задач о программном управлении в рамках теории оптимального управления разработан мощный аналитический аппарат. Поэтому в теории позиционных дифференциальных игр чрезвычайную актуальность имеет следующий вопрос: возможно ли - и если да, то при каких условиях - свести исходную задачу об игровом позиционном управлении к той или иной задаче о программном управлении - пусть даже и при наличии программно действующего противника? 
Центральный метод сведения игровой задачи о позиционном управлении к (игровой же) задаче о программном управлении известен как метод экстремального прицеливания или метод программного максимина Н. Н. Красовского [5], [6]. Согласно этому методу, задача о программном управлении, эквивалентная исходной игровой задаче о позиционном управлении, конструируется, как задача о программном максимине. По условиям последней задачи, управляющей стороне предоставляется право узнать назначенную противной стороной программную реализацию динамической помехи и, в ответ на эту реализацию, выбрать свою программу управления. В весьма упрощенной интерпретации, позиционное управление по методу экстремального прицеливания состоит в следующем: в каждый момент времени управляющая сторона, рассмотрев текущую (связанную с текущим состоянием системы) задачу о программном максимине, строит свою оптимальную программу - ту, которая отвечает на максиминную ("наихудшую") программу противника; затем управляющая сторона фиксирует "начальное" управляющее действие, предписываемое построенной оптимальной программой на текущий момент времени, и применяет это управляющее действие к системе в данный текущий момент времени. Процесс повторения таких "начальных" управляющих действий, диктуемых постоянно пересчитываемыми оптимальными программами, обеспечивает управляющей стороне результат, никак не худший, чем тот, который гарантируется ею в задаче на программный максимин в исходной позиции. Достаточно ясно, что этот результат неулучшаем в классе всех позиционных стратегий управляющей стороны. Условием применимости метода экстремального прицеливания служит так называемое условие регулярности, которое, в самой грубой интерпретации, требует единственности максиминной программы противника. Хорошо известные метод стабильных дорожек и метод стабильных многообразий [6] также позволяют решать задачи об игровом позиционном управлении с помощью решения подходящих задач о программном управлении. Эти конструктивные, хотя и ограниченного применения, методы базируются на определенных "универсальных" мгновенных реакциях управляющего действия на текущие значения динамических помех.

Масштабным обобщением метода программного максимина выступает метод стохастического программного синтеза Н. Н. Красовского [7]. В отличие от метода программного максимина, действие которого ограничено условием регулярности, метод стохастического программного синтеза универсален. Суть обобщения состоит в том, что вместо детерминированных программ-"вызовов" противника и программ-“ответов” управляющей стороны рассматриваются стохастические программы, генерируемые расширяющимся во времени потоком случайных событий. Вводится математическое ожидание критерия оптимальности и с его помощью составляется стохастический программный максимин. Центральный пункт теории стохастического программного синтеза - утверждение о равенстве стохастического программного максимина и оптимального гарантированного результата в исходной, детерминированной, игровой задаче позиционного управления.

Идея о связи дифференциально-игровой концепции с концепцией программ как "ответов" на программы-“вызовы" противной стороны, эффективно реализованная в методе программного максимина, заложена также в известном ак- 
сиоматическом подходе к дифференциальным играм [25], [26]. Согласно данному подходу, действия управляющей стороны с самого начала понимаются как программные "ответы" на программные “вызовы” противника; при этом на программные "ответы" накладывается условие неупреждаемости (известное также как условие физической реализуемости, вольтерровости или динамичности). Условие неупреждаемости требует, чтобы программы-“ответы" одинаково реагировали на программы-“вызовы” до тех пор, пока программы-“"вызовы” не станут различаться между собой. Содержательно это условие означает, что управляющая сторона не может предвидеть (упреждать) будущее развитие помехи и принимает текущие решения на основании текущих историй. Отметим, что неупреждающие программные операторы “вызов"-“ответ", представляющие основу данного аксиоматического подхода, подпадают под определение операторов со свойством вольтерровости, данное А.Н. Тихоновым [27]. В [12], [7] неупреждающие операторы “вызов"-“ответ" рассматривались в рамках теории позиционных дифференциальных игр. В [12] представлен принципиальный факт об эквивлентной разрешимости игровых задач управления, поставленных в классе позиционных стратегий и в классе неупреждающих программных операторов - квазистратегий. Примечательно, что упомянутые выше метод стабильных дорожек и метод стабильных многообразий - это, в известном смысле, реализации некоторых специальных неупреждающих программных операторов. Отметим, наконец, что неупреждающие операторы, в контексте, весьма близком к теоретико-игровому, использовались в теории динамического обращения [21].

Настоящая работа обозначает новый подход, направленный на соединение задач позиционного управления с неполной информацией как объекта исследования с неупреждающими программными операторами как средством анализа. Несмотря на то, что задачи позиционного управления с неполной информацией, как отмечалось выше, традиционно связываются с игровыми ситуациями, в которых управляемая система подвергается действию неопределенных (либо противодействующих целям управления) динамических помех, мы здесь отказывается от этой традиции: изучаем управляемую систему, не подверженную действию динамических помех, возвращаясь, по сути, к задачам теории оптимального управления. Мы принимаем это упрощение из двух соображений. Во-первых, мы хотим наглядно показать, что неполнота информации о фазовых состояниях делает программную и позиционную постановки задачи о гарантирующем управлении неравносильными и при отсутствии динамических помех; таким образом, неопределенность в виде неполноты фазовой информации имеет принципиально иную природу, нежели неопределенность, возникающая вследствие действия динамических помех. Во-вторых, мы стремимся максимально облегчить изложение, сосредочившись на обработке только неопределенности, вызываемой неполнотой информации. Отметим, что техническая часть анализа оказывается достаточно трудоемкой даже в рассматриваемом упрощенном случае; подключение динамических помех видится как следующий, достаточно серьезный, шаг в предлагаемом направлении исследований.

Центральным понятием в излагаемом подходе является понятие пакета программ. Пакет программ - это, по содержанию, аналог неупреждающего программного оператора из теории дифференциальных игр. Аналогия, однако, 
далеко не прямая. Как адекватно перенести определение неупреждающего программного оператора на рассматриваемый здесь принципиально "неигровой" случай, с первого взгляда непонятно. Если говорить точнее, то с первого взгляда неясно, на какие "вызовы" следует заставить отвечать (программно) управляющую сторону: традиционные программы-“вызовы” (программы динамических помех) в рассматриваемом случае отсутствуют. Смысл задачи склоняет к тому, что "вызовом", подлежащим "ответу" в виде программы управления, должна быть история наблюдения сигнала о состояниях. Но история наблюдения сама определяется программой управления, на этом пути возникает порочный круг: "ответ" (программа управления) предопределяет "вызов" (историю наблюдения). Предлагаемое определение пакета программ устраняет этот парадокс путем введения подходящей “внешней” параметризации: пакет программ представляется как семейство неупреждающих программных "ответов" на все гипотетически возможные начальные состояния системы и на все гипотетически возможные реализации помех в канале наблюдения фазовых состояний.

Основной результат работы - утверждение о том, что задача о гарантирующем управлении в классе позиционных стратегий разрешима тогда и только тогда, когда она разрешима в классе пакетов программ. При обосновании этого факта мы существенно опираемся на одну из центральных идей теории позиционных дифференциальных игр - идею экстремального сдвига [6]. Нахождение разрешающего пакета программ - сложная, но все же программная задача; поэтому полученное утверждение об эквивалентности позиционной и пакетной постановок открывает, по крайней мере в принципе, возможности для аналитического описания условий разрешимости исходной задачи - о гарантирующем позиционном управлении в условиях неполной информации. В целях компактности изложения мы рассматриваем только один вариант задачи - задачу наведения, в которой управляемую систему требуется в фиксированный момент времени навести на фиксированное целевое множество.

Работа построена следующим образом.

В разделе 1, в предположении о неполноте информации о фазовых состояниях управляемой системы, описываются задача программного наведения и задача позиционного наведения на целевое множество. Приводятся примеры, показывающие, что условия разрешимости последней задачи, вообе говоря, шире. Далее изучается задача позиционного наведения.

В разделе 2 вводятся в рассмотрение пакеты программ и с их помощью ставится задача пакетного наведения. Устанавливается, что неразрешимость задачи пакетного наведения влечет за собой неразрешимость задачи позиционного наведения. Дальнейший материал работы посвящен обращению этого утверждения.

В разделе 3 с каждым пакетом программ связываются его так называемые фазовые образы - меняющиеся во времени классы множеств в пространстве состояний, достижимых при реализации программ из пакета. Семейство фазовых образов трактуется как аналог одной из несущих конструкций метода экстремального сдвига из теории позиционных дифференциальных игр - стабильного моста. 
В разделе 4 вводится локальное управление экстремального сдвига и формулируется ключевое техническое утверждение - лемма о верхней локальной оценке отклонения системы от стабильного моста при применении к системе управления экстремального сдвига.

В разделе 5 на базе управлений экстремального сдвига определяется позиционная стратегия экстремального сдвига. С помощью леммы о локальной оценке доказывается, что эта стратегия удерживает систему вблизи стабильного моста. В том случае, когда стабильные мосты порождены пакетами программ, решающими задачу пакетного наведения, данное свойство влечет вывод о том, что связанные с этими стабильными мостами стратегии экстремального сдвига решают задачу позиционного наведения. Последнее, вместе с результатом раздела 2, ведет к заключительному утверждению об эквивалентности задачи пакетного наведения и задачи позиционного наведения.

Раздел 6 посвящен доказательству основной леммы о локальной оценке из раздела 4.

Заключение кратко суммирует результаты и обозначает некоторые перспективы исследования.

Завершая введение, оговоримся, что данный выше обзор проблематики, связанной с задачами управления в условиях неполной информации, далеко не полон. В частности, опущены упоминания о соответствующих разделах теории $H_{\infty}$-управления, отсутствуют ссылки на исследования в области игр поиска, за рамками изложения остались важные примыкающие работы, касающиеся теории и методов оценивания. Эти ограничения продиктованы, в первую очередь, тем, чтобы с достаточной степенью ясности обозначить основные взаимосвязи между программными и позиционными подходами к задачам управления и, тем самым, обозреть исторический задел, непосредственно предшествующий исследованию, которое предпринято в настоящей работе.

\section{1. Задача наведения}

Будем рассматривать управляемую динамическую систему, описываемую обыкновенным дифференциальным уравнением

$$
\dot{x}(t)=f(t, x(t), u(t))
$$

здесь $t$ - время, меняющееся на ограниченном отрезке $\left[t_{0}, \vartheta\right]$ ненулевой длины, $x(t)$ - $n$-мерный вектор состояния системы в момент $t, u(t)$ - значение $m$-мерного управляющего вектора в этот момент. Функцию $f(\cdot):\left[t_{0}, \vartheta\right] \times \mathbb{R}^{n} \times \mathbb{R}^{m} \mapsto$ $\mathbb{R}^{n}$ считаем непрерывной. Следуя терминологии теории позиционного управления [6], программным управлением или, кратко, программой (для системы (1.1)) будем называть всякую измеримую по Лебегу функцию $u(\cdot):\left[t_{0}, \vartheta\right] \mapsto$ $P$; здесь $P$ - заданный компакт в $\mathbb{R}^{m}$, описывающий мәновенный ресурс управления. Множество всех программ будем обозначать символом $\mathscr{U}$. При заданном начальном состоянии

$$
x\left(t_{0}\right)=x_{0}
$$

системы со всякой программой $u(\cdot)$ связывается определенное на $\left[t_{0}, \vartheta\right]$ решение (Каратеодори) $x(\cdot)$ дифференциального уравнения (1.1) с начальным 
условием (1.2); такое решение будем называть движением (системы (1.1)) из начального состояния $x_{0}$ под действием программы $u(\cdot)$.

Формированием программы управления занимается некое лицо, которое будем называть управляющей стороной. Предполагаем, что управляющей стороне известно как уравнение (1.1) системы, так и ресурс $P$ управления. Доступная же управляющей стороне информация о состояниях $x(t)$ системы неполна. Именно, до начального момента $t_{0}$ движения управляющей стороне известен некоторый компакт $X_{0} \subset \mathbb{R}^{n}$, заведомо содержащий истинное начальное состояние $x\left(t_{0}\right)=x_{0}$ системы. В течение процесса управления в каждый момент $t \in\left[t_{0}, \vartheta\right]$ управляющей стороне поставляется результат $y(t)$ наблюдения $r$-мерного сигнала $s(x(t))$ о текущем состоянии $x(t)$ системы. Результат наблюдения не вполне точен, т.е. представляется в виде

$$
y(t)=s(x(t))+\xi(t)
$$

где $\xi(t)$ - помеха наблюдения, подчиненная ограничению $|\xi(t)| \leqslant h$; малый параметр $h$ - характеристика точности наблюдения; здесь и далее $|\cdot|$ - норма в конечномерном евклидовом постранстве. Сигнальная функиия $s(\cdot): \mathbb{R}^{n} \mapsto \mathbb{R}^{r}$, которая предполагается непрерывной, и точность наблюдения $h$ управляющей стороне известны.

В первом приближении, задача управляющей стороны состоит в формировании такой программы управления, которая гарантирует попадание состояния $x(\vartheta)$ системы в конечный момент времени $\vartheta$ на заданное иелевое множество $M \subset \mathbb{R}^{n}$. Придерживаясь концепции, принятой в теории позиционного управления [6], мы будем иметь дело со слегка ослабленной, асимптотической, постановкой задачи - будем требовать гарантии того, что состояние $x(\vartheta)$ системы может быть приведено в сколь угодно малую, заранее заданную, окрестность целевого множества: $x(\vartheta) \in[M]^{\varepsilon}$ со сколь угодно малым, заранее заданным, $\varepsilon>0$. Здесь и далее $[M]^{\varepsilon}-\varepsilon$-окрестность множества $M$, т.е. объединение открытых $\varepsilon$-окрестностей всех точек $x \in M$.

Данную задачу управления назовем задачей наведения (на целевое множество $M$ в момент $\vartheta$ ). Задачу наведения будем исследовать при следующих стандартных предположениях:

(A1) для всяких $x_{0} \in X_{0}$ и $u(\cdot) \in \mathscr{U}$ существует единственное движение $x(\cdot)$ из $x_{0}$ под действием $u(\cdot)$; последнее движение часто будем обозначать через $x\left(\cdot \mid x_{0}, u(\cdot)\right)$;

(A2) существует открытое ограниченное множество $X \subset \mathbb{R}^{n}$ такое, что $x\left(t \mid x_{0}, u(\cdot)\right) \in X$ при всех $t \in\left[t_{0}, \vartheta\right], x_{0} \in X_{0}, u(\cdot) \in \mathscr{U}$;

(A3) на множестве $X$ функция $f(\cdot)$ (см. (1.1)) удовлетворяет условию Липшица по переменной состояния: при некотором $L \geqslant 0$ имеем $\mid f\left(t, x_{1}, u\right)-$ $f\left(t, x_{2}, u\right)|\leqslant L| x_{1}-x_{2} \mid$ для всех $t \in\left[t_{0}, \vartheta\right], x_{1}, x_{2} \in X, u \in P$.

Рассмотрим сначала программную постановку задачи, в которой управляющая сторона формирует свою программу управления, используя лишь априорную информацию - множество $X_{0}$ возможных начальных состояний - и не принимает во внимание результаты $y(t)(1.3)$ наблюдений, поступающих по ходу управления системой. В программной постановке задача наведения - назовем ее задачей программного наведения - выглядит следующим образом: для 
каждого $\varepsilon$ найти программу $u(\cdot)$ такую, что $x\left(\vartheta \mid x_{0}, u(\cdot)\right) \in[M]^{\varepsilon}$ при всяком $x_{0} \in X_{0}$; программу $u(\cdot)$ с этим свойством будем называть $\varepsilon$-наводящей.

ЗАмЕчание 1.1. В случае, когда целевое множество $M$ замкнуто и при каждых $t \in\left[t_{0}, \vartheta\right], x \in X$ вектограмма $f(t, x, P)$ выпукла, разрешимость задачи программного наведения в указанной выше асимптотической постановке равносильна разрешимости задачи точного программного наведения, т.е. существованию 0-наводящей программы. Это следует из того, что в указанном случае множество всех движений $\mathscr{X}=\left\{x\left(\cdot \mid x_{0}, u(\cdot)\right): x_{0} \in X_{0}, u(\cdot) \in \mathscr{U}\right\}$ есть компакт в $C\left(\left[t_{0}, \vartheta\right], \mathbb{R}^{n}\right)$. В общем же случае такая равносильность не имеет места. Например, легко видеть, что для двумерной системы

$$
\dot{x}^{(1)}(t)=u(t), \quad \dot{x}^{(2)}(t)=\left|x^{(1)}(t)\right|
$$

на $\left[t_{0}, \vartheta\right]=[0,1]$ с двухточечным ресурсом управления $P=\{-1,1\}$, одноэлементным множеством $X_{0}=\{0,0\}$ начальных состояний и целевым множеством $M=X_{0}$ 0-наводящей программы не существует, а задача программного наведения разрешима.

Если задача программного наведения разрешима, то понятно, что управляющая сторона, назначив подходящим образом программу управления, априори гарантирует попадание системы в конечный момент времени $\vartheta$ в произвольную, заранее заданную, окрестность целевого множества $M$ независимо от того, каким образом истинное начальное состояние $x_{0}$ системы реализовано в пределах множества $X_{0}$. Если же задача программного наведения не разрешима, то управляющая сторона априори такой гарантии дать не может. Действительно, если задача программного наведения не разрешима, то при любом априорном выборе управляющей стороной программы управления $u(\cdot)$ в ответ на этот выбор отыщется такое "неблагоприятное" начальное состояние $x_{0} \in X_{0}$, что реализуемое на движении $x(\cdot)=x\left(\cdot \mid x_{0}, u(\cdot)\right)$ состояние $x(\vartheta)$ попадет во внешность $\varepsilon_{*}$-окрестности целевого множества $M$ с некоторым фиксированным положительным $\varepsilon_{*}$. Но управляющая сторона, выбравшая программу $u(\cdot)$, априори не может исключить того, что именно это, "неблагоприятное" для $u(\cdot)$, начальное состояние $x_{0}$ является истинным; значит, управляющая сторона не в состоянии априори гарантировать наведение системы на $\varepsilon_{*}$-окрестность целевого множества.

Следуя подходу теории позиционного управления, рассмотрим также позиционную постановку задачи наведения, в которой управляющая сторона формирует программу управления адаптивно, с учетом текущих результатов наблюдения $y(t)(1.3)$. Допустимые механизмы адаптивного формирования программ управления управляющей стороной будем называть позиционными стратегиями. Позиционная стратегия складывается из последовательности моментов $\tau_{i} \in\left[t_{0}, \vartheta\right]$ корректировки управления и последовательности обратных связей $U_{i}$ в моменты $\tau_{i}$. Обратная связь в момент $\tau_{i}$ - это принимаемое управляющей стороной правило по выработке в момент $\tau_{i}$ значения $u\left(\tau_{i}\right)$ управляющего параметра. Выработанное в момент $\tau_{i}$ значение $u\left(\tau_{i}\right)$ управляющая сторона применяет в качестве управления к системе в течение промежутка времени $\left[\tau_{i}, \tau_{i+1}\right)\left(u(t)=u\left(\tau_{i}\right)\right.$ подставляется в $(1.1)$ при всех $\left.t \in\left[\tau_{i}, \tau_{i+1}\right)\right)$. Значение $u\left(\tau_{i}\right)$ вырабатывается управляющей стороной в зависимости от сложившихся 
к моменту $\tau_{i}$ истории наблюдения $y(t)(1.3)$ и истории управления $u(t)$, которые (истории) представляются соответственно функцией $y_{\tau_{i}}(\cdot): t \mapsto y(t)$, определенной на $\left[t_{0}, \tau_{i}\right]$, и функцией $u_{\tau_{i}}(\cdot): t \mapsto u(t)$, определенной на $\left[t_{0}, \tau_{i}\right)$ : $u\left(\tau_{i}\right)=U\left(y_{\tau_{i}}(\cdot), u_{\tau_{i}}(\cdot)\right)$.

Прежде чем привести формальное определение позиционной стратегии, соответствующее данному выше содержательному описанию, введем вспомогательные множества, с помощью которых будем задавать области определения обратных связей. Для всякого $\tau \in\left(t_{0}, \vartheta\right]$ через $\mathscr{U}_{\tau}$ обозначим множество сужений на $\left[t_{0}, \tau\right)$ всех программ $u(\cdot) \in \mathscr{U}$; элементы множества $\mathscr{U}_{\tau}$ будем называть программами до момента $\tau$. Для формального удобства примем за $\mathscr{U}_{t_{0}}$ какоелибо одноэлементное подмножество множества $P$. Через $\mathscr{Y}_{\tau}$, где $\tau \in\left(t_{0}, \vartheta\right]$, будем обозначать множество всех функций $y(\cdot):\left[t_{0}, \tau_{i}\right] \mapsto \mathbb{R}^{r}$; элементы множества $\mathscr{Y}_{\tau}$ будем называть наблюдениями до момента $\tau$.

Позиционную стратегию (управляющей стороны) определим формально, как произвольную конечную последовательность

$$
S=\left(\tau_{i}, U_{i}\right)_{i=0}^{l+1},
$$

где $t_{0}=\tau_{0}<\cdots<\tau_{l+1}=\vartheta$ и, при каждом $i=0, \ldots, l+1, U_{i}$ - отображение произведения $\mathscr{Y}_{\tau_{i}} \times \mathscr{U}_{\tau_{i}}$ в ресурсное множество $P$; каждый момент $\tau_{i}$, где $i=$ $0, \ldots, l$, называем моментом корректировки (управления), а отображение $U_{i}-$ обратной связъю в момент $\tau_{i}$ (соответствующими позиционной стратегии $S$ ).

Приведенное выше неформальное описание действия позиционной стратегии ведет к следующему определению. Через $\Xi$ будем далее обозначать множество всех помех наблюдения, т.е. всех функций $\xi(\cdot):\left[t_{0}, \vartheta\right] \mapsto \mathbb{R}^{r}$, и через $\Xi^{h}$, где $h \geqslant 0,-$ множество всех помех наблюдения точности $h$, т.е. всех $\xi(\cdot) \in \Xi$ таких, что $|\xi(t)| \leqslant h\left(t \in\left[t_{0}, \vartheta\right]\right)$. Управляемым процессом с начальным состоянием $x_{0} \in X_{0}$ под действием позиционной стратегии $S$ (1.4) при помехе наблюдения $\xi(\cdot)$ будем называть тройку $(u(\cdot), x(\cdot), y(\cdot))$ такую, что $u(\cdot) \in \mathscr{U}$, $x(\cdot)=x\left(\cdot \mid x_{0}, u(\cdot)\right), y(t)=s(x(t))+\xi(t)\left(t \in\left[t_{0}, \vartheta\right]\right)$ и для каждого $i=0, \ldots, l$ выполняется

$$
u(t)=u\left(\tau_{i}\right)=U_{i}\left(y_{\tau_{i}}(\cdot), u_{\tau_{i}}(\cdot)\right) \quad\left(t \in\left[\tau_{i}, \tau_{i+1}\right)\right),
$$

где $u_{\tau_{i}}(\cdot)$ - сужение $u(\cdot)$ на $\left[t_{0}, \tau_{i}\right)$ и $y_{\tau_{i}}(\cdot)$ - сужение $y(\cdot)$ на $\left[t_{0}, \tau_{i}\right]$; функцию $u(\cdot)$ будем при этом называть программой, функцию $x(\cdot)-$ движением и функцию $y(\cdot)$ - наблюдением в управляемом процессе $(u(\cdot), x(\cdot), y(\cdot))$.

Понятно, что определенный выше управляемый процесс существует и единственен. Множество всех управляемых процессов с начальным состоянием $x_{0} \in X_{0}$ под действием позиционной стратегии $S$ при всевозможных помехах наблюдения $\xi(\cdot)$ размера $h\left(\xi(\cdot) \in \Xi^{h}\right)$ обозначим через $\mathscr{P}\left(x_{0}, S, h\right)$. В управляемых процессах нас будут интересовать, в первую очередь, компоненты движения. Множество движений (системы (1.1)) из начального состояния $x_{0} \in X_{0}$ под действием позиционной стратегии $S$ при параметре $h$ точности наблюдения определим как

$$
\mathscr{X}\left(x_{0}, S, h\right)=\left\{x(\cdot):(u(\cdot), x(\cdot), y(\cdot)) \in \mathscr{P}\left(x_{0}, S, h\right)\right\} .
$$

Управляющей стороне, при известном параметре $h$ точности наблюдения, надлежит так выбрать позиционую стратегию $S_{h}$, что, каково бы ни было 
начальное состояние $x_{0} \in X_{0}$, любое движение из множества движений $\mathscr{X}\left(x_{0}, S_{h}, h\right)$ в момент $\vartheta$ приводится в сколь угодно малую наперед заданную окрестность целевого множества $M$, если только точность наблюдения достаточно высока - параметр $h$ достаточно мал. Таким образом, мы будем иметь дело с семействами $\left(S_{h}\right)_{h>0}$ позиционых сратегий. Формально, будем говорить, что семейство $\left(S_{h}\right)_{h>0}$ позиционых сратегий - наводящее, если для любого $\varepsilon>0$ найдется $h_{0}>0$ такое, что при всяких $h \in\left[0, h_{0}\right]$ и $x_{0} \in X_{0}$ каждое движение $x(\cdot) \in \mathscr{X}\left(x_{0}, S_{h}, h\right)$ удовлетворяет условию $x(\vartheta) \in[M]^{\varepsilon}$. Задача позииионного наведения состоит в нахождении наводящего семейства позиционных стратегий.

ЗАмЕчАниЕ 1.2. Предложенная постановка задачи позиционного наведения, предполагающая устранение ошибки в достижении результата (состоящего в наведении системы на целевое множество) при бесконечном улучшении точности наблюдения, соответствует концепции регуляризации из теории некорректно поставленных задач [28]. В теории позиционых дифференциальных игр (с полной информацией) такого рода постановки стимулировали разработку специального класса законов позиционного управления - так называемых процедур управления с поводырем [6]. В нашем случае принятие "регуляризованной" постановки, допускающей малые шумы в канале наблюдения, мотивировано двумя, в известном смысле противоположными, обстоятельствами: во-первых, такая постановка более реалистична с точки зрения приложений, во-вторых, допущение малых шумов оказывается "выгодным" с технической точки зрения (наличие шумов существенно используется в разделе 6). Изучение "идеальной" постановки задачи, предполагающей идеально точное наблюдение сигнала (корректность, условия разрешимости и т. д.), выходит за рамки настоящей работы.

Итак, если задача позиционного наведения разрешима, то управляющая сторона, выбрав, при известном, достаточно малом, параметре $h$ точности наблюдения, соответствующую позиционную стратегию $S_{h}$ из наводящего семейства, априори гарантирует попадание системы в конечный момент времени $\vartheta$ в малую окрестность целевого множества независимо от того, как истинное начальное состояние $x_{0}$ системы реализовано в пределах множества $X_{0}$. Если же задача позиционного наведения не разрешима, то при любом параметpe $h$ точности наблюдения и любом априорном выборе управляющей стороной позиционной стратегии $S_{h}$ в ответ на этот выбор найдется такое "неблагоприятное" начальное состояние $x_{0} \in X_{0}$ и такое "неблагоприятное" движение $x(\cdot) \in \mathscr{X}\left(x_{0}, S_{h}, h\right)$, что реализуемое на этом движении состояние $x(\vartheta)$ попадет во внешность $\varepsilon_{*}$-окрестности целевого множества с некоторым фиксированным положительным $\varepsilon_{*}$. Но управляющая сторона априори не в состоянии исключить того, что именно эти, "неблагоприятные" для позиционной стратегии $S_{h}$, начальное состояние $x_{0}$ и движение $x(\cdot)$ будут реализованы в действительности. Таким образом, в этом случае управляющая сторона не может априори гарантировать наведение системы на $\varepsilon_{*}$-окрестость целевого множества путем выбора позиционной стратегии ни при каком параметре $h$ точности наблюдения. 
Нетрудно установить следующие два факта: во-первых, из разрешимости задачи программного наведения следует разрешимость задачи позиционного наведения; во-вторых, если множество $X_{0}$ одноэлементно, то верно и обратное; в последнем случае задачи программного и позиционного наведения эквивалентны. Эти факты суть простые следствия того, что множество всех кусочно-постоянных программ с конечным числом скачков плотно в множестве $\mathscr{U}$ всех программ в метрике пространства $L^{1}\left(\left[t_{0}, \vartheta\right], \mathbb{R}^{m}\right)[29]$ (доказательства опускаем). В общем случае, когда множество $X_{0}$ не одноэлементно, задача позиционного наведения может быть разрешимой при неразрешимости задачи программного наведения. Подобная ситуация возникает тривиально, если наблюдение, произведенное в начальный момент $t_{0}$, позволяет (при достаточно малом параметре $h$ точности) однозначно идентифицировать начальную точку $x_{0}$. Действительно, после такой идентификации можно "подстраивать" программу $u(\cdot)$ под начальную точку, что значительно расширяет возможности управления по сравнению с чисто программной постановкой, когда наблюдение, производимое в начальный момент, не принимается во внимание. Приведем простейший пример такого рода.

ПримеР 1.1. Рассмотрим одномерную управляемую систему

$$
\dot{x}(t)=u(t)
$$

на $\left[t_{0}, \vartheta\right]=[0,1]$ с ресурсом управления $P=[-1,1]$, двухточечным множеством $X_{0}=\{-1,1\}$ начальных состояний и целевым множеством $M=\{0\}$. Легко видеть, что при любой программе $u(\cdot)$ конечное состояние $x(1)$ системы, достигаемое вдоль одного из двух движений $x(\cdot \mid-1, u(\cdot))$ и $x(\cdot \mid 1, u(\cdot))$, отклоняется от 0 не менее, чем на 1 . Таким образом, задача программного наведения неразрешима. Рассмотрим задачу позиционного наведения. Пусть сигнальная функция $s(\cdot)$ имеет вид $s(x)=x$. Тогда при $|\xi(0)| \leqslant h<2$ наблюдение $y(0)=s\left(x_{0}\right)+\xi(0)$, произведенное в начальный момент 0 , позволяет различить начальные состояния: $y(0)<0$ означает, что $x_{0}=-1$, а $y(0) \geqslant 0$ означает, что $x_{0}=1$. При начальном состоянии -1 программа $u(t)=1$ приводит систему в момент 1 в целевое состояние 0, а при начальном состоянии 1 это делает программа $u(t)=-1$. Поэтому позиционная стратегия $S=\left(\tau_{i}, U_{i}\right)_{i=0}^{1}$ с единственным моментом корректировки $\tau_{0}=0$ и обратной связью $U_{0}\left(y_{0}(\cdot), u_{0}(\cdot)\right)=-\operatorname{sign}\left(y_{0}(0)\right)$ обеспечивает условие $x(1)=0 \in M$ для любого начального состояния $x_{0}$ и любого (в данном случае, как легко видеть, единственного) движения $x(\cdot) \in \mathscr{X}\left(x_{0}, S, h\right)$, если только $h<2$. Иначе говоря, одноэлементное семейство позиционных стратегий, единственным элементом которого служит позиционная стратегия $S$, является наводящим. Задача позиционного наведения разрешима.

Приведем пример неэквивалентности задач программного и позиционного наведения в нетривиальной ситуации, когда наблюдение любой точности, производимое в начальный момент времени, не позволяет различить начальные состояния.

ПРимеР 1.2. Рассмотрим одномерную управляемую систему

$$
\dot{x}(t)=\operatorname{sign}(x) \min \{|x|, 1\}+\max \{0, t\} u(t),
$$


функционирующую на отрезке времени $\left[t_{0}, \vartheta\right]=[-2,1]$, с ресурсом управления $P=[-1,1]$ и двухточечным множеством $X_{0}=\{-1,1\}$ начальных состояний. Система устроена так, что от момента -2 до момента 0 она движется "без управления", управление "плавно включается" начиная с момента 0 и при этом на участке "без управления" движения $x^{-}(\cdot)$ и $x^{+}(\cdot)$ системы, исходящие из начальных состояний -1 и 1 , "расходятся": $x^{-}(t)=-1-(t+2)$ и $x^{+}(t)=$ $1+(t+2)$. Таким образом, независимо от выбора программы $u(\cdot)$ для движения $x\left(\cdot \mid x_{0}, u(\cdot)\right)$ выполняется

$$
x\left(0 \mid x_{0}, u(\cdot)\right)= \begin{cases}-3, & \text { если } x_{0}=-1 \\ 3, & \text { если } x_{0}=1 .\end{cases}
$$

В качестве целевого множества возьмем $M=\{-9 / 2,9 / 2\}$. Тогда задача программного наведения не разрешима. Действительно, как видно из уравнения системы и из (1.6), при любой программе $u(\cdot)$

$$
x\left(1 \mid x_{0}, u(\cdot)\right)=\left\{\begin{array}{ll}
-4+r, & \text { если } x_{0}=-1, \\
4+r, & \text { если } x_{0}=1,
\end{array} \quad r=\int_{0}^{1} t u(t) d t \in[-1 / 2,1 / 2] .\right.
$$

При $r \geqslant 0$ имеем $x(1 \mid-1, u(\cdot)) \in[-8 / 2,-7 / 2]$ и, следовательно, $x(1 \mid-1, u(\cdot)) \notin$ $[M]^{1 / 2}$, при $r \leqslant 0$ выполняется $x(1 \mid 1, u(\cdot)) \in[7 / 2,8 / 2]$ и опять $x(1 \mid 1, u(\cdot)) \notin$ $[M]^{1 / 2}$. Таким образом, для всякой программы $u(\cdot)$ имеется начальное состояние $x_{0} \in X_{0}$ такое, что $x(1 \mid 1, u(\cdot)) \notin[M]^{1 / 2}$. Это говорит о неразрешимости задачи программного наведения.

Рассмотрим теперь задачу позиционного наведения при сигнальной функции $s(\cdot)$ вида

$$
s(x)= \begin{cases}x+2, & \text { если } x \leqslant-2, \\ 0, & \text { если } x \in[-2,2], \\ x-2, & \text { если } x \geqslant 2 .\end{cases}
$$

В отличие от предыдущего примера, наблюдение любой точности, произведенное в начальный момент -3, не позволяет различить начальные состояния: $s(-1)+\xi(-3)=s(1)+\xi(-3)$ при всяких $h>0$ и $\xi(\cdot) \in \Xi^{h}$. Наблюдение же точности $h<1$, произведенное в момент 0, позволяет это сделать: как видим из (1.6), $y(0)=s(x(0 \mid-1, u(\cdot)))+\xi(0)<0$ и $y(0)=s(x(0 \mid 1, u(\cdot)))+\xi(0)>0$ для всякой помехи наблюдения $\xi(\cdot)$ точности $h<1$ и всякой программы $u(\cdot)$. В первом случае, полагая $u(t)=-1$ при $t \geqslant 0$, получаем (см. (1.7)) $x(1 \mid-1, u(\cdot))=-9 / 2 \in M$, во втором случае, полагая $u(t)=1$ при $t \geqslant 0$, получаем $x(1 \mid 1, u(\cdot))=9 / 2 \in M$. Приходим к такому естественному способу управления: в начальный момент включаем любую - например, нулевую, программу и сохраняем ее до момента 0. В момент 0 определяем знак сигнала $y(0)=s(x(0))+\xi(0)$. Если $y(0)<0$, включаем программу $u(t)=-1$, если $y(0)>0$, включаем программу $u(t)=1$. Этот способ управления реализуется позиционной стратегией $S=\left(\tau_{i}, U_{i}\right)_{i=0}^{2}$ с двумя моментами корректировки, $\tau_{0}=-3$ и $\tau_{1}=0$, и обратными связями $U_{0}\left(y_{-3}(\cdot), u_{-3}(\cdot)\right)=0$ и $U_{1}\left(y_{0}(\cdot), u_{0}(\cdot)\right)=\operatorname{sign}\left(y_{0}(0)\right)$. Таким образом, одноэлементное семейство позиционных стратегий, единственным элементом которого служит позиционная стратегия $S$, является наводящим. Задача позиционного наведения разрешима. 
Итак, единственный фактор, делающий позиционную постановку задачи наведения сильнее программной, - это неоднозначность в задании начального состояния. Далее мы сконцентрируемся на задаче позиционного наведения. Наша цель - получить условия разрешимости этой задачи и указать общую конструкцию наводящего семейства позиционных стратегий.

\section{2. Пакеты программ}

Основной конструкцией в последующем анализе будут специальные семейства программ, параметризованные начальными точками $x_{0} \in X_{0}$ и функциями $\xi(\cdot)$, доставляющими помехи в наблюдениях. Такие семейства будем называть пакетами программ. Общий элемент пакета программ будем обычно обозначать $u(\cdot)_{x_{0}, \xi(\cdot)}$.

Понятие пакета программ смыкается с используемыми в теории дифференциальных игр понятиями квазистратегии и $Q$-процедуры [12], [7]. По своему содержанию квазистратегии и $Q$-процедуры - это идеализированные правила неупреждающего программного ответа "игрока-союзника" на заранее не известные ему программы "игрока-противника". Аналоги этих правил, пакеты про-

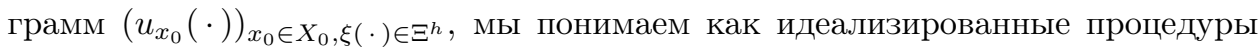
формирования неупреждаюего программного ответа на поступающие результаты $y(t)$ наблюдения. Заметим, что при фиксированной программе $u(\cdot)$ peзультаты наблюдения $y(t)$ определяются двумя параметрами - начальным состоянием $x_{0}$ и помехой наблюдения $\xi(\cdot)$. Поэтому, в известной мере "обращая" ситуацию, программу, реагирующую на результаты наблюдения, мы задаем через ее зависимость от этих, определяющих, параметров: $u(\cdot)=u_{x_{0}, \xi(\cdot)}(\cdot)$. Назначая такие программные реакции заранее и собирая их в единое семейство, приходим к предлагаемому понятию пакета программ. В отличие от позиционных стратегий, пакеты программ не рассчитаны на то, чтобы служить моделями реальных алгоритмов управления; они имеют искусственный характер и играют техническую роль. Тем не менее (подобно квазистратегиям в теории дифференциальных игр), пакеты программ как процедуры управления имеют весьма прозрачную содержательную интерпретацию.

Остановимся на такой интерпретации: опишем процедуру управления, задаваемую пакетом программ $\left(u_{x_{0}}(\cdot)\right)_{x_{0} \in X_{0}, \xi(\cdot) \in \Xi^{h}}$, неформально. Попутно, на содержательном уровне, подойдем к характеристическому для пакетов программ "условию неупреждаемости". Для простоты рассмотрим пока процесс принятия решения управляющей стороной в начальный момент $t_{0}$. В этот момент управляющая сторона получает информацию о расположении истинного начального состояния $\widehat{x}_{0}$ в виде результата наблюдения $y\left(t_{0}\right)=s\left(\widehat{x}_{0}\right)+\widehat{\xi}\left(t_{0}\right)$, где $\widehat{\xi}\left(t_{0}\right)$ - значение в момент $t_{0}$ неизвестной истинной помехи наблюдения $\widehat{\xi}(\cdot) \in \Xi^{h}$. На основании этой информации управляющая сторона выделяет множество всех "потенциально истинных" пар $\left(x_{0}, \xi(\cdot)\right)$ "начальное состояие помеха наблюдения", т.е. таких пар $\left(x_{0}, \xi(\cdot)\right)$, которые совместимы с результатом наблюдения $y\left(t_{0}\right)$. Понятно, что пара $\left(x_{0}, \xi(\cdot)\right)$ "потенциально истинна", если $x_{0} \in X_{0}, \xi\left(t_{0}\right) \in \Xi^{h}$ и $s\left(x_{0}\right)+\xi\left(t_{0}\right)=y\left(t_{0}\right)$. В момент $t_{0}$ управляющая сторона применяет в качестве управления значение $u_{x_{0}, \xi(\cdot)}\left(t_{0}\right)$ той программы из пакета, которая отвечает на какую-либо из "потенциально истинных" пар 
$\left(x_{0}, \xi(\cdot)\right)$ (ради ясности мы игнорируем тот факт, что значение управляющего параметра в один, отдельно взятый момент времени не влияет на развитие движения системы). Но для управляющей стороны все "потенциально истинные" пары $\left(x_{0}, \xi\left(t_{0}\right)\right)$ в равной мере подозрительны на то, чтобы быть реальной парой $\left(\widehat{x}_{0}, \widehat{\xi}\left(t_{0}\right)\right)$, значит, у управляющей стороны нет оснований для того, чтобы на различные "потенциально истинные" пары $\left(x_{0}, \xi(\cdot)\right)$ отвечать по-разному. Это ведет к тому естественному ограничению, что значения $u_{x_{0}, \xi(\cdot)}\left(t_{0}\right)$ должны быть одинаковыми для всех "потенциально истинных" пар $\left(x_{0}, \xi(\cdot)\right)$. Более точно это ограничение можно записать так: если $s\left(x_{0}\right)+\xi\left(t_{0}\right)=s\left(\bar{x}_{0}\right)+\bar{\xi}\left(t_{0}\right)$ (где $\left.x_{0}, \bar{x}_{0} \in X_{0}, \xi(\cdot), \bar{\xi}(\cdot) \in \Xi^{h}\right)$, то $u_{x_{0}, \xi(\cdot)}\left(t_{0}\right)=u_{\bar{x}_{0}, \bar{\xi}(\cdot)}\left(t_{0}\right)$.

В основе последнего ограничения лежит гипотеза неупреждаемости - невозможности предугадывать будущее и пересматривать ранее принятые решения. Поясним это соображение (мы, разумеется, остаемся в рамках неформального изложения). Важно заметить, что некоторые пары $\left(x_{0}, \xi(\cdot)\right)$, "потенциально истинные" на момент $t_{0}$, по ходу процесса управления могут стать "ложными" - несовместимыми с результатами $y(t)=s(\widehat{x}(t))+\widehat{\xi}(t)$ наблюдения реальных состояний $\widehat{x}(t)$ при реальных значениях $\widehat{\xi}(t)$ помехи $\left(t \in\left[t_{0}, \vartheta\right]\right)$. Это происходит тогда, когда состояние $x(t)$, в которое переходит, в силу уравнения (1.1) (под действием реализованного управления), начальная точка $x_{0}$, и значение $\xi(t)$ помехи порождают сигнал $s(x(t))+\xi(t)$, отличный от наблюдаемого значения $y(t)$. После вычеркивания "ложных пар" исходное множество "потенциально истинных" пар сужается: получается уменьшенное множество пар $\left(x_{0}, \xi(\cdot)\right)$, "потенциально истинных" на момент $t$. Предположим теперь, что управляющей стороной для различных пар $\left(x_{0}, \xi(\cdot)\right)$, "потенциально истинных" на начальный момент $t_{0}$, запланированы различные значения $u_{x_{0}, \xi(\cdot)}\left(t_{0}\right)$ управляющего параметра. Одно из них, $u_{\bar{x}_{0}, \bar{\xi}(\cdot)}\left(t_{0}\right)$, отвечающее некоторой паре $\left(\bar{x}_{0}, \bar{\xi}(\cdot)\right)$, “потенциально истинной" на момент $t_{0}$, применено на самом деле. Вполне может произойти так, что в какой-то момент $t>t_{0}$ пара $\left(\bar{x}_{0}, \bar{\xi}(\cdot)\right)$ окажется “ложной". Пусть это действительно произошло. Тогда в момент $t$ управляющая сторона вынуждена признать, что в прошлом, в момент $t_{0}$, ею принято неверное решение, ориентированное на "ложную" пару $\left(\bar{x}_{0}, \bar{\xi}(\cdot)\right)$. У управляющей стороны возникает потребность изменить это решение - вместо $u_{\bar{x}_{0}, \bar{\xi}(\cdot)}\left(t_{0}\right)$ в момент $t_{0}$ применить $u_{x_{0}, \xi(\cdot)}\left(t_{0}\right)$, соответствующее какой-либо паре $\left(x_{0}, \xi(\cdot)\right)$, которая на момент $t$ "потенциально истинна". Однако выбрать “верное" $u_{x_{0}, \xi(\cdot)}\left(t_{0}\right)$ вместо "неверного" $u_{\bar{x}_{0}, \bar{\xi}(\cdot)}\left(t_{0}\right)$ разрешается никак не позже момента $t_{0}$, такой выбор, следовательно, осуществим лишь при возможности у управляющей стороны предвидеть (упредить) будущее развитие событий. Если отказаться от такой возможности и оставаться в рамках физически реализуемых, неупреждающих, схем принятия решений, то следует признать, что допущение для различных пар $\left(x_{0}, \xi(\cdot)\right)$, “потенциально истинных" на момент $t_{0}$, различных значений $u_{x_{0}, \xi(\cdot)}\left(t_{0}\right)$ управляющего параметра выводит за рамки таких схем. Таким образом, применительно к начальному моменту времени $t_{0}$, условие равенства между собой значений $u_{x_{0}, \xi(\cdot)}\left(t_{0}\right)$ для всех "потенциально истинных" на момент $t_{0}$ пар $\left(x_{0}, \xi(\cdot)\right)$ есть условие неупреждаемости рассматриваемой процедуры управления.

Аналогичные соображения, примененные к произвольному моменту времени $\tau \in\left[t_{0}, \vartheta\right]$, влекут следующее “ограничение неупреждаемости”: если на отрезке 
времени $\left[t_{0}, \tau\right]$ значения управления сформированы программой $u_{\bar{x}_{0}, \bar{\xi}(\cdot)}(\cdot)$, отвечающей некоторой “потенциально истинной” на момент $\tau$ паре $\left(\bar{x}_{0}, \bar{\xi}(\cdot)\right)$, то на отрезке $\left[t_{0}, \tau\right]$ программа $u_{\bar{x}_{0}, \bar{\xi}(\cdot)}(\cdot)$ неотличима от программы $u_{x_{0}, \xi(\cdot)}(\cdot)$, отвечающей любой другой паре $\left(x_{0}, \xi(\cdot)\right)$, также “потенциально истинной" на момент $\tau,-$ такой, что исходящее из $x_{0}$ движение, порожденное фактически реализованным до момента $\tau$ отрезком программы $u_{\bar{x}_{0}, \bar{\xi}(\cdot)}(\cdot)$, совместимо с фактическими результатами наблюдения при помехе $\xi(\cdot)$.

Перейдем к формальному определению. Пару $\left(x_{0}, \xi(\cdot)\right) \in X_{0} \times \Xi$ назовем совместимой с тройкой $\left(\bar{x}_{0}, \bar{\xi}(\cdot), \bar{u}(\cdot)\right) \in X_{0} \times \Xi \times \mathscr{U}$ до момента $\tau \in\left[t_{0}, \vartheta\right]$, если

$$
s\left(x\left(t \mid x_{0}, \bar{u}(\cdot)\right)\right)+\xi(t)=s\left(x\left(t \mid \bar{x}_{0}, \bar{u}(\cdot)\right)\right)+\bar{\xi}(t)
$$

при всех $t \in\left[t_{0}, \tau\right]$. При каждом $h \geqslant 0$ семейство $\left(u_{x_{0}, \xi(\cdot)}(\cdot)\right)_{x_{0} \in X_{0}, \xi(\cdot) \in \Xi^{h}}$ программ будем называть пакетом программ точности $h$, если оно удовлетворяет следующему условию неупреждаемости: для всяких $\bar{x}_{0} \in X_{0}$, $\bar{\xi}(\cdot) \in \Xi^{h}, \tau \in\left[t_{0}, \vartheta\right]$ и всякой пары $\left(x_{0}, \xi(\cdot)\right) \in X_{0} \times \Xi^{h}$, совместимой с тройкой $\left(\bar{x}_{0}, \bar{\xi}(\cdot), u_{\bar{x}_{0}, \bar{\xi}(\cdot)}(\cdot)\right)$ до момента $\tau$, при всех $t \in\left[t_{0}, \tau\right]$ выполняется $u_{x_{0}, \xi(\cdot)}(t)=u_{\bar{x}_{0}, \bar{\xi}(\cdot)}(t)$.

Тривиальный пример пакета программ произвольной точности $h$ - одноэлементное семейство $\left(u_{x_{0}, \xi(\cdot)}(\cdot)\right)_{x_{0} \in X_{0}, \xi(\cdot) \in \Xi^{h}}$, где $u_{x_{0}, \xi(\cdot)}(\cdot)$ суть одна и та же программа $u(\cdot)$ при всех $\left(x_{0}, \xi(\cdot)\right) \in X_{0} \times \Xi^{h}$. Другой пример - пакет программ точности $h$, порождаемый позиционной стратегией; он будет играть важную роль в наших последующих построениях (см. теорему 2.1). Дадим соответствующее определение. Выше было замечено, что для любой позиционной стратегии $S$, любого $x_{0} \in X_{0}$ и любого $\xi(\cdot) \in \Xi$ существует единственный управляемый процесс $\left(u_{x_{0}, \xi(\cdot)}(\cdot), x_{x_{0}, \xi(\cdot)}(\cdot), y_{x_{0}, \xi(\cdot)}(\cdot)\right)$ с начальным состоянием $x_{0}$ под действием стратегии $S$ при помехе наблюдения $\xi(\cdot)$; семейство $\left(u_{x_{0}, \xi(\cdot)}(\cdot)\right)_{x_{0} \in X_{0}, \xi(\cdot) \in \Xi^{h}}$ программ, получающееся варьированием начального состояния $x_{0} \in X_{0}$ и помехи наблюдения $\xi(\cdot) \in \Xi^{h}$, есть, как нетрудно установить, пакет программ точности $h$; этот пакет программ точности $h$ будем называть порожденным позиционной стратегией $S$.

Для пакетов программ рассмотрим задачу, аналогичную задаче позиционного наведения. Пакет программ $\left(u_{x_{0}, \xi(\cdot)}(\cdot)\right)_{x_{0} \in X_{0}, \xi(\cdot) \in \Xi^{h}}$ точности $h$ назовем $\varepsilon$-наводящим $(\varepsilon \geqslant 0)$, если для каждой пары $\left(\bar{x}_{0}, \bar{\xi}(\cdot)\right) \in X_{0} \times \Xi^{h}$ и каждой пары $\left(x_{0}, \xi(\cdot)\right) \in X_{0} \times \Xi^{h}$, совместимой с тройкой $\left(\bar{x}_{0}, \bar{\xi}(\cdot), u_{\bar{x}_{0}, \bar{\xi}(\cdot)}\right)$ до момента $\vartheta$, выполняется $x\left(\vartheta \mid x_{0}, u_{\bar{x}_{0}, \xi(\cdot)}(\cdot)\right) \in[M]^{\varepsilon}$. Скажем, что разрешима задача naкетного наведения, если для любого $\varepsilon>0$ найдется $\varepsilon$-наводящий пакет программ некоторой положительной точности $h$.

ПримеР 2.1. Рассмотрим ситуацию примера 1.1. Легко видеть, что при всяком $h<2$ семейство $\left(u_{x_{0}, \xi(\cdot)}(\cdot)\right)_{x_{0} \in X_{0}, \xi(\cdot) \in \Xi^{h}}$ такое, что $u_{-1, \xi(\cdot)}(t)=1$ и $u_{1, \xi(\cdot)}(t)=-1(t \in[0,1])$ для любого $\xi(\cdot) \in \Xi^{h}$, есть 0-наводящий пакет программ. Задача пакетного наведения разрешима.

ПримеР 2.2. Обратимся к ситуации из примера 1.2. Из приведенных в примере рассмотрений легко вывести, что при всяком $h<1$ семейство $\left(u_{x_{0}, \xi(\cdot)}(\cdot)\right)_{x_{0} \in X_{0}, \xi(\cdot) \in \Xi^{h},}$ где $u_{-1, \xi(\cdot)}(t)=0, \quad u_{-1, \xi(\cdot)}(t)=0 \quad(t \in[-3,0))$, $u_{-1, \xi(\cdot)}(t)=-1, \quad u_{-1, \xi(\cdot)}(t)=1 \quad(t \in[0,1])$ для любого $\xi(\cdot) \in \Xi^{h}$, есть 0 -наводящий пакет программ. Задача пакетного наведения разрешима. 
Итак, для приведенных примеров, при неразрешимости задачи программного наведения, имеем одновременную разрешимость задачи позиционного наведения и задачи пакетного наведения. Ниже мы покажем, что в общем случае задачи позиционного наведения и пакетного наведения эквивалентны: разрешимость (неразрешимость) одной из них влечет разрешимость (неразрешимость) другой.

ЗАмЕчание 2.1. Если пакет программ $\left(u_{x_{0}, \xi(\cdot)}(\cdot)\right)_{x_{0} \in X_{0}, \xi(\cdot) \in \Xi^{h}}$ точности $h$ является $\varepsilon$-наводящим, то для любого положительного $\eta<h$ суженный пакет программ $\left(u_{x_{0}, \xi(\cdot)}(\cdot)\right)_{x_{0} \in X_{0}, \xi(\cdot) \in \Xi^{\eta}}$ точности $\eta$ тоже $\varepsilon$-наводящий.

Первый факт, свидетельствующий о связи между задачами позиционного наведения и пакетного наведения, отражен в следующей теореме.

Теорема 2.1. Пусть задача пакетного наведения не разрешима. Тогда задача позиционного наведения также не разрешима.

ДокАзАтельство. Так как задача пакетного наведения не разрешима, то существует $\varepsilon>0$ такое, что никакой пакет программ $\left(u_{x_{0}, \xi(\cdot)}(\cdot)\right)_{x_{0} \in X_{0}, \xi(\cdot) \in \Xi^{h}}$ произвольной точности $h>0$ не является $\varepsilon$-наводящим. Последнее означает, что найдутся пара $\left(\bar{x}_{*}, \bar{\xi}_{*}(\cdot)\right) \in X_{0} \times \Xi^{h}$ и пара $\left(x_{*}, \xi_{*}(\cdot)\right) \in X_{0} \times \Xi^{h}$, совместимая с тройкой $\left(\bar{x}_{*}, \bar{\xi}_{*}(\cdot), u_{\bar{x}_{*}, \bar{\xi}(\cdot)}(\cdot)\right)$ до момента $\vartheta$, т.е. удовлетворяющая соотношению

$$
s\left(x\left(t \mid x_{*}, u_{\bar{x}_{*}, \bar{\xi}_{*}(\cdot)}(\cdot)\right)\right)+\xi_{*}(t)=s\left(x\left(t \mid \bar{x}_{*}, u_{\bar{x}_{*}, \bar{\xi}_{*}(\cdot)}(\cdot)\right)\right)+\bar{\xi}_{*}(t) \quad\left(t \in\left[t_{0}, \vartheta\right]\right),
$$

такие, что

$$
x\left(\vartheta \mid x_{*}, u_{\bar{x}_{*}, \bar{\xi}_{*}(\cdot)}(\cdot)\right) \notin[M]^{\varepsilon} .
$$

В пределах данного доказательства договоримся называть четверку $\left(\bar{x}_{*}, \bar{\xi}_{*}(\cdot)\right.$, $\left.x_{*}, \xi_{*}(\cdot)\right) \varepsilon$-отклоняющей для пакета программ $\left(u_{x_{0}, \xi(\cdot)}(\cdot)\right)_{x_{0} \in X_{0}, \xi(\cdot) \in \Xi^{h}}$.

Дальнейшее доказательство проведем от противного. Предположим, что задача позиционного наведения разрешима. Пусть $\left(S_{h}\right)_{h>0}$, где $S_{h}=\left(\tau_{i}^{h}, U_{i}^{h}\right)_{i=0}^{l^{h}}$ $(h>0),-$ наводящее семейство позиционных стратегий. Рассмотрим пакет программ $\left(u_{x_{0}, \xi(\cdot)}^{h}\right)_{x_{0} \in X_{0}, \xi(\cdot) \in \Xi^{h}}$ точности $h$, порожденный позиционной стратегией $S_{h}$. По определению этого пакета программ для каждых $x_{0} \in X_{0}$ и $\xi(\cdot) \in \Xi^{h}$ программа $u_{x_{0}, \xi(\cdot)}^{h}(\cdot)$ есть компонента (единственного) управляемого процесса $\left(u_{x_{0}, \xi(\cdot)}^{h}(\cdot), x_{x_{0}, \xi(\cdot)}^{h}(\cdot), y_{x_{0}, \xi(\cdot)}^{h}(\cdot)\right)$ с начальным состоянием $x_{0}$ под действием стратегии $S_{h}$ при помехе наблюдения $\xi(\cdot)$.

Зафиксируем произвольное $h>0$. Возьмем четверку $\left(\bar{x}_{*}, \bar{\xi}_{*}(\cdot), x_{*}, \bar{\xi}_{*}(\cdot)\right)$, $\varepsilon$-отклоняющую для пакета программ $\left(u_{x_{0}, \xi(\cdot)}^{h}\right)_{x_{0} \in X_{0}, \xi(\cdot) \in \Xi^{h}}$ точности $h$. Для этой четверки выполняются соотношения (см. (2.1), (2.2))

$$
\begin{gathered}
s\left(x\left(t \mid x_{*}, u_{\bar{x}_{*}, \bar{\xi}_{*}(\cdot)}^{h}(\cdot)\right)\right)+\xi_{*}(t)=s\left(x\left(t \mid \bar{x}_{*}, u_{\bar{x}_{*}, \bar{\xi}_{*}(\cdot)}^{h}(\cdot)\right)\right)+\bar{\xi}_{*}(t) \quad\left(t \in\left[t_{0}, \vartheta\right]\right), \\
x\left(\vartheta \mid x_{*}, u_{\bar{x}_{*}, \bar{\xi}_{*}(\cdot)}^{h}(\cdot)\right) \notin[M]^{\varepsilon}
\end{gathered}
$$


Полагая

$$
\begin{array}{ll}
x^{h}(\cdot)=x\left(t \mid x_{*}, u_{\bar{x}_{*}^{h}, \bar{\xi}(\cdot)}^{h}(\cdot)\right), & y^{h}(\cdot)=s\left(x^{h}(\cdot)\right)+\xi_{*}(\cdot), \\
\bar{x}^{h}(\cdot)=x\left(t \mid \bar{x}_{*}, u_{\bar{x}_{*}^{h}, \bar{\xi}(\cdot)}^{h}(\cdot)\right), & \bar{y}^{h}(\cdot)=s\left(\bar{x}^{h}(\cdot)\right)+\bar{\xi}_{*}(\cdot),
\end{array}
$$

перепишем (2.3), (2.4) в более компактной форме:

$$
\begin{gathered}
y^{h}(t)=\bar{y}^{h}(t) \quad\left(t \in\left[t_{0}, \vartheta\right]\right), \\
x^{h}(\vartheta) \notin[M]^{\varepsilon} .
\end{gathered}
$$

Покажем, что тройка $\left(u_{\bar{x}_{*}, \bar{\xi}_{*}(\cdot)}^{h}(\cdot), x^{h}(\cdot), \bar{y}^{h}(\cdot)\right)$ является управляемым процессом с начальным состоянием $x_{*}$ под действием позиционной стратегии $S_{h}$ при помехе наблюдения $\xi_{*}(\cdot)$. Так как $x^{h}(\cdot)$ - движение из начального состояния $x_{*}$ под действием программы $u_{\bar{x}_{*}, \bar{\xi}_{*}(\cdot)}^{h}(\cdot)$ (см. первое равенство в $\left.(2.5)\right)$ и при этом верно второе равенство из $(2.5)$, то достаточно установить, что при всех $i=0, \ldots, l^{h}$ справедливо

$$
u_{\bar{x}_{*}, \bar{\xi}_{*}(\cdot)}^{h}(t)=U_{i}^{h}\left(u_{\bar{x}_{*}, \bar{\xi}_{*}(\cdot), \tau_{i}}^{h}(\cdot), \bar{y}_{\tau_{i}}^{h}(\cdot)\right) \quad\left(t \in\left[\tau_{i}^{h}, \tau_{i+1}^{h}\right)\right),
$$

где $u_{\bar{x}_{*}, \bar{\xi}_{*}(\cdot), \tau_{i}}^{h}(\cdot)$ - сужение $u_{\bar{x}_{*}, \bar{\xi}_{*}(\cdot)}^{h}(\cdot)$ на $\left[t_{0}, \tau_{i}^{h}\right]$ и $\bar{y}_{\tau_{i}}^{h}(\cdot)$ - сужение $\bar{y}^{h}(\cdot)$ на $\left[t_{0}, \tau_{i}^{h}\right]$. Докажем соотношения (2.9). Рассмотрим управляемый процесс $\left(u_{\bar{x}_{*}, \bar{\xi}_{*}(\cdot)}^{h}(\cdot), x_{\bar{x}_{*}, \bar{\xi}_{*}(\cdot)}^{h}(\cdot), y_{\bar{x}_{*}, \bar{\xi}_{*}(\cdot)}^{h}(\cdot)\right)$ с начальным состоянием $\bar{x}_{*}$ под действием стратегии $S_{h}$ при помехе наблюдения $\bar{\xi}_{*}(\cdot)$. По определению этого управляемого процесса, имеем, во-первых,

$$
x_{\bar{x}_{*}, \bar{\xi}_{*}(\cdot)}^{h}(\cdot)=x\left(t \mid \bar{x}_{*}, u_{\bar{x}_{*}^{h}, \bar{\xi}_{*}(\cdot)}^{h}(\cdot)\right), \quad y_{\bar{x}_{*}, \bar{\xi}_{*}(\cdot)}^{h}(\cdot)=s\left(x_{\bar{x}_{*}, \bar{\xi}_{*}(\cdot)}^{h}(\cdot)\right)+\bar{\xi}_{*}(\cdot)
$$

или же, в обозначениях (2.6),

$$
x_{\bar{x}_{*}, \bar{\xi}_{*}(\cdot)}^{h}(\cdot)=\bar{x}^{h}(\cdot), \quad y_{\bar{x}_{*}, \bar{\xi}_{*}(\cdot)}^{h}(\cdot)=\bar{y}^{h}(\cdot),
$$

и, во-вторых, при всех $i=0, \ldots, l^{h}$

$$
u_{\bar{x}_{*}, \bar{\xi}_{*}(\cdot)}^{h}(t)=U_{i}^{h}\left(u_{\bar{x}_{*}, \bar{\xi}_{*}(\cdot), \tau_{i}}^{h}(\cdot), y_{\bar{x}_{*}, \bar{\xi}_{*}(\cdot), \tau_{i}}^{h}(\cdot)\right) \quad\left(t \in\left[\tau_{i}^{h}, \tau_{i+1}^{h}\right)\right),
$$

где $y_{\bar{x}_{*}, \bar{\xi}_{*}(\cdot), \tau_{i}}^{h}(\cdot)$ - сужение $y_{\bar{x}_{*}, \bar{\xi}_{*}(\cdot)}^{h}(\cdot)$ на $\left[t_{0}, \tau_{i}^{h}\right]$. Последнее, в силу второго равенства в $(2.10)$, равносильно $(2.9)$. Итак, $\left(u_{\bar{x}_{*}^{h}, \bar{\xi}_{*}(\cdot)}^{h}(\cdot), x^{h}(\cdot), \bar{y}^{h}(\cdot)\right)$ есть управляемый процесс с начальным состоянием $x_{*}$ под действием позиционной стратегии $S_{h}$ при помехе наблюдения $\xi_{*}(\cdot) \in \Xi^{h}$. Так как семейство $\left(S_{h}\right)_{h>0}$ наводящее, то при достаточно малом $h$ выполняется $x^{h}(\vartheta) \in[M]^{\varepsilon}$. Это, однако, противоречит (2.8). Противоречие завершает доказательство.

В дальнейшем (точнее, при доказательстве теоремы 5.1) будем использовать понятие монотонно наводящего семейства пакетов программ.

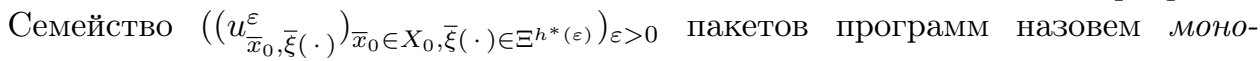
тонно наводяшим, если, во-первых, для каждого $\varepsilon>0$ пакет программ

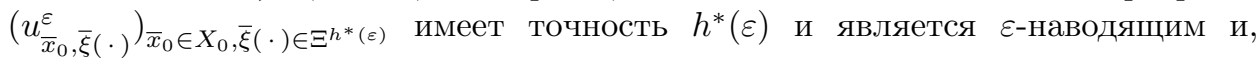
во-вторых, функция $\varepsilon \mapsto h^{*}(\varepsilon)>0$ монотонно возрастает. 
Лемма 2.1. Пусть разрешима задача пакетного наведения. Тогда существует монотонно наводящее семейство пакетов программ.

ДокАзАТЕЛЬство. Так как разрешима задача пакетного наведения, то для

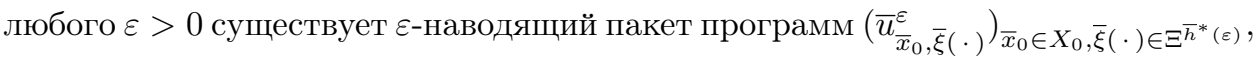
имеющий точность $\bar{h}^{*}(\varepsilon)$. Для $\varepsilon \geqslant 1 / 2$ примем $h^{*}(\varepsilon)=\bar{h}^{*}(1)$ и возьмем в ка-

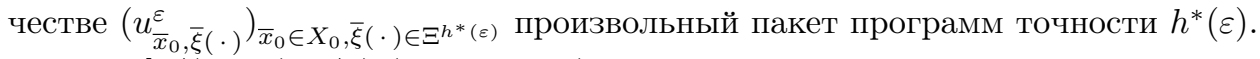
Для $\varepsilon \in[1 /(k+1), 1 / k)(k=1,2, \ldots)$ положим

$$
\begin{gathered}
h^{*}(\varepsilon)=\min \left\{\bar{h}^{*}(1 / j): j=1, \ldots, k+1\right\} \\
\left(u_{\bar{x}_{0}, \bar{\xi}(\cdot)}^{\varepsilon}\right)_{\bar{x}_{0} \in X_{0}, \bar{\xi}(\cdot) \in \Xi^{h^{*}(\varepsilon)}}=\left(\bar{u}_{\bar{x}_{0}, \bar{\xi}(\cdot)}^{1 /(k+1)}\right)_{\bar{x}_{0} \in X_{0}, \bar{\xi}(\cdot) \in \Xi^{h^{*}(1 /(k+1))}} .
\end{gathered}
$$

Тогда для $\varepsilon \in[1 /(k+1), 1 / k)$ пакет программ $\left(u_{\bar{x}_{0}, \bar{\xi}(\cdot)}\right)_{\bar{x}_{0} \in X_{0}, \bar{\xi}(\cdot) \in \Xi^{h^{*}(\varepsilon)}}$ является $(1 /(k+1))$-наводящим и, следовательно, $\varepsilon$-наводящим. Так как функция $\varepsilon \mapsto$ $h^{*}(\varepsilon)$ возрастает, то семейство $\left(\left(u_{\bar{x}_{0}, \bar{\xi}(\cdot)}\right)_{\bar{x}_{0} \in X_{0}, \bar{\xi}(\cdot) \in \Xi^{h^{*}(\varepsilon)}}\right)_{\varepsilon>0}$ пакетов программ является монотонно наводящим. Доказательство закончено.

\section{3. Стабильные мосты}

Теперь наша цель - обратить теорему 2.1. Таким образом, в предположении о разрешимости задачи пакетного наведения нам надлежит построить разрешающее семейство позиционных стратегий. Эту программу мы реализуем в следующих разделах. В данном разделе мы введем первую часть конструкции предлагаемого решения - стабильные мосты. В основе конструкции - метод экстремального сдвига Н.Н. Красовского из теории позиционных дифференциальных игр [6].

В теории позиционных дифференциальных игр метод экстремального сдвига объединяет два ключевых элемента - стабильный мост и связанную с ним позиционную стратегию экстремального сдвига. Стабильный мост - это некоторое семейство множеств в фазовом пространстве конфликтно-управляемой системы, параметризованное переменной времени. Элемент семейства, отвечающий моменту времени $\tau$, обычно называют сечением моста в этот момент времени. В контексте дифференциально-игровой задачи позиционного наведения, аналогичной рассматриваемой здесь (но с полной фазовой информацией), стабильный мост можно интерпретировать как “дорогу", соединяющую начальное состояние системы (в том смысле, что начальное состояние системы содержится в начальном сечении моста) и целевое множество системы (в том смысле, что последнее по времени сечение моста лежит в целевом множестве). Связанная со стабильным мостом позиционная стратегия экстремального сдвига - это способ управления "игрока-союзника", позволяющий ему удерживать систему на “дороге" (вблизи “дороги") в течение всего времени управления независимо от реализации фактора неопределенности (программы "игрока-противника"); в итоге, в последний момент времени система гарантированно приводится на целевое множество. Свойство стабильности моста есть критерий существования способа управления "игрока-союзника", гарантированно удерживающего систему на "дороге". 
В нашем случае аналогом сечения стабильного моста в момент времени $\tau$ служит специальное множество - обозначим его $W(\tau)$ - подмножеств из пространства состояний управляемой системы. Множество $W(\tau)$ связано с некото-

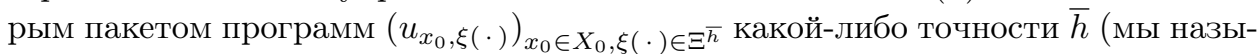
ваем $W(\tau)$ фазовым образом данного пакета программ в момент $\tau)$. Каждый элемент из $W(\tau)$ есть множество - обозначим его здесь $X^{\eta}(\tau)$ - в пространстве состояний системы, определяемое одной из программ, $\bar{u}(\cdot)=u_{\bar{x}_{0}, \bar{\xi}}(\cdot)$, данного пакета; точнее, $X^{\eta}(\tau)$ - это совокупность состояний, совместимых в момент $\tau$ с сигналом $y(t)=s(\bar{x}(t))+\bar{\xi}(t)$, наблюдаемым вдоль движения $\bar{x}(\cdot)=x\left(\cdot \mid \bar{x}_{0}, \bar{u}(\cdot)\right)$ на отрезке времени $\left[t_{0}, \tau\right]$ при некоторой помехе $\bar{\xi}(\cdot)$ точности $\eta \leqslant \bar{h}$. Такое определение, в частности, подразумевает, что при любом наблюдении точности $\eta$, произведенном в начальный момент $t_{0}$, каждое “потенциально истинное", т.е. совместимое с этим наблюдением, начальное состояние попадает в одно из множеств $X^{\eta}\left(t_{0}\right) \in W\left(t_{0}\right)$ (см. ниже лемму 3.2 ); это свойство служит естественным аналогом упомянутого выше свойства начального сечения стабильного моста, применямого в теории позиционных дифференциальных игр. Другое достаточно понятное следствие из определения множеств

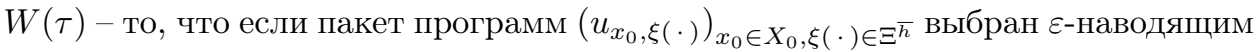
(что мы и будем предполагать, поскольку будем исходить из того, что задача пакетного наведения разрешима), то каждое множество $X^{\eta}(\vartheta) \in W(\vartheta)$ содержится в $\varepsilon$-окрестности целевого множества $M$ (см. лемму 3.3 ): получаем аналог стандартного свойства конечного сечения стабильного моста. Наконец, оказывается, что для множеств $W(\tau)$ имеет место также и свойство, аналогичное свойству стабильности моста, используемого в теории позиционных дифференциальных игр (лемма 3.4).

Ввиду указанных аналогий семейство множеств $W(\tau)$, параметризованное временем $\tau \in\left[t_{0}, \vartheta\right]$, мы также называем мостом. Отметим, что параметр $\eta$, присутствующий в определении множеств $W(\tau)$, мы полагаем, вообще говоря, зависящим от $\tau: \eta=\eta(\tau)$; точнее, считаем, что $\eta(\tau)$ не убывает при росте $\tau$ и (как упомянуто выше) не превосходит точности $\bar{h}$ "базового" паке-

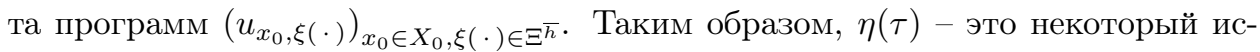
кусственный переменный (“ухудшающийся") параметр точности наблюдения, “лучший", чем параметр $\bar{h}$ "базового" пакета программ. Такое усложнение в определении (естественнее всего было бы положить $\eta(\tau)=\bar{h}$ ) есть важная техническая "заготовка" для построения работоспособной позиционной стратегии, аналогичной стратегии экстремального сдвига из теории позиционных дифференциальных игр (см. начало настоящего неформального комментария), т.е. в данном случае такой позиционной стратегии, которая удерживает множество всех текущих "потенциально истинных" состояний системы вблизи текущих сечений моста. В следующих разделах при построении такой позиционной стратегии $\eta(\tau)$ подбирается специальным образом и служит верхней (“ухудшенной”) оценкой для истинной точности $h$ наблюдения (см. условие (iii) в лемме 5.3).

Перейдем к строгому изложению, оставляя место для кратких неформальных комментариев. Сначала определим прототипы элементов $X^{\eta}(\tau) \in W(\tau)$, являющиеся, как сказано выше, множествами в пространстве состояний. Для всяких $\eta \geqslant 0, \tau \in\left[t_{0}, \vartheta\right]$ и тройки $\left(\bar{x}_{0}, \bar{\xi}(\cdot), \bar{u}(\cdot)\right) \in X_{0} \times \Xi \times \mathscr{U}$ через $X_{0}^{\eta}\left(\tau \mid \bar{x}_{0}, \bar{\xi}(\cdot), \bar{u}(\cdot)\right)$ будем обозначать множество всех $x_{0} \in X_{0}$, для каждого 
из которых найдется $\xi(\cdot) \in \Xi^{\eta}$ такое, что пара $\left(x_{0}, \xi(\cdot)\right)$ совместима с тройкой $\left(\bar{x}_{0}, \bar{\xi}(\cdot), \bar{u}(\cdot)\right)$ до момента $\tau$; данное множество назовем множеством начальных состояний, $\eta$-совместимых с тройкой $\left(\bar{x}_{0}, \bar{\xi}(\cdot), \bar{u}(\cdot)\right)$ до момента $\tau$. Понятно, что все начальные состояния из данного множества, и только они, "потенциально истинны" на момент $\tau$, коль скоро на отрезке времени $t$, предшествующем $\tau$, применялась программа $\bar{u}(\cdot)$ и наблюдался сигнал $y(t)=s\left(x\left(t \mid \bar{x}_{0}, \bar{u}(\cdot)\right)\right)+\bar{\xi}(t)$. Также положим

$$
X^{\eta}\left(\tau \mid \bar{x}_{0}, \bar{\xi}(\cdot), \bar{u}(\cdot)\right)=\left\{x\left(\tau \mid x_{0}, \bar{u}(\cdot)\right): x_{0} \in X_{0}^{\eta}\left(\tau \mid \bar{x}_{0}, \bar{\xi}(\cdot), \bar{u}(\cdot)\right)\right\}
$$

последнее множество назовем множеством состояний, $\eta$ "совместимых с тройкой $\left(\bar{x}_{0}, \bar{\xi}(\cdot), \bar{u}(\cdot)\right)$ в момент $\tau$. Все состояния из данного множества, и только они, суть "потенциально истинные" состояния системы в текущий момент $\tau$ при условии, что до момента $\tau$ применялась программа $\bar{u}(\cdot)$ и при всех $t \leqslant \tau$ наблюдется вышеуказанный сигнал $y(t)$. Заметим, что множество (3.1) заведомо содержит точку $x\left(\tau \mid \bar{x}_{0}, \bar{u}(\cdot)\right)$ и, таким образом, непусто. Заметим также, что при $\tau=t_{0}$ для всех $\bar{x}_{0} \in X_{0}, \bar{\xi}(\cdot) \in \Xi, \eta \geqslant 0$ имеем:

$$
\begin{aligned}
X^{\eta}\left(t_{0} \mid \bar{x}_{0}, \bar{\xi}(\cdot), \bar{u}(\cdot)\right) & =X_{0}^{\eta}\left(t_{0} \mid \bar{x}_{0}, \bar{\xi}(\cdot), \bar{u}(\cdot)\right) \\
& =\left\{x_{0} \in X_{0}:\left|s\left(x_{0}\right)-s\left(\bar{x}_{0}\right)-\bar{\xi}\left(t_{0}\right)\right| \leqslant \eta\right\} .
\end{aligned}
$$

Лемма 3.1. Пусть $\eta \geqslant 0, \bar{x}_{0}, x_{*} \in X_{0}, \bar{\xi}(\cdot), \xi_{*}(\cdot) \in \Xi^{\eta}, \bar{u}(\cdot) \in \mathscr{U}$ u napa $\left(x_{*}, \xi_{*}(\cdot)\right)$ совместима с тройкой $\left(\bar{x}_{0}, \bar{\xi}(\cdot), \bar{u}(\cdot)\right)$ до момента $\tau \in\left[t_{0}, \vartheta\right]$. Тогда справедливы равенства

$$
\begin{aligned}
& X_{0}^{\eta}\left(\tau \mid x_{*}, \xi_{*}(\cdot), \bar{u}(\cdot)\right)=X_{0}^{\eta}\left(\tau \mid \bar{x}_{0}, \bar{\xi}(\cdot), \bar{u}(\cdot)\right), \\
& X^{\eta}\left(\tau \mid x_{*}, \xi_{*}(\cdot), \bar{u}(\cdot)\right)=X^{\eta}\left(\tau \mid \bar{x}_{0}, \bar{\xi}(\cdot), \bar{u}(\cdot)\right) .
\end{aligned}
$$

ДоказАтельство. Докажем (3.3). Возьмем произвольный элемент $x_{0} \in$ $X_{0}^{\eta}\left(\tau \mid x_{*}, \xi_{*}(\cdot), \bar{u}(\cdot)\right)$. По определению последнего множества существует $\xi(\cdot) \in \Xi^{\eta}$ такое, что пара $\left(x_{0}, \xi(\cdot)\right)$ совместима с тройкой $\left(x_{*}, \xi_{*}(\cdot), \bar{u}(\cdot)\right)$ до момента $\tau$, т.е.

$$
s\left(x\left(t \mid x_{0}, \bar{u}(\cdot)\right)\right)+\xi(t)=s\left(x\left(t \mid x_{*}, \bar{u}(\cdot)\right)\right)+\xi_{*}(t) \quad\left(t \in\left[t_{0}, \tau\right]\right) .
$$

Но по условию пара $\left(x_{*}, \xi_{*}(\cdot)\right)$ совместима с тройкой $\left(\bar{x}_{0}, \bar{\xi}(\cdot), \bar{u}(\cdot)\right)$ до момента $\tau$, т.е.

$$
s\left(x\left(t \mid x_{*}, \bar{u}(\cdot)\right)\right)+\xi_{*}(t)=s\left(x\left(t \mid \bar{x}_{0}, \bar{u}(\cdot)\right)\right)+\bar{\xi}(t) \quad\left(t \in\left[t_{0}, \tau\right]\right) .
$$

Отсюда и из (3.5)

$$
s\left(x\left(t \mid x_{0}, \bar{u}(\cdot)\right)\right)+\xi(t)=s\left(x\left(t \mid \bar{x}_{0}, \bar{u}(\cdot)\right)\right)+\bar{\xi}(t) \quad\left(t \in\left[t_{0}, \tau\right]\right),
$$

что означает, что пара $\left(x_{0}, \xi(\cdot)\right)$ совместима с тройкой $\left(\bar{x}_{0}, \bar{\xi}(\cdot), \bar{u}(\cdot)\right)$ до момента $\tau$. Значит, $x_{0} \in X_{0}^{\eta}\left(\tau \mid \bar{x}_{0}, \bar{\xi}(\cdot), \bar{u}(\cdot)\right)$. Из-за произвольности $x_{0} \in$ $X^{\eta}\left(\tau \mid x_{*}, \xi_{*}(\cdot), \bar{u}(\cdot)\right)$ заключаем, что

$$
X_{0}^{\eta}\left(\tau \mid x_{*}, \xi_{*}(\cdot), \bar{u}(\cdot)\right) \subset X_{0}^{\eta}\left(\tau \mid \bar{x}_{0}, \bar{\xi}(\cdot), \bar{u}(\cdot)\right) .
$$


Далее, так как пара $\left(x_{*}, \xi_{*}(\cdot)\right)$ совместима с тройкой $\left(\bar{x}_{0}, \bar{\xi}(\cdot), \bar{u}(\cdot)\right)$ до момента $\tau$, то пара $\left(\bar{x}_{0}, \bar{\xi}(\cdot)\right)$ совместима с тройкой $\left(x_{*}, \xi_{*}(\cdot), \bar{u}(\cdot)\right)$. Поэтому, меняя местами в предыдущем рассуждении пары $\left(x_{*}, \xi_{*}(\cdot)\right)$ и $\left(\bar{x}_{0}, \bar{\xi}(\cdot)\right)$, приходим к вложению, противоположному (3.6), что, вместе с (3.6), доказывает равенство (3.3). Наконец, из определения множеств, записанных в левой и правой частях (3.4) (см. (3.1)), и из (3.3) следует справедливость равенства (3.4). Лемма 3.1 доказана.

СлеДСТвиЕ 3.1. Пусть $\eta \geqslant 0,\left(u_{x_{0}}\right)_{x_{0} \in X_{0}, \xi(\cdot) \in \Xi^{\bar{h}}}-$ nакет программ точности $\bar{h} \geqslant \eta, \bar{x}_{0}, x_{*} \in X_{0}, \bar{\xi}(\cdot), \xi_{*}(\cdot) \in \Xi^{\eta}$ u nара $\left(x_{*}, \xi_{*}(\cdot)\right)$ совместима с тройкой $\left(\bar{x}_{0}, \bar{\xi}(\cdot), u_{\bar{x}_{0}, \bar{\xi}(\cdot)}(\cdot)\right)$ до момента $\tau \in\left[t_{0}, \vartheta\right]$. Тогда справедливы равенства

$$
\begin{aligned}
& X_{0}^{\eta}\left(\tau \mid x_{*}, \xi_{*}(\cdot), u_{x_{*}, \xi_{*}(\cdot)}(\cdot)\right)=X_{0}^{\eta}\left(\tau \mid \bar{x}_{0}, \bar{\xi}(\cdot), u_{\bar{x}_{0}, \bar{\xi}(\cdot)}(\cdot)\right), \\
& X^{\eta}\left(\tau \mid x_{*}, \xi_{*}(\cdot), u_{x_{*}, \xi_{*}(\cdot)}(\cdot)\right)=X^{\eta}\left(\tau \mid \bar{x}_{0}, \bar{\xi}(\cdot), u_{\bar{x}_{0}, \bar{\xi}(\cdot)}(\cdot)\right) .
\end{aligned}
$$

ДокАЗАТЕЛЬСтво. По лемме 3.1

$$
\begin{aligned}
& X_{0}^{\eta}\left(\tau \mid x_{*}, \xi_{*}(\cdot), u_{\bar{x}_{0}, \bar{\xi}(\cdot)}(\cdot)\right)=X_{0}^{\eta}\left(\tau \mid \bar{x}_{0}, \bar{\xi}(\cdot), u_{\bar{x}_{0}, \bar{\xi}(\cdot)}(\cdot)\right), \\
& X^{\eta}\left(\tau \mid x_{*}, \xi_{*}(\cdot), u_{\bar{x}_{0}, \bar{\xi}(\cdot)}(\cdot)\right)=X^{\eta}\left(\tau \mid \bar{x}_{0}, \bar{\xi}(\cdot), u_{\bar{x}_{0}, \bar{\xi}(\cdot)}(\cdot)\right) .
\end{aligned}
$$

Так как по определяющему свойству пакета программ $u_{x_{*}, \xi_{*}(\cdot)}(t)=u_{\bar{x}_{0}, \bar{\xi}(\cdot)}(t)$ при всех $t \in\left[t_{0}, \tau\right]$, то имеем (3.7) и (3.8). Следствие 3.1 доказано.

Переходим к определению моста как семейства классов $W(\tau)$. Для каждых

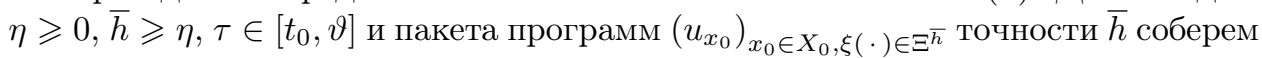
в один класс все множества состояний, $\eta$-совместимых в момент $\tau$ с тройками $\left(\bar{x}_{0}, \bar{\xi}(\cdot), \bar{u}_{\bar{x}_{0}, \bar{\xi}(\cdot)}(\cdot)\right) ;$ именно, класс множеств

$$
W(\tau)=\left\{X^{\eta}\left(\tau \mid \bar{x}_{0}, \bar{\xi}(\cdot), u_{\bar{x}_{0}, \bar{\xi}(\cdot)}(\cdot)\right):\left(\bar{x}_{0}, \bar{\xi}(\cdot)\right) \in X_{0} \times \Xi^{\eta}\right\}
$$

будем называть фазовым $\eta$-образом в момент $\tau$ пакета программ $\left(u_{x_{0}, \xi(\cdot)}\right)_{x_{0} \in X_{0}, \xi(\cdot) \in \Xi^{\bar{h}}}$ точности $\bar{h}$.

Из этого определения, с учетом (3.2) сразу получаем, что при любом наблюдении точности $\eta$, произведенном в начальный момент $t_{0}$, соответствующее множество всех "потенциально истинных" начальных состояний есть элемент начального фазового образа $W\left(t_{0}\right)$ :

Лемма 3.2. Пусть $W\left(t_{0}\right)$ - фазовый $\eta$-образ в момент $t_{0}$ пакета программ точности $\bar{h} \geqslant \eta$ и $y\left(t_{0}\right)=s\left(\bar{x}_{0}\right)-\bar{\xi}\left(t_{0}\right)$ при некоторых $\bar{x}_{0} \in X_{0}$ u $\bar{\xi}(\cdot) \in \Xi^{\eta}$. Тогда $\left\{x_{0} \in X_{0}:\left|s\left(x_{0}\right)-y\left(t_{0}\right)\right| \leqslant \eta\right\} \in W\left(t_{0}\right)$.

Ввиду определения $\varepsilon$-наводящего пакета программ точности $\bar{h}$, очевидно следующее свойство конечного фазового образа $W(\vartheta)$.

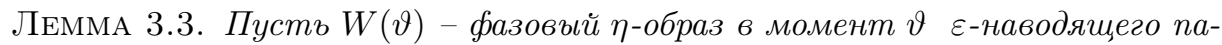
кета программ точности $\bar{h} \geqslant \eta$. Тогда для каждого $X(\vartheta) \in W(\vartheta)$ справедливо вложение $X(\vartheta) \subset[M]^{\varepsilon}$. 
Следующее определение - основное в настоящем разделе. Если $\eta(\cdot)-$ неотрицательная функция на $\left[t_{0}, \vartheta\right]$, то $\eta(\cdot)$-мостом, порожденным пакетом программ $\left(u_{x_{0}, \xi(\cdot)}\right)_{x_{0} \in X_{0}, \xi(\cdot) \in \Xi^{\bar{h}}}$ точности $\bar{h}$, будем называть семейство $(W(\tau))_{\tau \in\left[t_{0}, \vartheta\right]}$ такое, что для каждого $\tau \in\left[t_{0}, \vartheta\right]$ класс множеств $W(\tau)$ есть фазовый $\eta(\tau)$-образ в момент $\tau$ данного пакета программ:

$$
W(\tau)=\left\{X^{\eta(\tau)}\left(\tau \mid \bar{x}_{0}, \bar{\xi}(\cdot), u_{\bar{x}_{0}, \bar{\xi}(\cdot)}(\cdot)\right):\left(\bar{x}_{0}, \bar{\xi}(\cdot)\right) \in X_{0} \times \Xi^{\eta(\tau)}\right\} .
$$

Последнее семейство - аналог стабильного моста из теории позиционных дифференциальных игр. Свойство, аналогичное свойству стабильности, выражено в следующей лемме.

ЛЕмма 3.4. Пусть $\eta(\cdot)$ - неотрицательная неубывающая функиия на $\left[t_{0}, \vartheta\right], \bar{h} \geqslant \max _{\tau \in\left[t_{0}, \vartheta\right]} \eta(\tau) u(W(\tau))_{\tau \in\left[t_{0}, \vartheta\right]}-\eta(\cdot)$-мост, порожденньй какимлибо пакетом программ точности $\bar{h}$. Тогда для любых $t_{1}, t_{2} \in\left[t_{0}, \vartheta\right]$, где $t_{2} \geqslant t_{1}$, любого $X\left(t_{1}\right) \in W\left(t_{1}\right)$ и любого $x_{1} \in X\left(t_{1}\right)$ существуют $X\left(t_{2}\right) \in W\left(t_{2}\right)$ u программа $u(\cdot)$ такие, что решение $x(\cdot)$ на $\left[t_{0}, \vartheta\right]$ дифференииального уравнения $\dot{x}(t)=f(t, x(t), u(t))$ (см. (1.1)), задаваемое условием $x\left(t_{1}\right)=x_{1}$, единственно и удовлетворяет включению $x\left(t_{2}\right) \in X\left(t_{2}\right)$.

ДокаЗАТЕЛЬСтво. Пусть $\eta(\cdot)$-мост $(W(\tau))_{\tau \in\left[t_{0}, \vartheta\right]}$ порожден пакетом программ $\left(u_{x_{0}, \xi(\cdot)}\right)_{x_{0} \in X_{0}, \xi(\cdot) \in \Xi^{\bar{h}}}$ точности $\bar{h}$. Поскольку $X\left(t_{1}\right) \in W\left(t_{1}\right)$, то $X\left(t_{1}\right)=$ $X^{\eta\left(t_{1}\right)}\left(t_{1} \mid \bar{x}_{0}, \bar{\xi}(\cdot), u_{\bar{x}_{0}, \bar{\xi}(\cdot)}(\cdot)\right)$ для некоторой пары $\left(\bar{x}_{0}, \bar{\xi}(\cdot)\right) \in X_{0} \times \Xi^{\eta\left(t_{1}\right)}$. Так как $x_{1} \in X\left(t_{1}\right)$, то

$$
x_{1}=x\left(\cdot \mid x_{0}, u_{\bar{x}_{0}, \bar{\xi}(\cdot)}(\cdot)\right),
$$

где $x_{0} \in X_{0}^{\eta\left(t_{1}\right)}\left(t_{1} \mid \bar{x}_{0}, \bar{\xi}(\cdot), u_{\bar{x}_{0}, \bar{\xi}(\cdot)}(\cdot)\right)$.

Последнее означает, что существует $\xi(\cdot) \in \Xi^{\eta\left(t_{1}\right)}$ такое, что пара $\left(x_{0}, \xi(\cdot)\right)$ совместима с тройкой $\left(\bar{x}_{0}, \bar{\xi}(\cdot), u_{\bar{x}_{0}, \bar{\xi}(\cdot)}(\cdot)\right)$ до момента $t_{1}$. Тогда по определяющему свойству пакета программ $u_{x_{0}, \xi(\cdot)}(t)=u_{\bar{x}_{0}, \bar{\xi}(\cdot)}(t)\left(t \in\left[t_{0}, \tau\right]\right)$. Отсюда и из (3.11), вводя движение $x_{1}(\cdot)=x\left(\cdot \mid x_{0}, u_{x_{0}, \xi(\cdot)}(\cdot)\right)$, заключаем, что $x_{1}=x\left(t_{1}\right)$. Ясно, что $x_{1}(\cdot)$ - единственное решение $x(\cdot)$ на $\left[t_{0}, \vartheta\right]$ дифференциального уравнения (1.1), удовлетворяющее условию $x\left(t_{1}\right)=x_{1}$. Осталось показать, что $x\left(t_{2}\right) \in X\left(t_{2}\right)$ для некоторого $X\left(t_{2}\right) \in W\left(t_{2}\right)$. Так как $\eta(\cdot)$ не убывает и $\xi(\cdot) \in \Xi^{\eta\left(t_{1}\right)}$, то $\xi(\cdot) \in \Xi^{\eta\left(t_{2}\right)}$. Kроме того, пара $\left(x_{0}, \xi(\cdot)\right)$, очевидно, совместима с тройкой $\left(x_{0}, \xi(\cdot), u_{x_{0}, \xi(\cdot)}(\cdot)\right)$ до момента $t_{2}$, откуда $x_{0} \in X_{0}^{\eta\left(t_{2}\right)}\left(t_{2} \mid x_{0}, \xi(\cdot), u_{x_{0}, \xi(\cdot)}(\cdot)\right)$. Поэтому $x\left(t_{2}\right) \in X\left(t_{2}\right)=X^{\eta\left(t_{2}\right)}\left(t_{2} \mid x_{0}, \xi(\cdot)\right.$, $\left.u_{x_{0}, \xi(\cdot)}(\cdot)\right)$. Ясно, что $X\left(t_{2}\right) \in W\left(t_{2}\right)$. Лемма 3.4 доказана.

Заключим данный раздел неформальным комментарием. В предположении о разрешимости задачи пакетного наведения свойство $\eta(\cdot)$-моста, отраженное в лемме 3.4 (назовем его здесь, следуя аналогу из теории позиционных дифференциальных игр, свойством стабильности), вместе со свойствами его начального и конечного сечений (леммы 3.2 и 3.3 ) дают возможность управляющей стороне успешно (сколь угодно точно) решить задачу наведения в некоторой искусственной схеме принятия решений с "игроком-противником". Эта схема предполагает, что управляющая сторона в каждый из моментов корректировки $\tau_{i}\left(\tau_{0}=t_{0}<\cdots<\tau_{l}<\vartheta\right)$ вырабатывает программу управления $u_{i}(\cdot)$ для применения на участке времени $\left[\tau_{i}, \tau_{i+1}\right)$. Априорные действия управляющей 
стороны таковы. Зная точность $h$ будущих наблюдений, управляющая сторона выбирает $\varepsilon$-наводящий пакет программ точности $h$ (при $h$ достаточно малом можно обеспечить сколь угодную малость $\varepsilon$ - это следует из разрешимости задачи пакетного наведения) и строит порожденный выбранным пакетом программ $\eta(\cdot)$-мост $(W(\tau))_{\tau \in\left[t_{0}, \vartheta\right]}$ при $\eta(\tau)=h$. В начальный момент корректировки $\tau_{0}=t_{0}$ управляющая сторона, произведя наблюдение $y\left(t_{0}\right)$ (точности $h$ ), строит множество $X\left(\tau_{0}\right)$ всех начальных состояний $x_{0}$, совместимых с этим наблюдением - таких, что $\left|s\left(x_{0}\right)-y\left(t_{0}\right)\right| \leqslant h$. По лемме $3.2 X\left(\tau_{0}\right) \in W\left(\tau_{0}\right)$. Далее вступает в действие "игрок-противник", который произвольным образом выбирает в пределах множества $X\left(\tau_{0}\right)$ точку $\bar{x}\left(\tau_{0}\right)$ - некий “эрзац" истинной начальной точки. "Игрок-противник" сообщает о своем выборе управляющей стороне. Зная выбор "игрока-противника", управляющая сторона обращается к свойству стабильности моста $(W(\tau))_{\tau \in\left[t_{0}, \vartheta\right]}($ лемма 3.4$)$. Руководствуясь этим свойством, управляющая сторона для применения на участке времени $\left[t_{0}, \tau_{1}\right)$ выбирает ту программу $u_{0}(\cdot)$, которая переводит (в силу уравнения системы) точку $\bar{x}\left(\tau_{0}\right) \in X\left(\tau_{0}\right)$ (в момент $\tau_{0}$ ) в точку $x\left(\tau_{1}\right)$ (в момент $\tau_{1}$ ), лежащую в некотором множестве $X\left(\tau_{1}\right) \in W\left(\tau_{1}\right)$. На следующем шаге действия аналогичны. "Игрок-противник" произвольным образом выбирает точку $\bar{x}\left(\tau_{1}\right) \in X\left(\tau_{1}\right)$ и сообщает о своем выборе управляющей стороне. Управляющая сторона, по свойству стабильности моста $(W(\tau))_{\tau \in\left[t_{0}, \vartheta\right]}$, выбирает для применения на участке времени $\left[\tau_{1}, \tau_{2}\right)$ программу $u_{1}(\cdot)$, переводящую точку $\bar{x}\left(\tau_{1}\right) \in X\left(\tau_{1}\right)$ в точку $x\left(\tau_{2}\right)$ из некоторого $X\left(\tau_{2}\right) \in W\left(\tau_{2}\right)$. Повторение такой "игры" на всех временнь́х участках $\left[\tau_{i}, \tau_{i+1}\right)$ генерирует последовательность множеств $X\left(\tau_{i}\right) \in W\left(\tau_{i}\right)$, последовательность "псевдореальных" точек $\bar{x}\left(\tau_{0}\right) \in X\left(\tau_{i}\right)$, выбираемых "игроком-противником", и последовательность точек $x\left(\tau_{i}\right) \in X\left(\tau_{i}\right)$, "проектируемых" управляющей стороной с помощью свойства стабильности. Свойство конечного сечения $W(\vartheta)$ моста (лемма 3.3 ) гарантирует, что в конечный момент времени "псевдореальная" точка попадает в $\varepsilon$-окрестность целевого множества: $\bar{x}(\vartheta) \in M^{\varepsilon}$ - в таком смысле можно говорить о разрешимости задачи наведения.

Обозначенная схема управления, разумеется, весьма далека от позиционной и не отвечает содержанию задачи - гарантированному приведению к целевому множеству реального состояния управляемой системы. С другой стороны, такая схема позволяет, на содержательном уровне, прояснить, что же непосредственно дает нам свойство стабильности мостов с точки зрения принятия решений. Мы привели этот неформальный комментарий для того, чтобы наглядно показать, что переход от свойств мостов, порожденных пакетами программ, к утверждению о разрешимости задачи позиционного наведения (при разрешимости задачи пакетного наведения) требует - так же, как и в теории позиционых дифференциальных игр - определенной дополнительной конструкции. Такой дополнительной конструкцией служит метод экстремального сдвига, описываемый и применяемый в следующих разделах.

\section{4. Информационные множества. Экстремальный сдвиг}

В этом разделе мы сначала введем в рассмотрение информационные множества - множества текущих позиций, совместимых с результатами прошлых на- 
блюдений (см. [30]); информационные множества заведомо содержат реальные состояния системы и аккумулируют всю информацию, необходимую управляющей стороне для принятия текущего решения об управлении. Затем мы покажем, как управляющей стороне следует выбирать свое управление в фиксированный момент корректировки $\tau=\tau_{i}$, с тем чтобы до следующего момента корректировки $\tau+\delta=\tau_{i+1}$ обеспечить удержание инфрмационного множества вблизи стабильного моста, порожденного выбранным пакетом программ. Предлагаемый метод выбора управления мы называем методом экстремального сдвига: он является прямой модификацией одноименного метода из теории позиционных дифференциальных игр. Выбор текущего управления по методу экстремального сдвига - основа позиционной стратегии экстремального сдвига, ведущей к решению задачи позиционного наведения при разрешимости задачи пакетного наведения (тема следующего раздела).

Перейдем к определениям. Если $x_{0} \in X_{0}$ и $u_{\tau}(\cdot)$ - программа до момента $\tau \in$ $\left[t_{0}, \vartheta\right]$, то через $x\left(t \mid x_{0}, u_{\tau}(\cdot)\right)$ будем обозначать движение до момента $\tau$ из $x_{0}$ под действием $u_{\tau}(\cdot)$, т.е. решение (Каратеодори) $x(\cdot)$ на $\left[t_{0}, \tau\right]$ уравнения $(1.1)$ с $u(t)=u_{\tau}(t)\left(t \in\left[t_{0}, \tau\right]\right)$ и начальным условием $x\left(t_{0}\right)=x_{0}$; заметим, что в силу условий $(\mathrm{A} 2)$ и (А3) движение $x\left(t \mid x_{0}, u_{\tau}(\cdot)\right)$ существует и единственно. Для всяких $\tau \in\left[t_{0}, \vartheta\right]$, наблюдения $y_{\tau}(\cdot)$ до момента $\tau$ и программы $u_{\tau}(\cdot)$ до момента $\tau$ введем множество

$$
\widehat{X}_{0}^{h}\left(\tau \mid y_{\tau}(\cdot), u_{\tau}(\cdot)\right)=\left\{x_{0} \in X_{0}:\left|s\left(x\left(t \mid x_{0}, u_{\tau}(\cdot)\right)\right)-y_{\tau}(t)\right| \leqslant h\left(t \in\left[t_{0}, \tau\right]\right)\right\}
$$

назовем его множеством началъных состояний, $h$-совместимых с наблюдением $y_{\tau}(\cdot)$ и программой $u_{\tau}(\cdot)$ до момента $\tau$. Отметим, что при увеличении $\tau$ данное множество, вообще говоря, сужается. Именно, очевидно следующее.

Лемма 4.1. Пусть $h>0, \delta \geqslant 0, \tau, \tau+\delta \in\left[t_{0}, \vartheta\right], y_{\tau}(\cdot) \in \mathscr{Y}_{\tau}, u_{\tau}(\cdot) \in \mathscr{U}_{\tau}$, $y_{\tau+\delta}(\cdot) \in \mathscr{Y}_{\tau+\delta}-$ продолжение $y_{\tau}(\cdot)$ на $\left[t_{0}, \tau+\delta\right] u u_{\tau+\delta}(\cdot) \in \mathscr{Y}_{\tau+\delta}-$ продолжение $u_{\tau}(\cdot)$ на $\left[t_{0}, \tau+\delta\right]$. Тогда

$$
\widehat{X}_{0}^{h}\left(\tau+\delta \mid y_{\tau+\delta}(\cdot), u_{\tau+\delta}(\cdot)\right) \subset \widehat{X}_{0}^{h}\left(\tau \mid y_{\tau}(\cdot), u_{\tau}(\cdot)\right) .
$$

Для всяких наблюдения $y_{\tau}(\cdot)$ до момента $\tau \in\left[t_{0}, \vartheta\right]$ и программы $u_{\tau}(\cdot)$ до $\tau$ построим множество

$$
\widehat{X}^{h}\left(\tau \mid y_{\tau}(\cdot), u_{\tau}(\cdot)\right)=\left\{x\left(\tau \mid x_{0}, u_{\tau}(\cdot)\right): x_{0} \in \widehat{X}_{0}^{h}\left(\tau \mid y_{\tau}(\cdot), u_{\tau}(\cdot)\right)\right\}
$$

назовем его информационным множеством, $h$-совместимым с наблюдением $y_{\tau}(\cdot)$ и программой $u_{\tau}(\cdot)$ в момент $\tau$. Ясно, что управляющая сторона в каждый момент $\tau$ может построить информационное множество (4.2) по истории $u_{\tau}(\cdot)$ своего управления, истории $y_{\tau}(\cdot)$ наблюдения сигнала (1.3) и значению $h$ параметра точности наблюдения. С точки зрения управляющей стороны данное множество есть совокупность всех точек фазового пространства системы (1.1), каждая из которых в момент $\tau$ подозрительна на то, чтобы быть истинной. Заметим, что при $\tau=t_{0}$ для $y_{t_{0}}(\cdot) \in \mathscr{Y}_{t_{0}}, u_{t_{0}}(\cdot) \in \mathscr{U}_{t_{0}}, h \geqslant 0$ имеем:

$$
\begin{aligned}
\widehat{X}^{h}\left(t_{0} \mid y_{t_{0}}(\cdot), u_{t_{0}}(\cdot)\right) & =\widehat{X}_{0}^{h}\left(t_{0} \mid y_{t_{0}}(\cdot), u_{t_{0}}(\cdot)\right) \\
& =\left\{x_{0} \in X_{0}:\left|s\left(x_{0}\right)-y_{t_{0}}\left(t_{0}\right)\right| \leqslant h\right\} .
\end{aligned}
$$


При управлении системой с помощью позиционной стратегии в условиях погрешностей наблюдения точности $h$ истинное состояние системы остается в информационном множестве, $h$-совместимом с текущей истрией наблюдения и текущей историей управления. Именно, верно следующее.

Лемма 4.2. Пусть $(u(\cdot), x(\cdot), y(\cdot))$ - управляемый процесс с начальным состоянием $x_{0}$ под действием позиционной стратегии $S$ при помехе наблюдения $\xi(\cdot)$ точности $h$. Тогда для всех $\tau \in\left[t_{0}, \vartheta\right]$ выполняется $x(\tau) \in \widehat{X}^{h}\left(\tau \mid y_{\tau}(\cdot)\right.$, $\left.u_{\tau}(\cdot)\right)$, где $y_{\tau}(\cdot)$ - сужение на $\left[t_{0}, \tau\right]$ функции $y(\cdot)$ и $u_{\tau}(\cdot)$ - сужение на $\left[t_{0}, \tau\right)$ функции и(·).

ДокАЗАТЕЛЬство. Пусть $\tau \in\left[t_{0}, \vartheta\right]$. По определению для управляемого процесса $(u(\cdot), x(\cdot), y(\cdot))$ выполняются соотношения $u(\cdot) \in \mathscr{U}, x(\cdot)=$ $x\left(\cdot \mid x_{0}, u(\cdot)\right), y(t)=s(x(t))+\xi(t)\left(t \in\left[t_{0}, \vartheta\right]\right)$. Поэтому $\mid s\left(x\left(t \mid x_{0}, u_{\tau}(\cdot)\right)\right)-$ $y_{\tau}(t) \mid \leqslant h\left(t \in\left[t_{0}, \tau\right]\right)$. Значит (см. (4.1)), $x_{0} \in \widehat{X}_{0}^{h}\left(\tau \mid y_{\tau}(\cdot), u_{\tau}(\cdot)\right)$. Следовательно, согласно $(4.2), x(\tau)=x\left(\tau \mid x_{0}, u_{\tau}(\cdot)\right) \in \widehat{X}_{0}^{h}\left(\tau \mid y_{\tau}(\cdot), u_{\tau}(\cdot)\right)$. Лемма 4.2 доказана.

Нас будет интересовать лишь содержательно значимая ситуация, когда наблюдение $y_{\tau}(\cdot)$ порождено каким-либо "реальным" движением и, соответственно, множество (4.2) непусто. Введем соответствующее определение. Скажем, что наблюдение $y_{\tau}(\cdot) \in \mathscr{Y}_{\tau} h$-допустимо в момент $\tau$ при программе $u_{\tau}(\cdot) \in$ $\mathscr{U}_{\tau}$, если существует $x_{0} \in X_{0}$ такое, что $\left|y(t)-s\left(x\left(t \mid x_{0}, u_{\tau}(\cdot)\right)\right)\right| \leqslant h$ при всех $t \in\left[t_{0}, \tau\right]$. Ясно, что информационное множество (4.2) непусто тогда и только тогда, когда наблюдение $y_{\tau}(\cdot) \quad h$-допустимо в момент $\tau$ при программе $u_{\tau}(\cdot)$.

Перейдем к описанию метода экстремального сдвига как правила назначения управляющей стороной значения $u_{*}$ управления в момент корректировки $\tau=\tau_{i}$ с расчетом удерживать это значение до следующего момента корректировки $\tau+\delta=\tau_{i+1}$. Это правило мы свяжем с $\eta(\cdot)$-мостом $(W(\tau))_{\tau \in\left[t_{0}, \vartheta\right]}$, порожденным каким-либо пакетом программ какой-либо точности $\bar{h}$ (в дальнейшем данный пакет программ будет выбираться $\varepsilon$-наводящим с достаточно малым $\varepsilon$ ). Грубый смысл выбора управления $u_{*}$ - максимально сдвинуть информационное множество $\widehat{E}(\tau)=\widehat{X}\left(\tau \mid y_{\tau}(\cdot), u_{\tau}(\cdot)\right)$ (восстановленное по историям $y_{\tau}(\cdot)$ наблюдения и $u_{\tau}(\cdot)$ управления) к ближайшему ("почти" ближайшему) к нему множеству $E(\tau)$ из класса $W(\tau)$. Такой экстремальный сдвиг реализуется при помощи аппроксимации всех движений, исходящих из всех допустимых начальных состояний $x_{0} \in X_{0}$, конечным числом движений, исходящих лишь из точек некоторой конечной (достаточно тонкой) сети множества $X_{0}$. Подобная аппроксимация позволяет ввести удобное аппроксимационное расстояние от информационного множества $\widehat{E}(\tau)$ - точнее, от его конечноточечной аппроксимации - до множеств $E(\tau) \in W(\tau)$. Удобством такого расстояния (хотя оно, не обладая свойством симметричности, не является расстоянием в строгом смысле) состоит в том, что оно имеет вид обычного евклидова расстояния между конечномерными векторами (достаточно большой размерности); это позволяет естественным образом определить как сопутствующее множество $E(\tau) \in W(\tau)$, ("почти") ближайшее к информационному множеству $\widehat{E}(\tau)$, так и "направление" от $\widehat{E}(\tau)$ - точнее, от его конечноточечной аппроксимации - к $E(\tau)$, вдоль которого, в обычном геометрическом смысле, и осуществляется желаемый максимальный сдвиг. 
Приступим к реализации обозначенной схемы. Прежде всего определим аппроксимационные расстояния от информационного множества (4.2) до множеств из фазового $\eta$-образа $W(\tau)$ в момент $\tau$ какого-либо пакета программ (см. (3.9)). Начнем с аппроксимации множества всех движений. Заметим, что из липшицевости правой части $f(\cdot)$ уравнения (1.1) (см. условия (А2) и (А3)) вытекает следующее: по всякому $\nu>0$ можно указать $\mu(\nu)>0$ такое, что для любых $x_{1}, x_{2} \in X_{0}$, удовлетворяющих неравенству $\left|x_{1}-x_{2}\right| \leqslant \mu(\nu)$, и для любой программы $u(\cdot)$ выполняется $\left|x\left(t \mid x_{1}, u(\cdot)\right)-x\left(t \mid x_{2}, u(\cdot)\right)\right| \leqslant \nu\left(t \in\left[t_{0}, \vartheta\right]\right)$. Для каждого $\nu>0$ мы зафиксируем конечную $\mu(\nu)$-сеть $X_{0}^{\nu}$ компакта $X_{0}$. Мы видим, что движения с произвольными допустимыми начальными состояниями аппроксимируются движениями с начальными состояниями из $X_{0}^{\nu}$ (при сохранении порождающих эти движения программ) с точностью $\nu$. Точнее, для всяких $\nu>0, x_{0} \in X_{0}$ найдется $x_{0}^{\nu} \in X_{0}^{\nu}$ такое, что при любой программе $u(\cdot)$ выполняется

$$
\left|x\left(t \mid x_{0}, u(\cdot)\right)-x\left(t \mid x_{0}^{\nu}, u(\cdot)\right)\right| \leqslant \nu \quad\left(t \in\left[t_{0}, \vartheta\right]\right) ;
$$

далее для всякой точки $x_{0} \in X_{0}$ мы зафиксируем какую-либо точку $x_{0}^{\nu} \in X_{0}^{\nu}$ с указанным свойством.

Сделаем следующее очевидное замечание, которым воспользуемся в дальнейшем. Пусть $\sigma(\cdot)$ - модуль непрерывности сигнальной функции $s(\cdot)$ на множестве $X$ (см. условие (А2)):

$$
\sigma(\delta)=\sup \left\{\left|s\left(x_{1}\right)-s\left(x_{2}\right)\right|: x_{1}, x_{2} \in X,\left|x_{1}-x_{2}\right| \leqslant \delta\right\} \quad(\delta \geqslant 0) .
$$

В силу непрерывности $s(\cdot)$ на $\mathbb{R}^{n}$ и ограниченности множества $X$ (см. условие $(\mathrm{A} 2))$ имеем: $\sigma(\delta) \rightarrow 0$ при $\delta \rightarrow 0$. Ввиду (4.4) для любых $\nu>0, x_{0} \in X_{0}$ и программы $u(\cdot)$ справедливо

$$
\left|s\left(x\left(t \mid x_{0}, u(\cdot)\right)\right)-s\left(x\left(t \mid x_{0}^{\nu}, u(\cdot)\right)\right)\right| \leqslant \sigma(\nu) \quad\left(t \in\left[t_{0}, \vartheta\right]\right) .
$$

При заданном $\nu>0$ в качестве конечноэлементной аппроксимации информационного множества $(4.2), h$-совместимого с наблюдением $y_{\tau}(\cdot) \in \mathscr{Y}_{\tau}$ и программой $u_{\tau}(\cdot) \in \mathscr{U}_{\tau}$ в момент $\tau$, будем рассматривать множество

$$
\widehat{X}^{h, \nu}\left(\tau \mid y_{\tau}(\cdot), u_{\tau}(\cdot)\right)=\left\{x\left(t \mid x_{0}^{\nu}, u_{\tau}(\cdot)\right): x_{0} \in \widehat{X}_{0}^{h}\left(\tau \mid y_{\tau}(\cdot), u_{\tau}(\cdot)\right)\right\} ;
$$

будем называть его $\nu$-аппроксимацией множества (4.2).

ЗАмЕчАНИЕ 4.1. Как следует из (4.4),

$$
\widehat{X}^{h}\left(\tau \mid y_{\tau}(\cdot), u_{\tau}(\cdot)\right) \subset\left[\widehat{X}^{h, \nu}\left(\tau \mid y_{\tau}(\cdot), u_{\tau}(\cdot)\right)\right]^{\nu} .
$$

Введем вспомогательное определение. Если $\widehat{D}$ и $D$ - непустые множества в $\mathbb{R}^{n}$, причем $\widehat{D}$ конечно, то расстоянием от $\widehat{D}$ до $D$ назовем число

$$
\rho(\widehat{D}, D)=\left(\sum_{\widehat{x} \in \widehat{D}} \operatorname{dist}(\widehat{x}, D)^{2}\right)^{1 / 2} ;
$$

здесь и далее

$$
\operatorname{dist}(\widehat{x}, D)=\inf _{x \in D}|\widehat{x}-x|
$$


Ключевыми объектами нашего дальнейшего анализа в данном разделе будут расстояния от $\nu$-аппроксимации информационного множества, $h$-совместимого с наблюдением $y_{\tau}(\cdot) \in \mathscr{Y}_{\tau}$ и программой $u_{\tau}(\cdot) \in \mathscr{U}_{\tau}$ в момент $\tau$, до множеств из фазового $\eta(\tau)$-образа $(3.10)$ в момент $\tau$ (при подходящей функции $\eta(\cdot)$ )

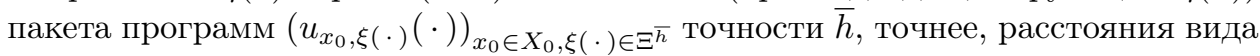
$\rho\left(\widehat{E}^{\nu}(\tau), E(\tau)\right)$, где $\widehat{E}^{\nu}(\tau)=\widehat{X}^{h, \nu}\left(\tau \mid y_{\tau}(\cdot), u_{\tau}(\cdot)\right)$ и $E(\tau)=X^{\eta(\tau)}\left(\tau \mid \bar{x}_{0}, \bar{\xi}(\cdot)\right)$ при некоторых $\bar{x}_{0} \in X_{0}, \bar{\xi}(\cdot) \in \Xi^{\eta(\tau)}$. Нас будет интересовать изменение расстояния $\rho\left(\widehat{E}^{\nu}(\tau), E(\tau)\right)$ при росте $\tau$. Наша цель в этом разделе - показать, что это расстояние имеет малую скорость возрастания в случае, когда к (неизвестному) "истинному" движению системы применяется управление, экстремально сдвигающее множество $\widehat{E}^{\nu}(\tau)$ на множество $E(\tau)$ (и при этом подходящим образом подбирается эволюция последнего множества).

Управление экстремального сдвига введем применительно к произвольному моменту времени $\tau \in\left[t_{0}, \vartheta\right]$ и к произвольным непустым множествам $\widehat{D}$ и $D$ из $\mathbb{R}^{n}$, где $\widehat{D}$ конечно. Именно, при заданном $\beta>0$ вектор $u_{*} \in P$ назовем управлением $\beta$-экстремального сдвига множества $\widehat{D}$ на множество $D$ в момент $\tau$, если $u_{*}$ - точка минимума на $P$ функции $\varphi(\cdot)$ вида

$$
\varphi(u)=\sum_{\widehat{x} \in \widehat{D}}\langle\widehat{x}-e(\widehat{x}), f(\tau, \widehat{x}, u)\rangle,
$$

где при каждом $\widehat{x} \in \widehat{D}$ элемент $e(\widehat{x}) \in D$ таков, что

$$
|\widehat{x}-e(\widehat{x})|^{2} \leqslant \operatorname{dist}(\widehat{x}, E(\tau))^{2}+\beta ;
$$

здесь и далее $\langle\cdot, \cdot\rangle$ - стандартное скалярное произведение в $\mathbb{R}^{n}$.

Результат данного раздела - следующая ниже лемма о локальной верхней оценке приращения квадрата расстояния $\rho^{2}\left(\widehat{E}^{\nu}(\tau), E(\tau)\right)$ при применении на малом отрезке времени $[\tau, \tau+\delta]$ управления $\beta$-экстремального сдвига множества $\widehat{E}^{\nu}(\tau)=\widehat{X}^{h, \nu}\left(\tau \mid y_{\tau}(\cdot), u_{\tau}(\cdot)\right)$ на множество $E(\tau)=X^{\eta(\tau)}\left(\tau \mid \bar{x}_{0}, \bar{\xi}(\cdot)\right)$ в момент $\tau$ (утверждение 3 ) леммы). В лемме, и всюду далее, используются следующие константы, которые мы зафиксируем: константа $K_{f}$ Липшица, общая для движений $x(\cdot)$, исходящих из всевозможных начальных состояний $x_{0} \in X_{0}$ под действием всевозможных программ $u(\cdot)$ (в качестве $K_{f}$ можно взять константу, ограничивающую сверху норму $|f(t, x, u)|$ при всех $t \in\left[t_{0}, \vartheta\right], x \in X$ и $u \in P)$; константа $K_{X}$, ограничивающая сверху нормы $|x(t)|\left(t \in\left[t_{0}, \vartheta\right]\right)$ для всех указанных выше движений; константа $L$ Липшица функции $f(\cdot)$ по фазовой переменной на множестве $X$ (это константа введена в условии (А3)). Кроме того, всюду далее $\mu(\cdot)$ - общий модуль непрерывности функций $t \mapsto f(t, x, u)$ $(x \in X, u \in P)$ :

$$
\mu(\delta)=\sup \left\{\left|f\left(t_{1}, x, u\right)-f\left(t_{2}, x, u\right)\right|: t_{1}, t_{2} \in\left[t_{0}, \vartheta\right],\left|t_{1}-t_{2}\right| \leqslant \delta, x \in X, u \in P\right\}
$$

$(\delta \geqslant 0)$; отметим, что в силу непрерывности функции $f(\cdot)$ и ограниченности множеств $X$ и $P$ имеем: $\mu(\delta) \rightarrow 0$ при $\delta \rightarrow 0$. Наконец, всюду далее символ $|E|$ обозначает число элементов конечного множества $E$.

ЛЕМмА 4.3. Пусть

(i) $\tau \in\left[t_{0}, \vartheta\right], h>0, \bar{h}>0, \nu>0$, 
(ii) наблюдение $y_{\tau}(\cdot) \in \mathscr{Y}_{\tau} h$-допустимо в момент $\tau$ при программе $u_{\tau}(\cdot) \in$ $\mathscr{U}_{\tau}, \widehat{E}(\tau)$ - информационное множество, $h$-совместимое с наблюдением $y_{\tau}(\cdot)$ и программой $u_{\tau}(\cdot)$ в момент $\tau$, m.е.

$$
\widehat{E}(\tau)=\widehat{X}^{h}\left(\tau, y_{\tau}(\cdot), u_{\tau}(\cdot)\right),
$$

$\bar{x}_{*}$ - произвольный элемент из $\widehat{E}(\tau)$ и множество $\widehat{E}^{\nu}(\tau)$ есть $\nu$-аппроксимация информационного множества $\widehat{E}(\tau)$, т.е.

$$
\widehat{E}^{\nu}(\tau)=\widehat{X}^{h, \nu}\left(\tau \mid y_{\tau}(\cdot), u_{\tau}(\cdot)\right),
$$

(iii) $\left(u_{x_{0}, \xi(\cdot)}(\cdot)\right)_{x_{0} \in X_{0}, \xi(\cdot) \in \Xi^{\bar{h}}}-$ nакет программ точности $\bar{h}, \eta(\tau) \in(0, \bar{h}] u$

$$
E(\tau)=X^{\eta(\tau)}\left(\tau \mid \bar{x}_{0}, \bar{\xi}(\cdot), u_{\bar{x}_{0}, \bar{\xi}(\cdot)}(\cdot)\right)
$$

при некоторьх $\bar{x}_{0} \in X_{0}, \bar{\xi}(\cdot) \in \Xi^{\eta(\tau)}$,

(iv) $\beta>0$ и $u_{*}$ - управление $\beta$-экстремального сдвига множества $\widehat{E}^{\nu}(\tau)$ на множество $E(\tau)$ в момент $\tau$,

(v) $\delta>0, \tau+\delta \in\left[t_{0}, \vartheta\right] \quad u \bar{x}_{* \delta}(\cdot)$ - решение (Каратеодори) на $\left[t_{0}, \tau+\delta\right]$ дифберенииального уравнения $\dot{x}(t)=f\left(t, x(t), u_{*}\right)$ (cм. (1.1)) такое, что $\bar{x}_{* \delta}(\tau)=\bar{x}_{*}$,

(vi) $y_{\tau+\delta}(\cdot) \in \mathscr{Y}_{\tau+\delta}-$ произвольное продолжение наблюдения $y_{\tau}(\cdot)$ на $\left[t_{0}, \tau+\delta\right]$ maкое, что

$$
\left|y_{\tau+\delta}(t)-s\left(\bar{x}_{* \delta}(t)\right)\right| \leqslant h \quad(t \in(\tau, \tau+\delta]),
$$

$u_{\tau+\delta}(\cdot) \in \mathscr{U}_{\tau+\delta}-$ продолжение программы $u_{\tau}(\cdot)$ на $\left[t_{0}, \tau+\delta\right]$ такое, что

$$
u_{\tau+\delta}(t)=u_{*} \quad(t \in[\tau, \tau+\delta)),
$$

(vii)

$$
\bar{d}=\rho^{2}\left(\widehat{E}^{\nu}(\tau), E(\tau)\right)(1+2 L \delta)+\left|X_{0}^{\nu}\right|(1+2 L \delta) \beta+C\left|X_{0}^{\nu}\right|(\delta+\mu(\delta)) \delta,
$$

¿de

$$
C=4 K_{f}\left(K_{X} L+1\right)+8 K_{X}
$$

(viii)

$$
\bar{h} \geqslant \eta(\tau+\delta) \geqslant \max \left\{\eta(\tau), 2\left(\sigma\left(\bar{d}^{1 / 2}\right)+h+\sigma(\nu)\right)\right\} .
$$

Тогда

1) наблюдение $y_{\tau+\delta}(\cdot) \quad h$-допустимо в момент $\tau+\delta$ при программе $u_{\tau+\delta}(\cdot)$

2) $\bar{x}_{* \delta}(\tau+\delta) \in \widehat{E}(\tau+\delta)$, где

$$
\widehat{E}(\tau+\delta)=\widehat{X}^{h}\left(\tau \mid y_{\tau+\delta}(\cdot), u_{\tau+\delta}(\cdot)\right),
$$

3) существуют $z_{0} \in X^{0} u \zeta(\cdot) \in \Xi^{\eta(\tau+\delta)}$ такие, что для множеств

$$
\begin{aligned}
\widehat{E}^{\nu}(\tau+\delta) & =\widehat{X}^{h, \nu}\left(\tau \mid y_{\tau+\delta}(\cdot), u_{\tau+\delta}(\cdot)\right), \\
E(\tau+\delta) & =X^{\eta(\tau+\delta)}\left(\tau \mid z_{0}, \zeta(\cdot), u_{z_{0}, \zeta(\cdot)}(\cdot)\right)
\end{aligned}
$$

справедлива оченка

$$
\rho^{2}\left(\widehat{E}^{\nu}(\tau+\delta), E(\tau+\delta)\right) \leqslant \bar{d}
$$


Доказательство леммы приведено в разделе 6.

Заметим, что утверждаемая основная оценка (4.19), при подстановке в ее правую часть явного выражения (4.13) для $\bar{d}$, имеет, в общем, вид локальной оценки, стандартной для теории позиционного управления с полной информацией о фазовых состояниях (см., например, параграф 14 монографии [6]). Особенности локальной оценки (4.19), связанные с неполнотой информации о фазовых состояниях, состоят в следующем. Во-первых, в оценке участвует точка $\bar{x}_{*}$, выделенная в информационом множестве $\widehat{E}(\tau)(4.9)$; эта точка - прототип неизвестного истинного состояния управляемой системы в момент $\tau$; управление $u_{*} \beta$-экстремального сдвига, применяемое для перемещения неизвестного истинного состояния, от этого состояния не зависит: оно зависит от доступной информации об истинном состоянии, т.е. от всего информационного множества $\widehat{E}(\tau)$ - точнее, от его аппроксимации $\widehat{E}^{\nu}(\tau)(4.10),-$ а также от сопутствующего множества $E(\tau)(4.11)$, связанного с выбранным пакетом программ, на которое и осуществляется $\beta$-экстремальный сдвиг множества $\widehat{E}^{\nu}(\tau)$. Во-вторых, оценке подвергается расстояние не между фазовыми точками, а между множествами новым информационным множеством, содержащим сдвинутое истинное состояние, - точнее, его аппроксимацией $\widehat{E}^{\nu}(\tau+\delta)(4.17)$ - и новым сопутствующим множеством $E(\tau+\delta)$ (4.18). В-третьих, локальная оценка (4.19) предполагает, что параметр точности $\eta(\tau+\delta)$ нового сопутствующего множества $E(\tau+\delta)$ (4.18), вообще говоря, увеличен по сравнению с параметром точности $\eta(\tau)$ исходного сопутствующего множества $E(\tau)(4.11)$ - см. (4.15); более того, степень этого увеличения (помимо зависимости от исходно заданных параметров $h$ и $\nu$ ) определяется, вообще говоря, величиной $\bar{d}$, записанной в правой части результирующей оценки (4.19) (от $\bar{d}$ зависит центральная часть (4.15), дающая нижнюю границу для $\eta(\tau+\delta))$; таким образом, локальная оценка увязывается с определенной, “достаточно сильной”, степенью расширения сопутствующего множества.

Заметим также, что в приводимом в разделе 6 доказательстве леммы 4.3 о локальной оценке свойство стабильности моста $(W(\tau))_{\tau \in\left[t_{0}, \vartheta\right]}$, порожденного

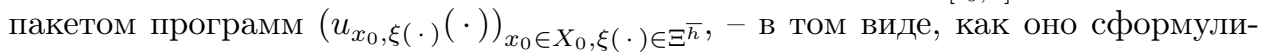
ровано в лемме 3.4, - формально не используется (как, собственно, и сам мост формально не присутствует в формулировке леммы, где участвуют лишь элементы $E(\tau) \in W(\tau)$ и $E(\tau+\delta) \in W(\tau+\delta))$. В ходе доказательства леммы (см. второй этап) нам оказывается удобнее непосредственно установить вариант свойства стабильности при специальном подборе параметров, приспособленном для получения локальной оценки (4.19). Таким образом, лемма 3.4 о стабильности, с формальной точки зрения, "не работает" на окончательный результат. Мы привели эту лемму и подробно остановились на ее "игровой" интерпретации (см. конец предыдущего раздела), руководствуясь соображениями методологического характера: во-первых, желая подчеркнуть аналогию нашей конструкции с соответствующей конструкцией из теории позиционных дифференциальных игр, во-вторых, стремясь продемонстрировать очищенную от деталей методологическую "заготовку", используемую, в усложненном виде, в весьма техническом доказательстве леммы 4.3 о локальной оценке. 


\section{5. Решение задачи позиционного наведения}

Опираясь на метод экстремального сдвига, описанный в предыдущем разделе, определим позиционную стратегию $S=\left(\tau_{i}, U_{i}\right)_{i=0}^{l+1}$ экстремального сдвига. Эту позиционную стратегию свяжем с $\eta(\cdot)$-мостом, порожденным неко-

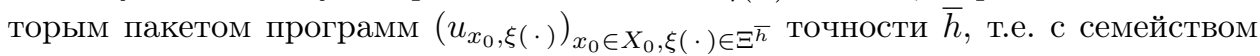

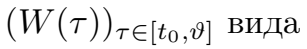

$$
W(\tau)=\left\{X^{\eta(\tau)}\left(\tau \mid \bar{x}_{0}, \bar{\xi}(\cdot), u_{\bar{x}_{0}, \bar{\xi}(\cdot)}(\cdot)\right):\left(\bar{x}_{0}, \bar{\xi}(\cdot)\right) \in X_{0} \times \Xi^{\eta(\tau)}\right\},
$$

где $\eta(\cdot)$ - какая-либо неотрицательная функция на $\left[t_{0}, \vartheta\right]$ со значениями, не превосходящими $\bar{h}$. Позиционную стратегию $S=\left(\tau_{i}, U_{i}\right)_{i=0}^{l+1}$ свяжем также с тремя положительными параметрами $-\nu, \beta$ и $\gamma$; первые два имеют тот же смысл, что и в предыдущем разделе ( $\nu$ - параметр аппроксимации информационных множеств, $\beta$ - параметр управления экстремального сдвига), а $\gamma$ характеризует способ выбора сопутствующих множеств $E\left(\tau_{i}\right)$ из класса $W\left(\tau_{i}\right)$ в моменты $\tau_{i}$ корректировки.

В содержательных терминах работа обратной связи $U_{i}$, определяемой стратегией $S$ в момент $\tau_{i}$ корректировки, может быть описана следующим образом. В момент $\tau_{i}$ управляющая сторона, зная параметр $h$ точности наблюдения, сложившуюся к моменту $\tau_{i}$ историю наблюдения $y_{\tau_{i}}(\cdot)$ и сложившуюся к этому моменту историю управления $u_{\tau_{i}}(\cdot)$, строит $\nu$-аппроксимацию

$$
\widehat{E}^{\nu}\left(\tau_{i}\right)=\widehat{X}^{h, \nu}\left(\tau_{i} \mid y_{\tau_{i}}(\cdot), u_{\tau_{i}}(\cdot)\right)
$$

информационного множества, $h$-совместимого с $y_{\tau_{i}}(\cdot)$ и $u_{\tau_{i}}(\cdot)$ в момент $\tau_{i}$. Затем в классе $W\left(\tau_{i}\right)$ управляющая сторона выбирает множество $E\left(\tau_{i}\right)$, "ближайшее с точностью $\gamma^{\prime \prime}$ к $\widehat{E}^{\nu}\left(\tau_{i}\right)$ в смысле квадрата расстояния $\rho(\cdot)(4.7)$ :

$$
\rho^{2}\left(\widehat{E}^{\nu}\left(\tau_{i}\right), E\left(\tau_{i}\right)\right) \leqslant \inf \left\{\rho^{2}\left(\widehat{E}^{\nu}\left(\tau_{i}\right), D\right): D \in W\left(\tau_{i}\right)\right\}+\gamma .
$$

Наконец, за значение $U_{i}\left(y_{\tau_{i}}(\cdot), u_{\tau_{i}}(\cdot)\right)$ обратной связи управляющая сторона принимает управление $u_{* i} \beta$-экстремального сдвига множества $\widehat{E}^{\nu}\left(\tau_{i}\right)$ на множество $E\left(\tau_{i}\right)$ в момент $\tau_{i}$.

Формальное определение таково. Пусть $\left(u_{x_{0}, \xi(\cdot)}\right)_{x_{0} \in X_{0}, \xi(\cdot) \in \Xi^{\bar{h}}}-$ пакет программ точности $\bar{h} \geqslant 0, \eta(\cdot)$ - неотрицательная функция на $\left[t_{0}, \vartheta\right]$ со значениями, не превосходящими $\bar{h}$, и $(W(\tau))_{\tau \in\left[t_{0}, \vartheta\right]}-\eta(\cdot)$-мост, порожденный пакетом программ $\left(u_{x_{0}, \xi(\cdot)}\right)_{x_{0} \in X_{0}, \xi(\cdot) \in \Xi^{\bar{h}}}$. Пусть также $\nu, \beta, \gamma>0$. Позиционную стратегию $S=\left(\tau_{i}, U_{i}\right)_{i=0}^{l+1}$ назовем стратегией $(\nu, \beta, \gamma)$-экстремального сдвига на $\eta(\cdot)$-мост $(W(\tau))_{\tau \in\left[t_{0}, \vartheta\right]}$, если для каждых $i=0, \ldots, l, u_{\tau_{i}}(\cdot) \in \mathscr{U}_{\tau_{i}}$ и $y_{\tau_{i}}(\cdot) \in \mathscr{Y}_{\tau_{i}}$ значение $U_{i}\left(y_{\tau_{i}}(\cdot), u_{\tau_{i}}(\cdot)\right)$ есть управление $\beta$-экстремального сдвига в момент $\tau_{i}$ множества $\widehat{E}^{\nu}\left(\tau_{i}\right)$ на какое-либо множество $E\left(\tau_{i}\right) \in W\left(\tau_{i}\right)$, удовлетворяющее условию (5.2).

С помощью леммы 4.3 о локальной оценке мы установим, что при подходящем подборе параметров стратегия $(\nu, \beta, \gamma)$-экстремального сдвига на $\eta(\cdot)$-мост $(W(\tau))_{\tau \in\left[t_{0}, \vartheta\right]}$ удерживает аппроксимации $\widehat{E}^{\nu}\left(\tau_{i}\right)$ информационных множеств, содержащих истинные состояния системы, вблизи классов $W\left(\tau_{i}\right)$.

Рассмотрим сначала начальный момент времени $t_{0}$. 
Лемма 5.1. Пусть $(W(\tau))_{\tau \in\left[t_{0}, \vartheta\right]}-\eta(\cdot)$-мост, порожденный пакетом программ $\left(u_{x_{0}, \xi(\cdot)}\right)_{x_{0} \in X_{0}, \xi(\cdot) \in \Xi^{h}}$ точности $\bar{h}, h \geqslant 0, \nu>0$ u $\eta\left(t_{0}\right) \geqslant 2 h+\sigma(\nu)$. Тогда для любого наблюдения $y_{t_{0}}(\cdot) \in \mathscr{Y}_{t_{0}}, h$-допустимого в момент $t_{0}$, сущеcтвует $E\left(t_{0}\right) \in W\left(t_{0}\right)$ такое, что множество $\widehat{E}^{\nu}\left(t_{0}\right)=\widehat{X}^{h, \nu}\left(t_{0} \mid y_{t_{0}}(\cdot), u_{t_{0}}(\cdot)\right)$ удовлетворяет условию $\rho\left(\widehat{E}^{\nu}\left(t_{0}\right), E\left(t_{0}\right)\right)=0$.

ДоказАтельство. Так как наблюдение $y_{t_{0}}(\cdot) \in \mathscr{Y}_{t_{0}} h$-допустимо в момент $t_{0}$, то $\left|y_{t_{0}}\left(t_{0}\right)-s\left(\bar{x}_{0}\right)\right| \leqslant h$ при некотором $\bar{x}_{0} \in X_{0}$. Положим $E\left(t_{0}\right)=\left\{x_{0} \in\right.$ $\left.X_{0}:\left|s\left(x_{0}\right)-s\left(\bar{x}_{0}\right)\right| \leqslant \eta\left(t_{0}\right)\right\}$. Согласно $(3.2), E\left(t_{0}\right)=X^{\eta\left(t_{0}\right)}\left(t_{0} \mid \bar{x}_{0}, 0, \bar{u}(\cdot)\right)$, т.е. $E\left(t_{0}\right) \in W\left(t_{0}\right)$. По $(4.3)$

$$
\widehat{X}^{h}\left(t_{0} \mid y_{t_{0}}(\cdot), u_{t_{0}}(\cdot)\right)=\left\{x_{0} \in X_{0}:\left|s\left(x_{0}\right)-y_{t_{0}}\left(t_{0}\right)\right| \leqslant h\right\} .
$$

Тогда в соответствии с определением $\nu$-апроксимации $\widehat{E}^{\nu}\left(t_{0}\right)($ см. $(4.6))$

$$
\begin{aligned}
\widehat{E}^{\nu}\left(t_{0}\right) & =\left\{x_{0}^{\nu}: x_{0} \in \widehat{X}^{h}\left(t_{0} \mid y_{t_{0}}(\cdot), u_{t_{0}}(\cdot)\right)\right\} \\
& =\left\{x_{0}^{\nu}: x_{0} \in X_{0},\left|s\left(x_{0}\right)-y_{t_{0}}\left(t_{0}\right)\right| \leqslant h\right\} .
\end{aligned}
$$

Возьмем произвольный элемент $\widehat{x} \in \widehat{E}^{\nu}\left(t_{0}\right)$. Тогда $\widehat{x}=x_{0}^{\nu}$ для некоторого $x_{0} \in X_{0}$ такого, что $\left|s\left(x_{0}\right)-y_{t_{0}}\left(t_{0}\right)\right| \leqslant h$. Поскольку $\left|y_{t_{0}}\left(t_{0}\right)-s\left(\bar{x}_{0}\right)\right| \leqslant h$, то $\left|s\left(x_{0}\right)-s\left(\bar{x}_{0}\right)\right| \leqslant 2 h$. Из определения аппроксимирующей точки $x_{0}^{\nu}$ (см. (4.5)) $\left|s(\widehat{x})-s\left(x_{0}\right)\right|=\left|s\left(x_{0}^{\nu}\right)-s\left(x_{0}\right)\right| \leqslant \sigma(\nu)$. Отсюда и из предыдущего неравенства $\left|s(\widehat{x})-s\left(\bar{x}_{0}\right)\right| \leqslant 2 h+\sigma(\nu) \leqslant \eta\left(t_{0}\right)$. Таким образом, $\widehat{x} \in E\left(t_{0}\right)$. В силу произвольности $\widehat{x} \in \widehat{E}\left(t_{0}\right)$ имеем вложение $\widehat{E}^{\nu}\left(t_{0}\right) \subset E\left(t_{0}\right)$, влекущее равенство $\rho\left(\widehat{E}^{\nu}\left(t_{0}\right), E\left(t_{0}\right)\right)=0$. Лемма 5.1 доказана.

При применении стратегии $(\nu, \beta, \gamma)$-экстремального сдвига на $\eta(\cdot)$-мост $(W(\tau))_{\tau \in\left[t_{0}, \vartheta\right]}$ нас будет интересовать изменение квадрата расстояния от $\nu$-аппроксимаций $\widehat{E}^{\nu}\left(\tau_{i}\right)$ текущих информационных множеств до классов $W\left(\tau_{i}\right)$, т.е. величины

$$
\omega_{i}=\inf \left\{\rho^{2}\left(\widehat{E}^{\nu}\left(\tau_{i}\right), D\right): D \in W\left(\tau_{i}\right)\right\} .
$$

Локальная оценка (4.19) (с учетом вида ее правой части (4.13)) подсказывает нам, что при подходящем подборе параметров подобная же оценка должна сохраниться и для $\omega_{i}$, иными словами, можно ожидать выполнения неравенств

$$
\omega_{i+1} \leqslant \omega_{i}\left[1+2 L\left(\tau_{i+1}-\tau_{i}\right)\right]+a_{i}
$$

с некоторыми $a_{i} \geqslant 0$, подобными сумме последних двух слагаемых из правой части (4.13). При этом, как указывает лемма 5.1, для $i=0$, соответствующего начальному моменту $\tau_{0}=t_{0}$, можно обеспечить $\omega_{0}=0$. Для дальнейшего нам удобно, в предположении, что эти соотношения выполнены, заранее оценить величины $\omega_{i}$ сверху. Индукцией легко проверить следующее.

Лемма 5.2. Пусть $t_{0}=\tau_{0}<\cdots<\tau_{p+1} \leqslant \vartheta, \omega_{0}=0$ и для всех $i=0, \ldots, k$, где $k \leqslant p$, выполняется (5.3). Тогда

$$
\omega_{i+1} \leqslant e^{2 L\left(\tau_{i}-t_{0}\right)}\left(a_{0}+\cdots+a_{i}\right) \quad(i=1, \ldots, k) .
$$


По лемме 4.3 для справедливости локальной оценки (4.19) требуется, чтобы значение "нового параметра совместимости" $\eta(\tau+\delta)$ оценивалось снизу некоторым выражением, зависящим от "старого параметра совместимости" $\eta(\tau)$ и от правой части локальной оценки (4.19) (см. (4.15)). Формальный перенос нижней оценки (4.15) в применении к величинам $\omega_{i}$ ведет к нижним оценкам вида

$$
\eta\left(\tau_{i+1}\right) \geqslant \max \left\{\eta\left(\tau_{i}\right), 2\left(\sigma\left(\omega_{i+1}^{1 / 2}\right)+h+\sigma(\nu)\right)\right\}
$$

или, с учетом (5.4), к более сильным оценкам

$$
\eta\left(\tau_{i+1}\right) \geqslant \max \left\{\eta\left(\tau_{i}\right), 2\left(\sigma\left(\bar{\omega}_{i+1}^{1 / 2}\right)+h+\sigma(\nu)\right)\right\},
$$

где

$$
\bar{\omega}_{i+1}=e^{2 L\left(\tau_{i+1}-t_{0}\right)}\left(a_{0}+\cdots+a_{i}\right) .
$$

Покажем, что при принятии этих ограничений и начального ограничения $\eta\left(t_{0}\right) \geqslant 2 h+\sigma(\nu)$ из леммы 5.1 верхние оценки (5.3) (и вытекающие из них оценки (5.4) или, что то же, $\left.\omega_{i} \leqslant \bar{\omega}_{i}\right)$ действительно справедливы, причем при подходящем подборе параметров сумма $a_{0}+\cdots+a_{l}$ сколь угодно мала.

ЛЕмма 5.3. Пусть

(i) $S=\left(\tau_{i}, U_{i}\right)_{i=0}^{l+1}$ - стратегия $(\nu, \beta, \gamma)$-экстремального сдвига на $\eta(\cdot)$-мост $(W(\tau))_{\tau \in\left[t_{0}, \vartheta\right]}$, порожденный пакетом программ $\left(u_{x_{0}, \xi(\cdot)}\right)_{x_{0} \in X_{0}, \xi(\cdot) \in \Xi^{\bar{h}}}$ точности $\bar{h} \geqslant \sup _{t \in\left[t_{0}, \vartheta\right]} \eta(t)$,

(ii) $h>0$ и при всех $i=0, \ldots, l$

$$
\bar{\omega}_{i+1}=e^{2 L\left(\tau_{i+1}-t_{0}\right)}\left(a_{0}+\cdots+a_{i}\right)
$$

где

$$
\begin{gathered}
a_{i}=\left|X_{0}^{\nu}\right|\left[1+2 L\left(\tau_{i+1}-\tau_{i}\right)\right] \beta \\
+C\left|X_{0}^{\nu}\right|\left[\left(\tau_{i+1}-\tau_{i}\right)+\mu\left(\tau_{i+1}-\tau_{i}\right)\right]\left(\tau_{i+1}-\tau_{i}\right) \\
+\left[1+2 L\left(\tau_{i+1}-\tau_{i}\right)\right] \gamma, \\
\quad C=4 K_{f}\left(K_{X} L+1\right)+8 K_{X},
\end{gathered}
$$

(iii) функиия $\eta(\cdot)$, определяющая $\eta(\cdot)$-мост $(W(\tau))_{\tau \in\left[t_{0}, \vartheta\right]}$, удовлетворяет условиям

$$
\begin{gathered}
\eta\left(t_{0}\right) \geqslant 2 h+\sigma(\nu), \\
\eta\left(\tau_{i+1}\right) \geqslant \max \left\{\eta\left(\tau_{i}\right), 2\left(\sigma\left(\bar{\omega}_{i+1}^{1 / 2}\right)+h+\sigma(\nu)\right)\right\} \quad(i=0, \ldots, l),
\end{gathered}
$$

(iv) $(u(\cdot), x(\cdot), y(\cdot))$ - управляемый процесс с некоторым начальным состоянием под действием позиционной стратегии $S$ при некоторой помехе наблюдения точности $h$,

(v) для каждого $i=0, \ldots, l+1 u_{\tau_{i}}(\cdot)$ - сужение $u(\cdot)$ на $\left[t_{0}, \tau_{i}\right), y_{\tau_{i}}(\cdot)$

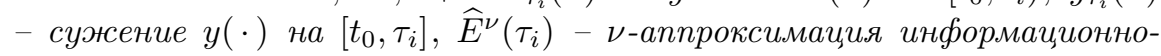
го множества, $h$-совместимого с $y_{\tau_{i}}(\cdot)$ и $u_{\tau_{i}}(\cdot)$ в момент $\tau_{i}$, m.e. $\widehat{E}^{\nu}\left(\tau_{i}\right)=\widehat{X}^{h, \nu}\left(\tau_{i} \mid y_{\tau_{i}}(\cdot), u_{\tau_{i}}(\cdot)\right), u$

$$
\omega_{i}=\inf \left\{\rho^{2}\left(\widehat{E}^{\nu}\left(\tau_{i}\right), D\right): D \in W\left(\tau_{i}\right)\right\} .
$$


Тогда для всех $i=0, \ldots, l$ верны оценки

$$
\begin{gathered}
\omega_{i+1} \leqslant \omega_{i}\left[1+2 L\left(\tau_{i+1}-\tau_{i}\right)\right]+a_{i}, \\
\omega_{i+1} \leqslant \bar{\omega}_{i+1} .
\end{gathered}
$$

ДоКАЗАТЕЛЬСТВО. Положим $\omega_{-1}=a_{-1}=0$ и покажем (5.10) и (5.11) для $i=-1,0, \ldots, l$. Применим индукцию. Вследствие (5.7) по лемме $5.1 \omega_{0}=0$. Поэтому для $i=-1$ оценки (5.10) и (5.11) справедливы. Предположим, что (5.10) и (5.11) верны при всех $i=-1, \ldots, k-1$, где $k-1<l$, и покажем, что

$$
\begin{gathered}
\omega_{k+1} \leqslant \omega_{k}\left[1+2 L\left(\tau_{k+1}-\tau_{k}\right)\right]+a_{k}, \\
\omega_{k+1} \leqslant \bar{\omega}_{k+1} .
\end{gathered}
$$

В силу предположения индукции и леммы 5.2 при выполнении (5.12) верно и (5.13). Осталось показать справедливость неравенства (5.12). По определению позиционной стратегии $S$ имеем $u(t)=u_{* k} \quad\left(t \in\left[\tau_{k}, \tau_{k+1}\right)\right)$, где $u_{* k}=$ $U_{k}\left(y_{\tau_{k}}(\cdot), u_{\tau_{k}}(\cdot)\right)$ - управление $\beta$-экстремального сдвига в момент $\tau_{k}$ множества $\widehat{E}^{\nu}\left(\tau_{k}\right)$ на некоторое множество $E\left(\tau_{k}\right) \in W\left(\tau_{k}\right)$, удовлетворяющее условию

$$
\rho^{2}\left(\widehat{E}^{\nu}\left(\tau_{k}\right), E\left(\tau_{k}\right)\right) \leqslant \omega_{k}+\gamma
$$

(см. (5.9)). Проверим выполнение условий леммы 4.3 о локальной оценке, полагая $\tau=\tau_{k}, \bar{x}_{*}=x\left(\tau_{k}\right), u_{*}=u_{* k}, \delta=\tau_{k+1}-\tau_{k}$. Замечая, что по лемме $4.2 x\left(\tau_{k}\right) \in \widehat{E}\left(\tau_{k}\right)=\widehat{X}^{h}\left(\tau_{k} \mid y_{\tau_{k}}(\cdot), u_{\tau_{k}}(\cdot)\right)$, получаем, что условия (i)-(iv) леммы 4.3 выполняются. Условие $(\mathrm{v})$, очевидно, выполняется при $\bar{x}_{* \delta}(t)=x(t)$ $\left(t \in\left[\tau_{k}, \tau_{k+1}\right]\right)$. Выполнение условия (vi) следует из определения управляемого процесса $(u(\cdot), x(\cdot), y(\cdot))$. Условие (vii) мы выполним, задав

$$
\bar{d}=\rho^{2}\left(\widehat{E}^{\nu}\left(\tau_{k}\right), E\left(\tau_{k}\right)\right)\left[1+2 L\left(\tau_{k+1}-\tau_{k}\right)\right]+c
$$

при

$$
\begin{aligned}
c & =\left|X_{0}^{\nu}\right|\left[1+2 L\left(\tau_{k+1}-\tau_{k}\right)\right] \beta+C\left|X_{0}^{\nu}\right|\left[\left(\tau_{i+1}-\tau_{i}\right)+\mu\left(\tau_{i+1}-\tau_{i}\right)\right]\left(\tau_{i+1}-\tau_{i}\right) \\
& =a_{k}-\left[1+2 L\left(\tau_{k+1}-\tau_{k}\right)\right] \gamma
\end{aligned}
$$

(последнее равенство следует из (5.6)). Обратимся к условию (viii) леммы 4.3. С учетом (5.14) и предположения индукции $\omega_{k} \leqslant \bar{\omega}_{k}$ имеем:

$$
\begin{aligned}
\bar{d} & \leqslant\left(\omega_{k}+\gamma\right)\left[1+2 L\left(\tau_{k+1}-\tau_{k}\right)\right]+a_{k}-\left[1+2 L\left(\tau_{k+1}-\tau_{k}\right)\right] \gamma \\
& \leqslant \bar{\omega}_{k}\left[1+2 L\left(\tau_{k+1}-\tau_{k}\right)\right]+a_{k} .
\end{aligned}
$$

Подставляя выражение для $\bar{\omega}_{k}($ см. (5.5)), продолжим:

$$
\begin{aligned}
\bar{d} & \leqslant e^{2 L\left(\tau_{k}-t_{0}\right)}\left(a_{0}+\cdots+a_{k-1}\right)\left[1+2 L\left(\tau_{k+1}-\tau_{k}\right)\right]+a_{k} \\
& \leqslant e^{2 L\left(\tau_{k+1}-t_{0}\right)}\left(a_{0}+\cdots+a_{k-1}\right)+e^{2 L\left(\tau_{k+1}-t_{0}\right)} a_{k} \\
& =\bar{\omega}_{k+1} .
\end{aligned}
$$

Поэтому, из-за монотонного возрастания модуля непрерывности $\sigma(\cdot)$, оценка (5.8) при $i=k$, т.е. оценка

$$
\eta\left(\tau_{k+1}\right) \geqslant \max \left\{\eta\left(\tau_{k}\right), 2\left(\sigma\left(\bar{\omega}_{k+1}^{1 / 2}\right)+h+\sigma(\nu)\right)\right\}
$$


влечет неравенство

$$
\eta\left(\tau_{k+1}\right) \geqslant \max \left\{\eta\left(\tau_{k}\right), 2\left(\sigma\left(\bar{d}^{1 / 2}\right)+h+\sigma(\nu)\right)\right\} .
$$

В принятых обозначениях последнее неравенство есть оценка (4.15). Справедливость данной оценки означает выполнение последнего условия (viii) леммы 4.3. Итак, все условия леммы 4.3 выполнены. По этой лемме существуют $z_{0} \in X^{0}$ и $\zeta(\cdot) \in \Xi^{\eta\left(\tau_{k+1}\right)}$ такие, что для

$$
E\left(\tau_{k+1}\right)=X^{\eta\left(\tau_{k+1}\right)}\left(\tau_{k+1} \mid z_{0}, \zeta(\cdot), u_{z_{0}, \zeta(\cdot)}(\cdot)\right)
$$

верно неравенство

$$
\rho^{2}\left(\widehat{E}^{\nu}\left(\tau_{k+1}\right), E\left(\tau_{k+1}\right)\right) \leqslant \bar{d} .
$$

Ясно, что $E\left(\tau_{k+1}\right) \in W\left(\tau_{k+1}\right)$ (см. (5.1)). Поэтому из определения $\omega_{k+1}$ (см. (5.9)) с использованием (5.15) имеем:

$$
\begin{aligned}
\omega_{k+1} & \leqslant \bar{d} \leqslant\left(\omega_{k}+\gamma\right)\left[1+2 L\left(\tau_{k+1}-\tau_{k}\right)\right]+a_{k}-\left[2 L\left(\tau_{k+1}-\tau_{k}\right)\right] \gamma \\
& =\omega_{k}\left[1+2 L\left(\tau_{k+1}-\tau_{k}\right)\right]+a_{k} .
\end{aligned}
$$

Оценка (5.12) установлена. Лемма 5.3 доказана.

Чтобы извлечь из леммы 5.3 результат об обращении теоремы 2.1, следует заметить следующее.

Лемма 5.4. Для каждого положителъного $h$ можно указать положительные $\nu=\nu(h), \quad \beta=\beta(h), \quad \gamma=\gamma(h), \quad$ точки $\tau_{i}=\tau_{i}(h) \in\left[t_{0}, \vartheta\right]$ $(i=0, \ldots, l+1=l(h)+1), t_{0}=\tau_{0}<\cdots<\tau_{l+1}=\vartheta$, неотрицательную фбункиию $\eta(\cdot)=\eta(\cdot \mid h)$ на $\left[t_{0}, \vartheta\right]$ и положительное $\bar{h}=\bar{h}(h)$ такие, что

(i) указанные параметры удовлетворяют всем условиям леммы 5.3, именно, $\eta(\cdot)$ принимает значения в $[0, \bar{h}]$ и подчиняется оценкам (5.7), (5.8) при $\bar{\omega}_{i+1}=\bar{\omega}_{i+1}(h)(i=0, \ldots, l)$, определенных по (5.5), (5.6),

(ii) выполняются равенства

$$
\begin{gathered}
\lim _{h \rightarrow 0} \max _{i=0, \ldots, l(h)} \bar{\omega}_{i+1}(h)=0, \quad \lim _{h \rightarrow 0} \bar{h}(h)=0, \\
\lim _{h \rightarrow 0} \nu(h)=0, \quad \lim _{h \rightarrow 0} \beta(h)=0, \quad \lim _{h \rightarrow 0} \gamma(h)=0 .
\end{gathered}
$$

ДокАЗАТЕЛьСтво. Возьмем произвольное $h>0$. Приведем один из возможных способов задания желаемых параметров. Положим $\bar{h}=\bar{h}(h)=3 h$, тем самым сразу обеспечивая второе равенство (5.16). Зададим положительные $\nu=\nu(h)$ и $\bar{\omega}^{0}=\bar{\omega}^{0}(h)$ так, что

$$
2\left(\sigma\left(\left(\bar{\omega}^{0}\right)^{1 / 2}\right)+\sigma(\nu)\right) \leqslant h .
$$

Ясно, что

$$
\lim _{h \rightarrow 0} \bar{\omega}^{0}(h)=0
$$

и верно первое равенство в (5.17). Заметим, что вследствие (5.18) имеем:

$$
\bar{h}_{1}=2\left(\sigma\left(\left(\bar{\omega}^{0}\right)^{1 / 2}\right)+h+\sigma(\nu)\right) \leqslant 3 h=\bar{h} .
$$


По $\nu=\nu(h)$ зададим $\delta=\delta(h)$ так, что

$$
b_{1}=e^{2 L\left(\vartheta-t_{0}\right)} C\left|X_{0}^{\nu}\right|\left(\vartheta-t_{0}\right)(\delta+\mu(\delta)) \leqslant \bar{\omega}^{0} / 2 .
$$

Заметим, что в силу (5.19)

$$
\lim _{h \rightarrow 0} \delta(h)=0 .
$$

Возьмем $\tau_{i}=\tau_{i}(h)(i=0, \ldots, l+1=l(h)+1), t_{0}=\tau_{0}<\cdots<\tau_{l+1}=\vartheta$, такими, что $\max _{i=0, \ldots, l}\left(\tau_{i+1}-\tau_{i}\right) \leqslant \delta$. Выберем положительные $\beta=\beta(h)$ и $\gamma=\gamma(h)$ так, что

$$
b_{2}=e^{2 L\left(\vartheta-t_{0}\right)}\left[(l+1)\left|X_{0}^{\nu}\right|\left[1+2 L\left(\vartheta-t_{0}\right)\right] \beta+(l+1)\left[1+2 L\left(\vartheta-t_{0}\right)\right] \gamma\right] \leqslant \bar{\omega}^{0} / 2
$$

заметим, что в силу (5.19) верно второе и третье равенство в (5.17). Тогда, используя для $a_{i}(5.6)$ оценки

$$
\begin{aligned}
& a_{i} \leqslant \bar{a}_{i}=\left|X_{0}^{\nu}\right|\left[1+2 L\left(\vartheta-t_{0}\right)\right] \beta+C\left|X_{0}^{\nu}\right|\left(\tau_{i+1}-\tau_{i}\right)(\delta+\mu(\delta))+\left[1+2 L\left(\vartheta-t_{0}\right)\right] \gamma \\
& \text { и для } \bar{\omega}_{i+1}(5.5) \text { оценки }
\end{aligned}
$$

$$
\bar{\omega}_{i+1} \leqslant e^{2 L\left(\vartheta-t_{0}\right)}\left(\bar{a}_{0}+\cdots+\bar{a}_{i}\right)
$$

получаем:

$$
\bar{\omega}_{i+1} \leqslant b_{1}+b_{2} \leqslant \bar{\omega}^{0} \quad(i=0, \ldots, l) .
$$

Вследствие (5.19) обеспечивается (5.16). Остается указать функцию $\eta(\cdot)=$ $\eta(\cdot \mid h)$ на $\left[t_{0}, \vartheta\right]$, принимающую значения в $[0, \bar{h}]$ и удовлетворяющую неравенствам (5.7) и (5.8). Зададим $\eta(\cdot)$ как произвольную неубывающую функцию на $\left[t_{0}, \vartheta\right]$, принимающую значения в $\left[\bar{h}_{1}, \bar{h}\right]$ (этот отрезок непуст из-за $(5.20)$ ). Как следует из (5.22) и неубывания модуля непрерывности $\sigma(\cdot)$, записанное в правой части (5.8) выражение $2\left(\sigma\left(\bar{\omega}_{i+1}^{1 / 2}\right)+h+\sigma(\nu)\right)$ при любом $h \in\left[0, h^{0}\right]$ не превосходит числа $\bar{h}_{1}(5.20)$. Отсюда ясно, что и записанное в правой части $(5.7)$ выражение $2 h+\sigma(\nu)$ также не превосходит $\bar{h}_{1}$ при любом $h \in\left[0, h^{0}\right]$. Поэтому $\eta(\cdot)$ удовлетворяет неравенствам (5.7) и (5.8). Лемма 5.4 доказана.

При каждом положительном $h$ для указанных в лемме 5.4 параметров введем следующие наименовния: $\nu=\nu(h)$ - согласованный с $h$ napaмemp anпроксимации движений, $\beta=\beta(h)$ - согласованный с $h$ параметр управления, $\gamma=\gamma(h)$ - согласованный с $h$ параметр сопутствующего множества, $\tau_{i}=\tau_{i}(h)(i=0, \ldots, l+1=l(h)+1)$ - согласованные с $h$ моменты корректировки, $\bar{h}=\bar{h}(h)$ - согласованный с $h$ сопутствующий параметр точности, $\eta(\cdot)=\eta(\cdot \mid h)$ - согласованная с $h$ сопутствующая функция точности.

Опираясь на леммы 5.3 и 5.4, построим семейство стратегий экстремального сдвига, разрешающее задачу позиционного наведения при разрешимости задачи пакетного наведения. Тем самым мы получим желаемое обращение теоремы 2.1 .

Напомним, что при разрешимости задачи пакетного наведения существует монотонно наводящее семейство пакетов программ (лемма 2.1). 
TEOPEMA 5.1. Пусть

(i) разрешима задача пакетного наведения $u\left(\left(u_{\bar{x}_{0}, \bar{\xi}(\cdot)}\right)_{\left.\bar{x}_{0} \in X_{0}, \bar{\xi}(\cdot) \in \Xi^{h^{*}(\varepsilon)}\right)_{\varepsilon>0}}\right.$ - монотонно наводящее семейство пакетов программ,

(ii) для каждого $h>0 \nu(h)$ - согласованный $c h$ параметр аппроксимации движений, $\beta(h)$ - согласованный с $h$ параметр управления, $\gamma(h)$ согласованный с $h$ параметр сопутствующего множества, $\tau_{i}(h)(i=$ $0, \ldots, l(h)+1)$ - согласованные с $h$ моменты корректировки, $\bar{h}(h)$ - согласованный с $h$ сопутствующий параметр точности и $\eta(\cdot \mid h)$ - согласованная с $h$ сопутствующая функиия точности,

(iii) для каждого $h>0$ такого, что $\bar{h}(h) \geqslant \sup _{\varepsilon \in(0,1)} h^{*}(\varepsilon), S_{h}-$ произвольная позиционная стратегия,

(iv) для каждого $h>0$ такого, что $\bar{h}(h) \in\left(0, \sup _{\varepsilon \in(0,1)} h^{*}(\varepsilon)\right), \quad S_{h}=$ $\left(\tau_{i}^{h}, U_{i}^{h}\right)_{i=0}^{l^{h}+1} \quad$ - стратегия $(\nu(h), \beta(h), \gamma(h))$-экстремального сдвига на $\eta(\cdot \mid h)$-мост $\left(W^{h}(\tau)\right)_{\tau \in\left[t_{0}, \vartheta\right]}, \quad$ порожденный пакетом программ $\left(u_{\bar{x}_{0}, \bar{\xi}(\cdot)}^{\overline{\bar{c}(h)}}\right)_{\bar{x}_{0} \in X_{0}, \bar{\xi}(\cdot) \in \Xi^{\bar{h}(h)}}$ точности $\bar{h}(h)$, где

$$
\bar{\varepsilon}(h) \in \Pi(h)=\left\{\varepsilon \in(0,1): \bar{h}(h) \leqslant h^{*}(\varepsilon)\right\}, \quad \bar{\varepsilon}(h)<\inf \Pi(h)+h,
$$

u при этом $\tau_{i}^{h}=\tau_{i}(h)\left(i=0, \ldots, l^{h}+1=l(h)+1\right)$.

Тогда семейство $\left(S_{h}\right)_{h>0}$ позичионных стратегий является наводящим.

ДокАЗАтЕЛьСтво. Поскольку параметры $\nu=\nu(h), \beta=\beta(h), \gamma=\gamma(h)$, $\tau_{i}(h)(i=0, \ldots, l+1=l(h)+1), \bar{h}=\bar{h}(h)$ и $\eta(\cdot)=\eta(\cdot \mid h)$ согласованы с $h$, по лемме 5.4 заключаем, что они удовлетворяют всем условиям леммы 5.3 и, кроме того, для $\bar{\omega}_{i+1}=\bar{\omega}_{i+1}(h)(i=0, \ldots, l)$, определенных по $(5.5),(5.6)$, выполняется

$$
\lim _{h \rightarrow 0} \max _{i=0, \ldots, l(h)} \bar{\omega}_{i+1}(h)=0
$$

(см. (5.16)). Возьмем произвольное $\varepsilon_{0} \in(0,1)$. Отметим $h_{1}>0$ такое, что

$$
\max _{i=0, \ldots, l(h)} \bar{\omega}_{i+1}(h)<\left(\varepsilon_{0} / 4\right)^{2} \quad\left(h \in\left(0, h_{1}\right)\right) .
$$

По лемме $5.4 \lim _{h \rightarrow 0} \nu(h)=0$. Зафиксируем $h_{2}>0$ такое, что

$$
\nu(h)<\varepsilon_{0} / 4 \quad\left(h \in\left(0, h_{2}\right)\right) .
$$

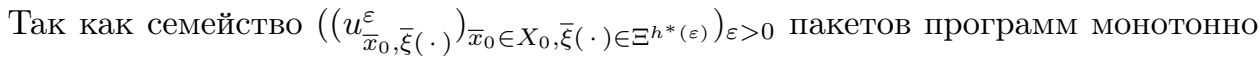
наводящее, функция $h^{*}(\cdot)$ возрастает, и, значит,

$$
h^{*}\left(\varepsilon_{0} / 4\right) \leqslant \sup _{\varepsilon \in(0,1)} h^{*}(\varepsilon) .
$$

Поскольку по лемме $5.4 \lim _{h \rightarrow 0} \bar{h}(h)=0$, то найдется $h_{3}>0$ такое, что

$$
\bar{h}(h)<h^{*}\left(\varepsilon_{0} / 4\right) \quad\left(h \in\left(0, h_{3}\right)\right) .
$$

Возьмем произвольное

$$
h \in\left(0, \min \left\{h_{1}, h_{2}, h_{3}, \varepsilon_{0} / 4\right\}\right) .
$$


Расмотрим управляемый процесс $(u(\cdot), x(\cdot), y(\cdot))$ с произвольным начальным состоянием под действием позиционной стратегии $S_{h}$ при произвольной помехе наблюдения точности $h$. Для завершения доказательства достаточно показать, что

$$
x(\vartheta) \in[M]^{\varepsilon_{0}} .
$$

Покажем это. Из (5.27) и (5.26) видим, что позиционная стратегия $S_{h}=$ $\left(\tau_{i}^{h}, U_{i}^{h}\right)_{i=0}^{l^{h}+1}$ имеет вид, указанный в условии (iv), т.е. является стратегией $(\nu(h), \beta(h), \gamma(h))$-экстремального сдвига на $\eta(\cdot \mid h)$-мост $\left(W^{h}(\tau)\right)_{\tau \in\left[t_{0}, \vartheta\right]}$, порожденный пакетом программ $\left(u_{\bar{x}_{0}, \bar{\xi}(\cdot)}^{\overline{\bar{c}(h)}}\right)_{\bar{x}_{0} \in X_{0}, \bar{\xi}(\cdot) \in \Xi^{\bar{h}(h)}}$, и при этом $\tau_{i}^{h}=\tau_{i}(h)(i=$ $\left.0, \ldots, l^{h}+1=l(h)+1\right)$. Из того, что указанные выше параметры удовлетворяют условиям леммы 5.3, заключаем, что условиям леммы 5.3 удовлетворяют и определяемые этими параметрами пакет программ

$$
\left(u_{\bar{x}_{0}, \bar{\xi}(\cdot)}\right)_{\bar{x}_{0} \in X_{0}, \bar{\xi}(\cdot) \in \Xi^{\bar{h}}}=\left(u_{\bar{x}_{0}, \bar{\xi}(\cdot)}^{\bar{\varepsilon}(h)}\right)_{\bar{x}_{0} \in X_{0}, \bar{\xi}(\cdot) \in \Xi^{\bar{h}(h)}},
$$

порожденный им $\eta(\cdot)$-мост $(W(\tau))_{\tau \in\left[t_{0}, \vartheta\right]}=\left(W^{h}(\tau)\right)_{\tau \in\left[t_{0}, \vartheta\right]}$ и стратегия $S=S_{h}$ $(\nu, \beta, \gamma)$-экстремального сдвига на $(W(\tau))_{\tau \in\left[t_{0}, \vartheta\right]}$. Применяя лемму 5.3 , получаем, что, каковы бы ни были $x_{0} \in X_{0}$ и $\xi(\cdot) \in \Xi^{h}$, для управляемого процесса $(u(\cdot), x(\cdot), y(\cdot))$ с начальным состоянием $x_{0}$ под действием позиционной стратегии $S_{h}$ при помехе наблюдения $\xi(\cdot)$ выполняется

$$
\inf \left\{\rho^{2}\left(\widehat{E}^{\nu}\left(\tau_{i}\right), D\right): D \in W\left(\tau_{i}\right)\right\} \leqslant \bar{\omega}_{i} \quad(i=1, \ldots, l+1)
$$

(см. (5.9) и (5.11)); здесь $\widehat{E}^{\nu}\left(\tau_{i}\right)-\nu$-аппроксимация информационного множества, $h$-совместимого с $y_{\tau_{i}}(\cdot)$ и $u_{\tau_{i}}(\cdot)$ в момент $\tau_{i}$, и $y_{\tau_{i}}(\cdot)$ и $u_{\tau_{i}}(\cdot)-$ соотвественно сужения на $\left[t_{0}, \tau_{i}\right]$ и на $\left[t_{0}, \tau_{i}\right)$ наблюдения $y(\cdot)$ и программы $u(\cdot)$. В частности, с учетом (5.24) имеем:

$$
\inf \left\{\rho^{2}\left(\widehat{E}^{\nu}(\vartheta), D\right): D \in W\left(\tau_{i}\right)\right\} \leqslant \bar{\omega}_{l}<\left(\varepsilon_{0} / 4\right)^{2} .
$$

По лемме $4.2 x(\vartheta) \in \widehat{X}^{h}(\vartheta \mid y(\cdot), u(\cdot))$. Отсюда по замечанию $4.1 \quad x(\vartheta) \in$ $\left[\widehat{E}^{\nu}(\vartheta)\right]^{\nu}$. Поэтому вследствие $(5.25) x(\vartheta) \in\left[\widehat{E}^{\nu}(\vartheta)\right]^{\varepsilon_{0} / 4}$. Обращаясь к (5.31), получаем, что найдется $E \in W(\vartheta)$ такое, что $x(\vartheta) \in[E]^{\varepsilon_{0} / 2}$. Для справедливости включения (5.29) осталось установить, что

$$
E \subset[M]^{\varepsilon_{0} / 2} .
$$

Установим это, рассмотрев пакет программ $\left(u_{\bar{x}_{0}, \bar{\xi}(\cdot)}^{\bar{s}(h)}\right)_{\bar{x}_{0} \in X_{0}, \bar{\xi}(\cdot) \in \Xi^{\bar{h}(h)}}$, порождающий $\eta(\cdot)$-мост $(W(\tau))_{\tau \in\left[t_{0}, \vartheta\right]}$. Так как $\bar{h}(h)<h^{*}\left(\varepsilon_{0} / 4\right)$ (см. (5.27)), то $\varepsilon_{0} / 4 \in \Pi(h)$ (см. (5.23)), и по определению $\bar{\varepsilon}(h)(5.23)$ имеем $\bar{\varepsilon}(h) \leqslant \varepsilon_{0} / 4+h$. Отсюда, поскольку $h<\varepsilon_{0} / 4$ (см. $\left.(5.28)\right), \bar{\varepsilon}(h) \leqslant \varepsilon_{0} / 2$. Поэтому $\bar{\varepsilon}(h)$-наводя-

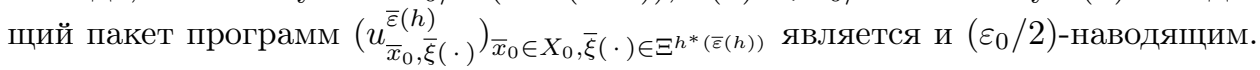
Поскольку по определению (5.23) $\bar{\varepsilon}(h) \in \Pi(\varepsilon)$, то $\bar{h}(h) \leqslant h^{*}(\bar{\varepsilon}(h))$. Значит, пакет программ $\left(u_{\bar{x}_{0}, \bar{\xi}(\cdot)}^{\bar{\varepsilon}(h)+h}\right)_{\bar{x}_{0} \in X_{0}, \bar{\xi}(\cdot) \in \Xi^{\bar{h}(h)}}$ точности $\bar{h}(h)$ есть сужение $\left(\varepsilon_{0} / 2\right)$-наводящего пакета программ $\left(u_{\bar{x}_{0}, \bar{\xi}(\cdot)}^{\bar{\varepsilon}(h)}\right)_{\bar{x}_{0} \in X_{0}, \bar{\xi}(\cdot) \in \Xi^{h^{*}(\bar{\varepsilon}(h))}}$ точности $h^{*}(\bar{\varepsilon}(h))$. Следовательно, по замечанию 2.1 он также является $\left(\varepsilon_{0} / 2\right)$-наводящим. Так как 
$E \in W(\vartheta),(W(\tau))_{\tau \in\left[t_{0}, \vartheta\right]}$ есть $\eta(\cdot)$-мост, порожденный данным $\left(\varepsilon_{0} / 2\right)$-наводящим пакетом программ точности $\bar{h}(h)$, и $\eta(\vartheta) \leqslant \bar{h}(h)$, то по лемме 3.3 верно вложение (5.32). Теорема 5.1 доказана.

Из теорем 2.1 и 5.1 извлекаем окончательное утверждение об эквивалентности задачи позиционного наведения и задачи пакетного наведения.

ТЕОРема 5.2. Задача позиционного наведения разрешима тогда и только тогда, когда разрешима задача пакетного наведния. Точнее, наводящее семейство позиционных стратегий существует тогда и только тогда, когда для каждого $\varepsilon>0$ существует в-наводящий пакет программ некоторой положительной точности.

\section{6. Доказательство леммы о локальной оценке}

В этом разделе мы приводим доказательство центральной леммы 4.3 о локальной оценке. Предполагаем, что выполнены все условия этой леммы. В целях ясности изложения сначала опишем схему доказательства неформально.

Первый этап. Для каждой точки $\widehat{x}_{*}$ из информационного множества $\widehat{E}(\tau)$ $(4.9)$ строим движение $\widehat{x}_{*}(\cdot)$, сдвигающее эту точку от момента $\tau$ до момента $\tau+\delta$ в силу управления $u_{*} \beta$-экстремального сдвига (см. условие (iv) леммы). Одно из таких движений, $\bar{x}_{*}(\cdot)$, производит сдвиг точки $\bar{x}_{*}$, выделенной в условии (ii); сдвинутая точка $\bar{x}_{*}(\tau+\delta)$ (что почти очевидно из свойства (4.12) наблюдения $\left.y_{\tau+\delta}(\cdot)\right)$ попадает в новое информационное множество $\widehat{E}(\tau+\delta)(4.16)$; тем самым доказываются утверждения 1 и 2 леммы. Параллельно с движениями $\widehat{x}_{*}(\cdot)$ строим аппроксимирующие движения $\widehat{x}_{*}^{[\nu]}(\cdot)$, сдвигающие подобным же образом аппроксимирующие точки $\widehat{x}_{*}^{[\nu]}$, принадлежащие $\nu$-аппроксимации $\widehat{E}^{\nu}(\tau)$ (4.10) информационного множества $\widehat{E}(\tau)$. Сдвинутые аппроксимирующие точки $\widehat{x}_{*}^{[\nu]}(\tau+\delta)$ заметают некоторое конечное множество, которое, вообще говоря, шире $\nu$-аппроксимации $\widehat{E}^{\nu}(\tau+\delta)(4.17)$ информационного множества $\widehat{E}(\tau+\delta)$ : часть точек $\widehat{x}_{*}^{[\nu]}(\tau+\delta)$ высекается за счет вновь поступивших наблюдений $y_{\tau+\delta}(t)(t \in[\tau, \tau+\delta])$. При этом одна из этих точек, а именно точка $\bar{x}_{*}^{[\nu]}(\tau+\delta)-$ сдвинутая аппроксимация $\bar{x}_{*}^{[\nu]}$ выделенной точки $\bar{x}_{*}-$ заведомо попадает в $\widehat{E}^{\nu}(\tau+\delta)$, это есть следствие того факта, что $\bar{x}_{*}(\tau+\delta) \in \widehat{E}^{\nu}(\tau+\delta)$.

Второй этап. Для аппроксимирующих точек $\widehat{x}=\widehat{x}_{*}^{[\nu]}(\cdot)$, покрывающих множество $\widehat{E}^{\nu}(\tau)$, берем “почти ближайшие" (“ближайшие с точностью $\beta$ ”) элементы $e(\widehat{x})$ множества $E(\tau)(4.11)$, определяющие, в соответствии со схемой $(4.8)$, управление $u_{*} \beta$-экстремального сдвига. Среди этих точек мы отмечаем точку $\bar{w}_{*}=e\left(\bar{x}_{*}^{[\nu]}\right)$, соответствующую выделенной аппроксимирующей точке $\bar{x}_{*}^{[\nu]} \in$ $\widehat{E}^{\nu}(\tau)$. Точка $\bar{w}_{*}$ служит для определения программы $v(\cdot)$, задающей трансформацию множества $E(\tau)$ в множество $E(\tau+\delta)(4.18)$. В качестве $v(\cdot)$ берем тот элемент пакета программ $\left(u_{x_{0}, \xi(\cdot)}(\cdot)\right)_{x_{0} \in X_{0}, \xi(\cdot) \in \Xi^{\bar{h}}}$ (см. условие (iii) леммы), который приводит систему в момент $\tau$ в точку $\bar{w}_{*}$. Параметры $z_{0}$ (начальная точка) и $\zeta(\cdot)$ (помеха наблюдения) этого элемента пакета программ используются при формальном определении множества $E(\tau+\delta)$. Точка $\bar{w}_{*}$ после сдвига 
в силу программы $v(\cdot)$ до момента $\tau+\delta$ естественным образом попадает в "подстроенное под нее" множество $E(\tau+\delta)$. Параллельно с помощью программы $v(\cdot)$ мы сдвигаем до момента $\tau+\delta$ все точки $e(\widehat{x})$.

Третий этап. На этом этапе анализируется пучок движений $z(\cdot \mid \widehat{x})$, построенных на первом этапе, и пучок движений $w(\cdot \mid \widehat{x})$, построенных на втором этапе: движения $z(\cdot \mid \widehat{x})$ перемещают до момента $\tau+\delta$ все точки $\widehat{x} \in \widehat{E}^{\nu}(\tau)$ с применением управления $u_{*} \quad \beta$-экстремального сдвига, а движения $z(\cdot \mid \widehat{x})$ перемещают до момента $\tau+\delta$ все точки $e(\widehat{x}) \in E(\tau)$ с применением программы $v(\cdot)$. Используя стандартную технику экстремального сдвига, показываем, что в соответствующем произведении евклидовых пространств квадрат расстояния $d(\tau+\delta)$ между этими пучками движений, подсчитанного в момент $\tau+\delta$, удовлетворяет оценке типа желаемой финальной оценки $(4.19): d(\tau+\delta) \leqslant \bar{d}$.

Четвертый этап. Здесь реализуется основной прием, отражающий специфику применения метода экстремального сдвига для случая неполной информации о фазовых состояниях. Как отмечалось выше (второй этап), набор сдвинутых точек $z(\tau+\delta \mid \widehat{x})$ избыточен в том смысле, что при переборе всех $\widehat{x} \in \widehat{E}^{\nu}(\tau)$ точки $z(\tau+\delta \mid \widehat{x})$ заметают множество, которое, вообще говоря, шиpe, чем аппроксимационное информационное множество $\widehat{E}^{\nu}(\tau+\delta)$ (тогда как нам необходимо оценить сверху расстояние именно от этого аппроксимационного информационного множества до множества $E(\tau+\delta))$. Из этого избыточного набора точек отбираем точки $z(\tau+\delta \mid \widehat{x})$, покрывающие в точности множество $\widehat{E}^{\nu}(\tau+\delta)$. Параллельно автоматически отбираются сооответствующие точки $w(\tau+\delta \mid \widehat{x})$. Квадрат расстояния $d_{\delta}(\tau+\delta)$ между наборами отобранных точек, разумеется, не превосходит квадрата расстояния между их полными наборами и, таким образом, подчиняется оценке желаемого типа, подобной той, которая была установлена в конце третьего этапа: $d_{\delta}(\tau+\delta) \leqslant \bar{d}$. Для завершения доказательства остается лишь показать, что все отобранные точки $w(\tau+\delta \mid \widehat{x})$ лежат в $E(\tau+\delta)$. Здесь мы существенно пользуемся двумя фактами. Первый факт состоит в том, что на втором этапе множество $E(\tau+\delta)$ построено таким образом, что точка $w(\tau+\delta \mid \widehat{x})=w\left(\tau+\delta \mid \bar{x}_{*}^{[\nu]}\right)$, заведомо попадающая в $E(\tau+\delta)$, получена сдвигом (по программе $v(\cdot))$ элемента $\bar{w}_{*}=e\left(\bar{x}_{*}^{[\nu]}\right) \in E(\tau)$, "почти ближайшего" к точке $\bar{x}_{*}^{[\nu]}$. Второй факт состоит в том, что точка $z\left(\tau+\delta \mid \bar{x}_{*}^{[\nu]}\right)$, полученная сдвигом (с помощью управления $u_{*}$ ) точки $\bar{x}_{*}^{[\nu]}$, заведомо лежит в $\widehat{E}(\tau+\delta)$. Таким образом, мы получаем "базовую" пару отобранных точек, одна из которых (по принципу отбора) лежит в $\widehat{E}^{\nu}(\tau+\delta)$, а другая (по построению) - в $E(\tau+\delta)$; при этом квадрат расстояния между этими точками, понятно, не превосходит квадрата расстояния $d_{\delta}(\tau+\delta)$ между наборами всех отобранных точек $z(\tau+\delta \mid \widehat{x})$ и $w(\tau+\delta \mid \widehat{x})$. Это дает возможность оценить сверху - через $d_{\delta}(\tau+\delta) \leqslant \bar{d}$ (и ряд сопутствующих параметров) - отклонение значений $s(w(t))$, реализующихся вдоль произвольного отобранного движения $w(t)=w(\tau+\delta \mid \widehat{x})$, от значений $s(\bar{w}(t))$, реализующихся вдоль “базового" движения $\bar{w}(t)=w\left(\tau+\delta \mid \bar{x}_{*}^{[\nu]}\right)$ для $t \in[\tau, \tau+\delta]$. Приняв полученную верхнюю оценку за параметр точности $\eta(\tau+\delta)$ для множества $E(\tau+\delta)$ (4.18) (это заранее заложено в условия леммы - см. (4.15)), заключаем, что все отобранные точки $w(\tau+\delta \mid \widehat{x})$ лежат в $E(\tau+\delta)$.

Переходим к строгому доказательству. 
Первый этап. Возьмем произвольную точку $\widehat{x}_{*} \in \widehat{E}(\tau)$. Согласно определению множества $\widehat{E}(\tau)$ (см. (4.9) и $(4.2))$,

$$
\widehat{x}_{*}=x\left(\tau \mid \widehat{x}_{* 0}, u_{\tau}(\cdot)\right)
$$

при некотором $\widehat{x}_{* 0} \in \widehat{E}_{0}(\tau)$, где

$$
\widehat{E}_{0}(\tau)=\widehat{X}_{0}^{h}\left(\tau \mid y_{\tau}(\cdot), u_{\tau}(\cdot)\right) .
$$

По начальной точке $\widehat{x}_{* 0}$ построим аппроксимирующую ее начальную точку $\widehat{x}_{* 0}^{\nu} \in X_{0}^{\nu}$ и введем движения

$$
\widehat{x}_{*}(\cdot)=x\left(\cdot \mid \widehat{x}_{* 0}, u_{\tau+\delta}(\cdot)\right), \quad \widehat{x}_{*}^{[\nu]}(\cdot)=x\left(\cdot \mid \widehat{x}_{* 0}^{\nu}, u_{\tau+\delta}(\cdot)\right)
$$

(напомним, что продолжение $u_{\tau+\delta}(\cdot) \in \mathscr{U}_{\tau+\delta}$ программы $u_{\tau}$ определено в условии (vi)). Заметим, что по определению аппроксимирующей начальной точки $\widehat{x}_{* 0}^{\nu}$ выполняется

$$
\left|\widehat{x}_{*}^{[\nu]}(t)-\widehat{x}_{*}(t)\right| \leqslant \nu \quad\left(t \in\left[t_{0}, \tau+\delta\right]\right) .
$$

Ниже также будет использовано вытекающее из (6.4) неравенство (см. (4.5))

$$
\left|s\left(\widehat{x}_{*}^{[\nu]}(t)\right)-s\left(\widehat{x}_{*}(t)\right)\right| \leqslant \sigma(\nu) \quad\left(t \in\left[t_{0}, \tau+\delta\right]\right) .
$$

С учетом первого из обозначений (6.3) перепишем (6.1) в виде

$$
\widehat{x}_{*}=\widehat{x}_{*}(\tau) .
$$

Тот факт, что $\widehat{x}_{* 0}=\widehat{x}_{*}\left(t_{0}\right) \in \widehat{E}_{0}(\tau)$, означает, что

$$
\left|s\left(\widehat{x}_{*}(t)\right)-y_{\tau}(t)\right| \leqslant h \quad\left(t \in\left[t_{0}, \tau\right]\right) .
$$

Введем в рассмотрение точку

$$
\widehat{x}_{*}^{[\nu]}=x\left(\tau \mid x_{* 0}^{\nu}, u_{\tau}(\cdot)\right)=\widehat{x}_{*}^{[\nu]}(\tau) .
$$

По определению множества $\widehat{E}^{\nu}(\tau)$ (см. (4.10)) имеем $\widehat{x}^{[\nu]} \in \widehat{E}^{\nu}(\tau)$.

Итак, для произвольной точки $\widehat{x}_{*} \in \widehat{E}(\tau)$ мы определили следующие зависящие от нее объекты: начальную точку $\widehat{x}_{* 0} \in \widehat{E}_{0}(\tau)$; ее аппроксимацию $\widehat{x}_{* 0}^{[\nu]} \in X_{0}^{\nu}$; движение $\widehat{x}_{*}(\cdot)$ до момента $\tau+\delta$, исходящее из начального состояния $\widehat{x}_{* 0} \in X_{0}$ под действием программы $u_{\tau+\delta}(\cdot)$ и проходящее в момент $\tau$ через точку $\widehat{x}_{*}$; аппроксимирующее движение $\widehat{x}_{*}^{[\nu]}(\cdot)$ до момента $\tau+\delta$, исходящее из аппроксимирующего начального состояния $\widehat{x}_{* 0}^{\nu} \in X_{0}$ под действием программы $u_{\tau+\delta}(\cdot)$ и проходящее в момент $\tau$ через точку $\widehat{x}_{*}^{[\nu]} \in \widehat{E}^{\nu}(\tau)$. Основные свойства построенных аппроксимаций, связанных с точкой $\widehat{x}_{*}$, выражены неравенствами (6.4) и (6.5). Заметим, что если перебрать все точки $\widehat{x}_{*} \in \widehat{E}(\tau)$, то соответствующие точки $\widehat{x}_{*}^{[\nu]} \in \widehat{E}^{\nu}(\tau)$ покроют все множество $\widehat{E}^{\nu}(\tau)$, а точки $\widehat{x}_{* 0} \in \widehat{E}_{0}(\tau)$ покроют все множество $\widehat{E}_{0}(\tau)$ :

$$
\begin{aligned}
\left\{\widehat{x}_{*}^{[\nu]}: \widehat{x}_{*} \in \widehat{E}(\tau)\right\} & =\widehat{E}^{\nu}(\tau), \\
\left\{\widehat{x}_{* 0}: \widehat{x}_{*} \in \widehat{E}(\tau)\right\} & =\widehat{E}_{0}(\tau) ;
\end{aligned}
$$


это легко следует из определений точек $\widehat{x}_{*}^{[\nu]} \in \widehat{E}^{\nu}(\tau)$ (см. (6.8)) и множества $\widehat{E}^{\nu}(\tau)($ см. $(4.10))$, а также определений точек $\widehat{x}_{* 0}($ см. $(6.1))$ и множества $\widehat{E}_{0}(\tau)$ (см. (6.2)).

Рассмотрим в качестве $\widehat{x}_{*}$ точку $\bar{x}_{*} \in \widehat{E}(\tau)$, выделенную в условии (ii). Для

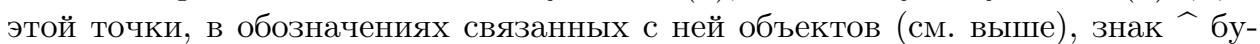
дем заменять на - , именно, будем полагать: $\widehat{x}_{* 0}=\bar{x}_{* 0}, \widehat{x}_{* 0}^{[\nu]}=\bar{x}_{* 0}^{[\nu]}, \widehat{x}_{*}(\cdot)=$ $\bar{x}_{*}(\cdot), \widehat{x}_{*}^{[\nu]}(\cdot)=\bar{x}_{*}^{[\nu]}(\cdot), \widehat{x}_{*}^{[\nu]}=\bar{x}_{*}^{[\nu]}$. Заметим, что при этом сужение $\bar{x}_{*}(\cdot)$ на $[\tau, \tau+\delta]$ есть решение на этом отрезке дифференциального уравнения $\dot{x}(t)=$ $f\left(t, x(t), u_{*}\right)$ с начальным условием $\bar{x}_{*}(\tau)=\bar{x}_{*}$ и, таким образом, совпадает с функцией $\bar{x}_{\delta *}(\cdot)$, определенной в условии $(\mathrm{v})$ :

$$
\bar{x}_{*}(t)=\bar{x}_{\delta *}(t) \quad(t \in[\tau, \tau+\delta]) .
$$

В частности, условие (4.12) можно переписать как

$$
\left|s\left(\bar{x}_{*}(t)\right)-y_{\tau+\delta}(t)\right| \leqslant h \quad(t \in(\tau, \tau+\delta]) .
$$

Условие (6.7) в применении к точке $\bar{x}_{*}$ принимает вид

$$
\left|s\left(\bar{x}_{*}(t)\right)-y_{\tau}(t)\right| \leqslant h \quad\left(t \in\left[t_{0}, \tau\right]\right),
$$

что вместе с (4.12) дает:

$$
\left|s\left(\bar{x}_{*}(t)\right)-y_{\tau+\delta}(t)\right| \leqslant h \quad\left(t \in\left[t_{0}, \tau+\delta\right]\right) .
$$

Так как $\bar{x}_{*}(\cdot)=x\left(\cdot \mid \bar{x}_{* 0}, u_{\tau+\delta}(\cdot)\right)($ см. (6.3)), то (6.13) означает, что, во-первых, наблюдение $y_{\tau+\delta}(\cdot) \quad h$-допустимо в момент $\tau+\delta$ при программе $u_{\tau+\delta}(\cdot)$ и, вовторых,

$$
\bar{x}_{*}(\tau+\delta)=\bar{x}_{\delta *}(\tau+\delta) \in \widehat{E}(\tau+\delta),
$$

где $\widehat{E}(\tau+\delta)$ (см. (4.16)) - информационное множество, $h$-совместимое с наблюдением $y_{\tau+\delta}(\cdot)$ и программой $u_{\tau+\delta}(\cdot)$ в момент $\tau+\delta$. Тем самым доказаны утверждения 1) и 2) настоящей леммы.

Сосредоточимся на доказательстве основного утверждения 3). Получим нужное для дальнейшего представление для $\nu$-аппроксимации $\widehat{E}^{\nu}(\tau+\delta)(4.17)$ информационного множества $\widehat{E}(\tau+\delta)(4.16)$. Положим

$$
\widehat{E}_{0}(\tau+\delta)=\widehat{X}_{0}^{h}\left(\tau \mid y_{\tau+\delta}(\cdot), u_{\tau+\delta}(\cdot)\right) .
$$

По лемме 4.1 и по $(6.10)$

$$
\widehat{E}_{0}(\tau+\delta) \subset \widehat{E}_{0}(\tau)=\left\{\widehat{x}_{* 0}: \widehat{x}_{*} \in \widehat{E}(\tau)\right\} .
$$

Заметим, что из определения множества $\widehat{E}_{0}(\tau+\delta)$ (см. также $\left.(4.1)\right)$ и определения движений $\widehat{x}_{*}(\cdot)$ (см. (6.3)) имеем:

$$
\left|s\left(\widehat{x}_{*}(t)\right)-y_{\tau+\delta}(t)\right| \leqslant h \quad\left(t \in\left[t_{0}, \tau+\delta\right], \quad \widehat{x}_{* 0} \in \widehat{E}_{0}(\tau+\delta)\right) .
$$

Из (6.15), с использованием определения информационного множества $\widehat{E}(\tau+\delta)$ (см. (4.16) и (4.2)) и движений $\widehat{x}_{*}(\cdot)($ см. (6.3)), получаем:

$$
\widehat{E}(\tau+\delta)=\left\{\widehat{x}_{*}(\tau+\delta): \widehat{x}_{* 0} \in \widehat{E}_{0}(\tau+\delta)\right\} .
$$


Тогда для $\nu$-аппроксимации $\widehat{E}^{\nu}(\tau+\delta)$ (4.17) информационного множества $\widehat{E}(\tau+\delta)$, используя ее определение (см. (4.6)), имеем:

$$
\widehat{E}^{\nu}(\tau+\delta)=\left\{\widehat{x}_{*}^{[\nu]}(\tau+\delta): \widehat{x}_{* 0} \in \widehat{E}_{0}(\tau+\delta)\right\}
$$

или

$$
\widehat{E}^{\nu}(\tau+\delta)=\left\{\widehat{x}_{*}^{[\nu]}(\tau+\delta): \widehat{x}_{* 0}^{\nu} \in \widehat{E}_{0}^{\nu}(\tau+\delta)\right\}
$$

где

$$
\widehat{E}_{0}^{\nu}(\tau+\delta)=\left\{\widehat{x}_{* 0}^{\nu}: \widehat{x}_{* 0} \in \widehat{E}_{0}(\tau+\delta)\right\}
$$

В силу (6.15)

$$
\widehat{E}_{0}^{\nu}(\tau+\delta) \subset \widehat{E}_{0}^{\nu}(\tau)=\left\{\widehat{x}_{* 0}^{\nu}: \widehat{x}_{* 0} \in \widehat{E}_{0}(\tau)\right\} .
$$

Наконец, из (6.5) и (6.16) получаем:

$$
\left|s\left(\widehat{x}_{*}^{[\nu]}(t)\right)-y_{\tau+\delta}(t)\right| \leqslant h+\sigma(\nu) \quad\left(t \in\left[t_{0}, \tau+\delta\right], \quad \widehat{x}_{* 0} \in \widehat{E}_{0}(\tau+\delta)\right) .
$$

Соотношениями (6.17), (6.18) и (6.19) воспользуемся в дальнейшем.

Второй этап. Обратимся к элементам, участвующим в задании управления $u_{*} \beta$-экстремального сдвига множества $\widehat{E}^{\nu}(\tau)$ на множество $E(\tau)$ в момент $\tau$ (см. условие (iv)). По определению, $u_{*}$ минимизирует на $P$ значение

$$
\varphi(u)=\sum_{\widehat{x} \in \widehat{E}^{\nu}(\tau)}\langle\widehat{x}-e(\widehat{x}), f(\tau, \widehat{x}, u)\rangle,
$$

где при каждом $\widehat{x} \in \widehat{E}^{\nu}(\tau)$ элемент $e(\widehat{x}) \in E(\tau)$ таков, что

$$
|\widehat{x}-e(\widehat{x})|^{2} \leqslant \operatorname{dist}(\widehat{x}, E(\tau))^{2}+\beta .
$$

Далее для каждого $\widehat{x} \in \widehat{E}^{\nu}(\tau)$ зафиксируем элемент $e(\widehat{x}) \in E(\tau)$.

Теперь мы подберем некоторую специальную программу $v(\cdot)$ и с ее помощью сдвинем точки $e(\widehat{x})$, где $\widehat{x} \in \widehat{E}^{\nu}(\tau)$, от момента $\tau$ до момента $\tau+\delta$. Рассмотрим элемент

$$
\bar{w}_{*}=e\left(\bar{x}_{*}^{[\nu]}\right) ;
$$

напомним, что $\bar{x}_{*}^{[\nu]} \in \widehat{E}^{\nu}(\tau)$ - введенная ранее аппроксимация элемента $\bar{x}_{*} \in$ $\widehat{E}(\tau)$, выделенного в условии (ii). По определению множества $E(\tau)$ (см. $(4.11))$

$$
\bar{w}_{*}=x\left(\tau \mid z_{0}, u_{\bar{x}_{0}, \bar{\xi}(\cdot)}(\cdot)\right),
$$

где

$$
z_{0} \in X_{0}^{\eta(\tau)}\left(\tau \mid \bar{x}_{0}, \bar{\xi}(\cdot), u_{\bar{x}_{0}, \bar{\xi}(\cdot)}(\cdot)\right) .
$$

Последнее включение, по определению множества из его правой части, означает, что существует $\zeta_{0}(\cdot) \in \Xi^{\eta(\tau)}$ такое, что пара $\left(z_{0}, \zeta_{0}(\cdot)\right)$ совместима с тройкой $\left(\bar{x}_{0}, \bar{\xi}(\cdot), u_{\bar{x}_{0}, \bar{\xi}(\cdot)}(\cdot)\right)$ до момента $\tau$. Отсюда следуют два вывода: во-первых, по определяющему свойству пакета программ

$$
u_{z_{0}, \zeta_{0}(\cdot)}(t)=u_{\bar{x}_{0}, \bar{\xi}(\cdot)}(t) \quad\left(t \in\left[t_{0}, \tau\right]\right)
$$


и, следовательно (см. (6.22)),

$$
\bar{w}_{*}=x\left(\tau \mid z_{0}, u_{z_{0}, \zeta_{0}(\cdot)}(\tau)\right) ;
$$

во-вторых, по следствию 3.1 множества

$$
\begin{array}{ll}
Z_{0}(\tau)=X_{0}^{\eta(\tau)}\left(\tau \mid z_{0}, \zeta_{0}(\cdot), u_{z_{0}, \zeta_{0}(\cdot)}(\cdot)\right), & Z(\tau)=X^{\eta(\tau)}\left(\tau \mid z_{0}, \zeta_{0}(\cdot), u_{z_{0}, \zeta_{0}(\cdot)}(\cdot)\right), \\
E_{0}(\tau)=X_{0}^{\eta(\tau)}\left(\tau \mid \bar{x}_{0}, \bar{\xi}(\cdot), u_{\bar{x}_{0}, \bar{\xi}(\cdot)}(\cdot)\right), & E(\tau)=X^{\eta(\tau)}\left(\tau \mid \bar{x}_{0}, \bar{\xi}(\cdot), u_{\bar{x}_{0}, \bar{\xi}(\cdot)}(\cdot)\right)
\end{array}
$$

попарно равны друг другу:

$$
Z_{0}(\tau)=E_{0}(\tau), \quad Z(\tau)=E(\tau) .
$$

Зададим $\zeta(\cdot) \in \Xi$ условием:

$$
\zeta(t)= \begin{cases}\zeta_{0}(t) & \text { при } t \in\left[t_{0}, \tau\right], \\ 0 & \text { при } t \in(\tau, \vartheta] .\end{cases}
$$

Так как $\zeta_{0}(\cdot) \in \Xi^{\eta(\tau)}$ и $\eta(\tau) \leqslant \eta(\tau+\delta) \leqslant \bar{h}$, то $\zeta(\cdot) \in \Xi^{\eta(\tau+\delta)} \subset \Xi^{\bar{h}}$. Ясно, что пара $\left(z_{0}, \zeta(\cdot)\right)$ совместима с тройкой $\left(z_{0}, \zeta_{0}(\cdot), u_{z_{0}, \zeta_{0}(\cdot)}(\cdot)\right)$ до момента $\tau$, поэтому

$$
u_{z_{0}, \zeta(\cdot)}(t)=u_{z_{0}, \zeta_{0}(\cdot)}(t) \quad\left(t \in\left[t_{0}, \tau\right]\right) .
$$

Определим нужную нам программу $v(\cdot)$ условием

$$
v(\cdot)=u_{z_{0}, \zeta(\cdot)}(t)
$$

Введем движение

$$
\bar{w}_{*}(\cdot)=x\left(\cdot \mid z_{0}, v(\cdot)\right) .
$$

Заметим, что в силу $(6.22)$ и (6.28)

$$
\bar{w}_{*}(\tau)=e\left(\widehat{x}_{*}^{[\nu]}\right) .
$$

Таким образом, движение $\bar{w}_{*}(\cdot)$ сдвигает точку $\bar{w}_{*}=e\left(\bar{x}_{*}^{[\nu]}\right) \in E(\tau)$ от момента $\tau$ до момента $\tau+\delta$ в силу программы $v(\cdot)$. Ясно, что пара $\left(z_{0}, \zeta(\cdot)\right)$ совместима с тройкой $\left(z_{0}, \zeta(\cdot), v(\cdot)\right)$ до момента $\tau+\delta$, поэтому, в соответствии с определением множества $E(\tau+\delta)(4.18)$,

$$
\bar{w}_{*}(\tau+\delta) \in E(\tau+\delta) .
$$

Ниже будут использованы следующие равенства, вытекающие из (6.26) и (6.29):

$$
\begin{gathered}
E(\tau)=Z(\tau)=X^{\eta(\tau)}\left(\tau \mid z_{0}, \zeta_{0}(\cdot), v(\cdot)\right), \\
E_{0}(\tau)=Z_{0}(\tau)=X_{0}^{\eta(\tau)}\left(\tau \mid z_{0}, \zeta_{0}(\cdot), v(\cdot)\right)
\end{gathered}
$$

Теперь, наряду с элементом $\bar{w}_{*}=e\left(\bar{x}_{*}^{[\nu]}\right)$, рассмотрим элемент $e(\widehat{x})$, отвечающий произвольной точке $\widehat{x} \in \widehat{E}$. Так как $e(\widehat{x}) \in E(\tau)=Z(\tau)$, то

$$
e(\widehat{x})=x\left(\tau \mid w_{0}(\widehat{x}), v(\cdot)\right),
$$


где $w_{0}(\widehat{x}) \in E_{0}(\tau)=Z_{0}(\tau)$. Положим

$$
w(\cdot \mid \widehat{x})=x\left(\cdot \mid w_{0}(\widehat{x}), v(\cdot)\right) .
$$

Заметим, что в силу (6.34)

$$
e(\widehat{x})=w(\tau \mid \widehat{x}) .
$$

Таким образом, движение $w(\cdot \mid \widehat{x})$ сдвигает точку $e(\widehat{x})$ от момента $\tau$ до момента $\tau+\delta$ при помощи программы $v(\cdot)$. Отметим, что определенное ранее движение $\bar{w}_{*}(\cdot)$ (как видно из (6.30) и (6.31)) может быть записано как

$$
\bar{w}_{*}(\cdot)=w\left(\cdot \mid \bar{x}_{*}^{[\nu]}\right) .
$$

Поэтому включение (6.32) мы будем ниже использовать в форме

$$
w\left(\tau+\delta \mid \bar{x}_{*}^{[\nu]}\right) \in E(\tau+\delta) .
$$

Далее будет показано, что для “достаточной” части элементов $\widehat{x} \in \widehat{E}^{\nu}(\tau)$ также выполняется подобное включение: $w\left(\tau+\delta \mid \widehat{x}_{*}^{[\nu]}\right) \in E(\tau+\delta)$. При доказательстве этого будет использовано следующее замечание, справедливое для произвольного $\widehat{x} \in \widehat{E}^{\nu}(\tau)$. Тот факт, что $w(\cdot \mid \widehat{x})=x\left(\cdot \mid w_{0}(\widehat{x}), v(\cdot)\right)$ при некотором $w_{0}(\widehat{x}) \in$ $Z_{0}(\tau)$ (см. $\left.(6.35)\right)$, вместе с (6.33) означает, что существует $\xi(\cdot \mid \widehat{x}) \in \Xi^{\eta(\tau)}$ такое, что пара $\left(w_{0}(\widehat{x}), \xi(\cdot \mid \widehat{x})\right)$ совместима с тройкой $\left(z_{0}, \zeta_{0}(\cdot), v(\cdot)\right)$, т.е.

$$
s(w(\cdot \mid \widehat{x}))+\xi(t \mid \widehat{x})=s\left(x\left(t \mid z_{0}, v(\cdot)\right)\right)+\zeta_{0}(t) \quad\left(t \in\left[t_{0}, \tau\right]\right)
$$

или, с учетом (6.37), (6.30) и (6.27)

$$
s(w(\cdot \mid \widehat{x}))+\xi(t \mid \widehat{x})=s\left(w\left(t \mid \bar{x}_{*}^{[\nu]}\right)\right)+\zeta(t) \quad\left(t \in\left[t_{0}, \tau\right]\right) .
$$

Третий этап. Рассмотрим произвольную точку $\widehat{x} \in \widehat{E}^{\nu}(\tau)$. Согласно (6.9), $\widehat{x}=\widehat{x}_{*}^{[\nu]}$ для некоторой точки $\widehat{x}_{*} \in \widehat{E}(\tau)$. Для упрощения обозначений введенное ранее движение $\widehat{x}_{*}^{[\nu]}(\cdot)$ под действием программы $u_{\tau+\delta}(\cdot)$, проходящее в момент $\tau$ через точку $\widehat{x}=\widehat{x}_{*}^{[\nu]} \in \widehat{E}^{\nu}(\tau)$ (см. (6.3) и (6.8)), будем также записывать как $z(\cdot \mid \widehat{x})$. Таким образом,

$$
z(\cdot \mid \widehat{x})=\widehat{x}_{*}^{[\nu]}(\cdot) \quad\left(\widehat{x}=\widehat{x}_{*}^{[\nu]} \in \widehat{E}^{\nu}(\tau)\right) .
$$

Для $t \in[\tau, \tau+\delta]$ положим:

$$
d(t)=\sum_{\widehat{x} \in \widehat{E}^{\nu}(\tau)}|z(t \mid \widehat{x})-w(t \mid \widehat{x})|^{2} .
$$

Так как при каждом $\widehat{x} \in \widehat{E}^{\nu}(\tau)$ имеем $z(\tau \mid \widehat{x})=\widehat{x}$ и $w(\tau \mid \widehat{x})=e(\widehat{x})($ см. $(6.36))$ и для $e(\widehat{x})$ выполняется оценка $(6.21)$, то

$$
\begin{aligned}
d(\tau) & =\sum_{\widehat{x} \in \widehat{E}^{\nu}(\tau)}|\widehat{x}-e(\widehat{x})|^{2} \\
& \leqslant \sum_{\widehat{x} \in \widehat{E}^{\nu}(\tau)} \operatorname{dist}(\widehat{x}, E(\tau))^{2}+\left|\widehat{E}^{\nu}(\tau)\right| \beta \\
& \leqslant \sum_{\widehat{x} \in \widehat{E}^{\nu}(\tau)} \operatorname{dist}(\widehat{x}, E(\tau))^{2}+\left|X_{0}^{\nu}\right| \beta \\
& =\rho^{2}\left(\widehat{E}^{\nu}(\tau), E(\tau)\right)+\left|X_{0}^{\nu}\right| \beta
\end{aligned}
$$


(см. (4.7)). По определению сужение функции $z(\cdot \mid \widehat{x})$ на $[\tau, \tau+\delta]$ есть решение на этом отрезке дифференциального уравнения $\dot{x}(t)=f\left(t, x(t), u_{*}\right)$ с начальным условием $z(\tau \mid \widehat{x})=\widehat{x}$, а сужение функции $w(\cdot \mid \widehat{x})$ на $[\tau, \tau+\delta]$ есть решение на этом отрезке дифференциального уравнения $\dot{x}(t)=f(t, x(t), v(t))$ с начальным условием $w(\tau \mid \widehat{x})=e(\widehat{x})$. Поэтому для $t \in[\tau, \tau+\delta]$

$$
\begin{aligned}
d(t) & =\sum_{\widehat{x} \in \widehat{E}^{\nu}(\tau)}\left|\widehat{x}-e(\widehat{x})+\int_{\tau}^{t}\left[f\left(s, z(s \mid \widehat{x}), u_{*}\right)-f(s, w(s \mid \widehat{x}), v(s))\right] d s\right|^{2} \\
& =\sum_{\widehat{x} \in \widehat{E}^{\nu}(\tau)}|\widehat{x}-e(\widehat{x})|^{2}+a_{1}(t)+a_{2}(t) \\
& =d(\tau)+a_{1}(t)+a_{2}(t)
\end{aligned}
$$

где

$$
\begin{aligned}
& a_{1}(t)=2 \sum_{\widehat{x} \in \widehat{E}^{\nu}(\tau)} \int_{\tau}^{t}\left\langle\widehat{x}-e(\widehat{x}), f\left(s, z(s \mid \widehat{x}), u_{*}\right)-f(s, w(s \mid \widehat{x}), v(s))\right\rangle d s, \\
& a_{2}(t)=\sum_{\widehat{x} \in \widehat{E}^{\nu}(\tau)}\left|\int_{\tau}^{t}\left[f\left(s, z(s \mid \widehat{x}), u_{*}\right)-f(s, w(s \mid \widehat{x}), v(t))\right] d s\right|^{2} .
\end{aligned}
$$

Учитывая, что движения $z(\cdot \mid \widehat{x})$ и $w(\cdot \mid \widehat{x})$ липшицевы с константой Липшица $K_{f}$, а нормы их значений ограничены константой $K_{X}$, и используя липшицевость на множестве $X$ функции $f(\cdot)$ по фазовой переменной с константой Липшица $L$ (см. условие $(\mathrm{A} 2))$, получаем:

$$
a_{1}(t)=2 \sum_{\widehat{x} \in \widehat{E}^{\nu}(\tau)} \int_{\tau}^{t}\left\langle\widehat{x}-e(\widehat{x}), f\left(s, \widehat{x}, u_{*}\right)-f(s, e(\widehat{x}), v(s))\right\rangle d s+a_{11}(t)
$$

где

$$
\begin{aligned}
a_{11}(t)= & 2 \sum_{\widehat{x} \in \widehat{E}^{\nu}(\tau)} \int_{\tau}^{t}\left\langle\widehat{x}-e(\widehat{x}), f\left(s, z(s \mid \widehat{x}), u_{*}\right)-f\left(s, \widehat{x}, u_{*}\right)\right\rangle d s \\
& +2 \sum_{\widehat{x} \in \widehat{E}^{\nu}(\tau)} \int_{\tau}^{t}\left\langle\widehat{x}-e(\widehat{x}), f\left(s, w(s \mid \widehat{x}), u_{*}\right)-f(s, e(\widehat{x}), v(s))\right\rangle d s \\
\leqslant & 2 \sum_{\widehat{x} \in \widehat{E}^{\nu}(\tau)} \int_{\tau}^{t}|\widehat{x}-e(\widehat{x})| 2 L K_{f}(s-\tau) d s \\
\leqslant & 2 \sum_{\widehat{x} \in \widehat{E}^{\nu}(\tau)} \int_{\tau}^{t} 4 K_{X} L K_{f}(s-\tau) d s \\
\leqslant & \sum_{\widehat{x} \in \widehat{E}^{\nu}(\tau)} 4 K_{X} L K_{f} \delta^{2} \\
\leqslant & 4 K_{X} L K_{f}\left|X_{0}^{\nu}\right| \delta^{2} .
\end{aligned}
$$

Очевидно,

$$
a_{2}(t) \leqslant 4 K_{f}\left|X_{0}^{\nu}\right| \delta^{2}
$$


Продолжим (6.44):

$$
\begin{aligned}
a_{1}(t)=2 & \sum_{\widehat{x} \in \widehat{E}^{\nu}(\tau)} \int_{\tau}^{t}\left\langle\widehat{x}-e(\widehat{x}), f\left(\tau, \widehat{x}, u_{*}\right)-f(\tau, \widehat{x}, v(s))\right\rangle d s \\
& +a_{11}(t)+a_{12}(t)+a_{13}(t),
\end{aligned}
$$

где

$$
\begin{aligned}
a_{12}(t)= & 2 \sum_{\widehat{x} \in \widehat{E}^{\nu}(\tau)} \int_{\tau}^{t}\left\langle\widehat{x}-e(\widehat{x}), f\left(s, \widehat{x}, u_{*}\right)-f\left(\tau, \widehat{x}, u_{*}\right)\right\rangle d s \\
& -2 \sum_{\widehat{x} \in \widehat{E}^{\nu}(\tau)} \int_{\tau}^{t}\langle\widehat{x}-e(\widehat{x}), f(s, e(\widehat{x}), v(s))-f(\tau, e(\widehat{x}), v(s))\rangle d s \\
& \leqslant 2 \sum_{\widehat{x} \in \widehat{E}^{\nu}(\tau)} 2|\widehat{x}-e(\widehat{x})| \mu(\delta) \delta \\
= & 8 K_{X}\left|X_{0}^{\nu}\right| \mu(\delta) \delta \\
a_{13}(t)= & 2 \sum_{\widehat{x} \in \widehat{E}^{\nu}(\tau)} \int_{\tau}^{t}\langle\widehat{x}-e(\widehat{x}), f(s, \widehat{x}, v(s))-f(s, e(\widehat{x}), v(s))\rangle d s \\
\leqslant & 2 \sum_{\widehat{x} \in \widehat{E}^{\nu}(\tau)} L|\widehat{x}-e(\widehat{x})|^{2} \delta \\
= & 2 L d(\tau) \delta
\end{aligned}
$$

(здесь мы использовали первое равенство из (6.42)). Так как $u_{*}$ по определению минимизирует на $P$ функцию $\varphi(\cdot)$ вида (6.20), то первое слагаемое в правой части (6.47) неположительно и, таким образом,

$$
a_{1}(t) \leqslant a_{11}(t)+a_{12}(t)+a_{13}(t) .
$$

Подставляя эту оценку и оценку (6.46) в (6.43) и затем пользуясь оценками (6.45), (6.48) и (6.49), получаем:

$$
d(t) \leqslant d(\tau)(1+2 L \delta)+C\left|X_{0}^{\nu}\right|(\delta+\mu(\delta)) \delta,
$$

где

$$
C=4 K_{f}\left(K_{X} L+1\right)+8 K_{X} .
$$

Подставляя верхнюю оценку (6.42) для $d(\tau)$, приходим к окончательной оценке:

$$
\begin{aligned}
& d(t) \leqslant \bar{d}=\rho^{2}\left(\widehat{E}^{\nu}(\tau), E(\tau)\right)(1+2 L \delta)+\left|X_{0}^{\nu}\right|(1+2 L \delta) \beta+C\left|X_{0}^{\nu}\right|(\delta+\mu(\delta)) \delta \\
& (t \in[\tau, \tau+\delta]) .
\end{aligned}
$$

Четвертый этап. Перейдем к обоснованию заключительной верхней оценки для расстояния $\rho\left(\widehat{E}^{\nu}(\tau+\delta), E(\tau+\delta)\right)$. Как следует из (6.9), отображение $\widehat{x}_{* 0}^{\nu} \mapsto \widehat{x}_{*}^{[\nu]}$ из $\widehat{E}_{0}^{\nu}(\tau)$ (см. (6.18)) на $\widehat{E}^{\nu}(\tau)$ взаимно однозначно. Согласно (6.18), $\widehat{E}_{0}^{\nu}(\tau+\delta) \subset \widehat{E}_{0}^{\nu}(\tau)$, и, согласно (6.17), отображение $\widehat{x}_{* 0}^{\nu} \mapsto \widehat{x}_{*}^{[\nu]}(\tau+\delta)$ из $\widehat{E}_{0}^{\nu}(\tau+\delta)$ на $\widehat{E}^{\nu}(\tau+\delta)$ взаимно однозначно. Поэтому, выделив множество

$$
\widehat{E}_{\delta}^{\nu}(\tau)=\left\{\widehat{x}_{*}^{[\nu]} \in \widehat{E}^{\nu}(\tau): \widehat{x}_{0}^{\nu} \in \widehat{E}_{0}^{\nu}(\tau+\delta)\right\},
$$


получаем, что отображение $\widehat{x}_{*}^{[\nu]} \mapsto \widehat{x}_{*}^{[\nu]}(\tau+\delta)$ из $\widehat{E}_{\delta}^{\nu}(\tau) \subset E^{\nu}(\tau)$ на $\widehat{E}^{\nu}(\tau+\delta)$ взаимно однозначно. В обозначениях (6.40) данное отображение имеет вид $\widehat{x} \mapsto z(\tau+\delta \mid \widehat{x})$. Таким образом,

$$
\widehat{E}^{\nu}(\tau+\delta)=\left\{z(\tau+\delta \mid \widehat{x}): \widehat{x} \in \widehat{E}_{\delta}^{\nu}(\tau)\right\}
$$

Заметим, что в обозначениях (6.40) неравенство (6.19) принимает вид

$$
\left|s(z(t \mid \widehat{x}))-y_{\tau+\delta}(t)\right| \leqslant h+\sigma(\nu) \quad\left(t \in\left[t_{0}, \tau+\delta\right], \quad \widehat{x} \in \widehat{E}_{\delta}^{\nu}(\tau)\right)
$$

(при переходе от (6.19) к (6.53) также учтена взаимная однозначность отображения $\widehat{x}_{*}^{[\nu]} \mapsto \widehat{x}_{* 0}^{\nu}$ или, что то же, $\widehat{x} \mapsto z\left(t_{0} \mid \widehat{x}\right)$ из $\widehat{E}_{\delta}^{\nu}(\tau)$ на $\left.\widehat{E}_{0}^{\nu}(\tau+\delta)\right)$. Для $t \in[\tau, \tau+\delta]$ положим

$$
d_{\delta}(t)=\sum_{\widehat{x} \in \widehat{E}_{\delta}^{\nu}(\tau)}|z(t \mid \widehat{x})-w(t \mid \widehat{x})|^{2} .
$$

Так как $\widehat{E}_{\delta}^{\nu}(\tau+\delta) \subset \widehat{E}^{\nu}(\tau)$, то

$$
d_{\delta}(t) \leqslant d(t) \leqslant \bar{d}
$$

(см. (6.41) и (6.51)).

Рассмотрим (в качестве точки $\widehat{x})$ точку $\bar{x}_{*}^{[\nu]} \in E(\tau)$, которая аппроксимирует точку $\bar{x}_{*} \in \widehat{E}(\tau)$, выделенную в условии (ii). Выше было показано, что для движения $\bar{x}_{*}(\cdot)$ под действием программы $u_{\tau+\delta}(\cdot)$, проходящего в момент $\tau$ через точку $\bar{x}_{*}$, справедливо включение $\bar{x}_{*}(\tau+\delta) \in \widehat{E}(\tau+\delta)$ (см. (6.14)). Поэтому для аппроксимируюшего движения $\bar{x}_{*}^{[\nu]}(\cdot)=z\left(\cdot \mid \bar{x}_{*}^{[\nu]}\right)$ под действием программы $u_{\tau+\delta}(\cdot)$, проходящего в момент $\tau$ через интересующую нас точку $\bar{x}_{*}^{[\nu]}$, справедливо включение $z\left(\tau+\delta \mid \bar{x}_{*}^{[\nu]}\right) \in \widehat{E}^{\nu}(\tau+\delta)$. Значит, в соответствии с (6.52), $\bar{x}_{*}^{[\nu]} \in \widehat{E}_{\delta}^{\nu}(\tau)$. Поэтому, как следует из определения $d_{\delta}(t)(6.54)$,

$$
\left|z\left(t \mid \bar{x}_{*}^{[\nu]}\right)-w\left(t \mid \bar{x}_{*}^{[\nu]}\right)\right|^{2} \leqslant d_{\delta}(t) \quad(t \in[\tau, \tau+\delta]) .
$$

Аналогично заключаем, что для всякого $\bar{x} \in \widehat{E}_{\delta}^{\nu}(\tau)$

$$
|z(t \mid \bar{x})-w(t \mid \bar{x})|^{2} \leqslant d_{\delta}(t) \quad(t \in[\tau, \tau+\delta]) .
$$

Используем эти оценки, а также ряд полученных ранее соотношений, чтобы установить, что

$$
w(\tau+\delta \mid \widehat{x}) \in E(\tau+\delta) \quad\left(\widehat{x} \in \widehat{E}_{\delta}^{\nu}(\tau)\right) .
$$

Возьмем произвольный элемент $\widehat{x} \in \widehat{E}_{\delta}^{\nu}(\tau)$. Для доказательства (6.58) следует показать, что существует $\omega(\cdot \mid \widehat{x}) \in \Xi^{\eta(\tau+\delta)}$ такое, что пара $\left(w\left(t_{0} \mid \widehat{x}\right), \omega(\cdot \mid \widehat{x})\right)$ совместима с тройкой $\left(z_{0}, \zeta(\cdot), v(\cdot)\right)=\left(w\left(t_{0} \mid \bar{x}_{*}^{[\nu]}\right), \zeta(\cdot), v(\cdot)\right)$ до момента $\tau+\delta$, т.е.

$$
s(w(\cdot \mid \widehat{x}))+\omega(t \mid \widehat{x})=s\left(w\left(t \mid \widehat{x}_{*}^{[\nu]}\right)\right)+\zeta(t)
$$

при всех $t \in\left[t_{0}, \tau+\delta\right]$. Положим $\omega(t \mid \widehat{x})=\xi(t \mid \widehat{x})$ при $t \in\left[t_{0}, \tau\right]$. Тогда по $(6.39)$ неравенство (6.59) верно при всех $t \in\left[t_{0}, \tau\right]$, причем

$$
|\omega(t \mid \widehat{x})|=|\xi(t \mid \widehat{x})| \leqslant \eta(\tau) \quad\left(t \in\left[t_{0}, \tau\right]\right),
$$


так как $\xi(\cdot \mid \widehat{x}) \in \Xi^{\eta(\tau)}$. Для завершения доказательства справедливости (6.59) при всех $t \in\left[t_{0}, \tau+\delta\right]$, с учетом того, что $\zeta(t)=0$ при $t \in(\tau, \tau+\delta]$ (см. (6.27)), нам осталось показать, что

$$
\left|s(w(\cdot \mid \widehat{x}))-s\left(w\left(t \mid \bar{x}_{*}^{[\nu]}\right)\right)\right| \leqslant \eta(\tau+\delta)
$$

при всяком $t \in(\tau, \tau+\delta]$. Возьмем произвольный такой момент $t$. Имеем:

$$
\begin{aligned}
\left|s(w(\cdot \mid \widehat{x}))-s\left(w\left(t \mid \bar{x}_{*}^{[\nu]}\right)\right)\right| \leqslant \mid s & (w(\cdot \mid \widehat{x}))-s(z(t \mid \widehat{x})) \mid \\
& +\left|s(z(t \mid \widehat{x}))-y_{\tau+\delta}(t)\right| \\
& +\left|y_{\tau+\delta}(t)-s\left(z\left(t \mid \bar{x}_{*}^{[\nu]}\right)\right)\right| \\
& +\left|s\left(z\left(t \mid \bar{x}_{*}^{[\nu]}\right)\right)-s\left(w\left(t \mid \bar{x}_{*}^{[\nu]}\right)\right)\right| .
\end{aligned}
$$

Оценим сверху каждое из четырех слагаемых из правой части. Для первого слагаемого (6.57) и (6.51) влекут оценки

$$
|s(z(t \mid \bar{x}))-s(w(t \mid \bar{x}))| \leqslant \sigma\left(d_{\delta}(t)^{1 / 2}\right) \leqslant \sigma\left(\bar{d}^{1 / 2}\right)
$$

(напомним, что $\sigma(\cdot)$ - модуль непрерывности функции $s(\cdot)$ на множестве $X$ ); для второго и третьего слагаемых в соответствии с (6.53) имеем

$$
\begin{aligned}
\left|s(z(t \mid \widehat{x}))-y_{\tau+\delta}(t)\right| & \leqslant h+\sigma(\nu), \\
\left|y_{\tau+\delta}(t)-s\left(z\left(t \mid \bar{x}_{*}^{[\nu]}\right)\right)\right| & \leqslant h+\sigma(\nu) ;
\end{aligned}
$$

для четвертого слагаемого из (6.56) и (6.51) следует:

$$
\left|s\left(z\left(t \mid \bar{x}_{*}^{[\nu]}\right)\right)-s\left(w\left(t \mid \bar{x}_{*}^{[\nu]}\right)\right)\right| \leqslant \sigma\left(d_{\delta}(t)^{1 / 2}\right) \leqslant \sigma\left(\bar{d}^{1 / 2}\right) .
$$

Подставляя полученные четыре оценки в (6.61), получаем:

$$
\left|s(w(\cdot \mid \widehat{x}))-s\left(w\left(t \mid \widehat{x}_{*}^{[\nu]}\right)\right)\right| \leqslant 2\left(\sigma\left(\bar{d}^{1 / 2}\right)+h+\sigma(\nu)\right) \leqslant \eta(\tau+\delta) ;
$$

последнее нервенство следует из того, что по условию леммы (см. (4.15))

$$
\eta(\tau+\delta) \geqslant \max \left\{\eta(\tau), 2\left(\sigma\left(\bar{d}^{1 / 2}\right)+h+\sigma(\nu)\right)\right\}
$$

где $\bar{d}$ задано по (6.51), (6.50) (см. (4.15), (4.13), (4.14)). Нужное неравенство (6.60) установлено. Тем самым показано (6.58).

Теперь легко приходим к окончательной оценке

$$
\rho^{2}\left(\widehat{E}^{\nu}(\tau+\delta), E(\tau+\delta)\right) \leqslant \bar{d}
$$

(см. (4.19)). Действительно, (6.58) гарантирует, что

$$
\operatorname{dist}(z(\tau+\delta \mid \widehat{x}), E(\tau+\delta)) \leqslant|z(\tau+\delta \mid \widehat{x})-w(\tau+\delta \mid \widehat{x})|
$$


при всяком $\widehat{x} \in \widehat{E}_{\delta}^{\nu}(\tau)$. Так как при $\widehat{x}$, пробегающем $\widehat{E}_{\delta}^{\nu}(\tau)$, точки $z(\tau+\delta \mid \widehat{x})$ покрывают множество $\widehat{E}^{\nu}(\tau+\delta)$ (см. (6.52)), то

$$
\begin{aligned}
\rho^{2}\left(\widehat{E}^{\nu}(\tau+\delta), E(\tau+\delta)\right) & =\sum_{\widehat{z} \in \widehat{E}^{\nu}(\tau+\delta)} \operatorname{dist}(\widehat{z}, E(\tau+\delta))^{2} \\
& =\sum_{\widehat{x} \in \widehat{E}_{\delta}^{\nu}(\tau)} \operatorname{dist}(z(\tau+\delta \mid \widehat{x}), E(\tau+\delta))^{2} \\
& \leqslant \sum_{\widehat{x} \in \widehat{E}_{\delta}^{\nu}(\tau)}|z(\tau+\delta \mid \widehat{x})-w(\tau+\delta \mid \widehat{x})|^{2} \\
& =d_{\delta}(\tau+\delta) \leqslant \bar{d}
\end{aligned}
$$

(последние равенство и неравенство обеспечены (6.54) и (6.55)). Оценка (6.62) получена. Доказательство леммы 4.3 закончено.

\section{Заключение}

В работе предложен подход к исследованию задач позиционного управления с неполной информацией, основанный на адекватной программной конструкции - пакетах программ. Подход реализован в достаточно ограниченной ситуации, когда управляемая система не подвержена действию неопределенных динамических помех, а задача управления имеет простейший вид: навести систему на фиксированное целевое множество в фиксированный момент времени. Такие, упрощенные, условия выбраны с тем, чтобы выделить, прежде всего, эффект неполноты информации. С другой стороны, произвольность динамики системы делает рассмотренную ситуацию достаточно характерной, материал работы может составить основу для дальнейших исследований.

Представляется, что дальнейшие исследования могут развиваться по двум направлениям, условно говоря, "вширь" и “вглубь". Продвижения "вширь" могут быть нацелены на распространение основного результата работы - утверждения об эквивалентности задачи позиционного наведения и задачи пакетного наведения - на другие содержательные случаи, например, на случай наведения системы на целевое множество не позже заданного момента времени, на случай наведения на целевое множество при фазовых ограничениях, на случай оптимизации заданного (скажем, интегрально-терминального) показателя качества и т.д. Наиболее трудным - и одновременно наиболее интересным направлением обобщений может стать исследование, с позиций предложенного подхода, дифференциально-игровых задач с неполной информацией. Для этого класса задач, отягченных присутствием неопределенных динамических помех на входе управляемой системы, представляется важным дать адекватное определение игрового (обобщенного) пакета программ - некоторого "гибрида" пакета программ, рассмотренного в настоящей работе, и квазистратегии как неупреждающего программного оператора из теории позиционных дифференциальных игр [12]. Движение в этом направлении можно было бы начать с изучения частных случаев, близких, в том или ином смысле, к ситуации неигрового управления (например, со случаев типа “стабильной дорожки" [6], в которых разрешающая квазистратегия имеет заранее известный вид). 
Для продвижений “вглубь” также видится несколько направлений. Одно из них - выявление конструктивных условий разрешимости задачи пакетного наведения. В этой связи целесообразно было бы обращение к конкретным классам управляемых систем, например, к линейным системам с линейными сигналами о состояниях, выпуклыми - либо конечными - целевыми множествами и такими же множествами допустимых начальных состояний. Другая интересная задача - разработка численно реализуемых алгоритмов управления с ограниченной памятью. Заметим, что в случае конечного множества допустимых начальных состояний описанные в настоящей работе стратегии экстремального сдвига не требуют увеличения размеров конечной аппроксимирующей сетки на этом множестве (роль такой сетки играет само множество); в этом смысле стратегии экстремального сдвига имеют ограниченную память и могут выступать в качестве реализуемых алгоритмов управления; была бы полезна конкретизация их в этом качестве. В общей ситуации, когда множество допустимых начальных состояний не конечно, описанные стратегии экстремального сдвига требуют при своей реализации большого (в пределе бесконечного) объема памяти. В этой ситуации актуальным становится вопрос об альтернативных, реализуемых, вариантах стратегий экстремального сдвига. Такого рода исследование естественно было бы ограничить классами управляемых систем, например, линейными системами с линейными сигналами о состояниях.

\section{Список литературы}

[1] Л. С. Понтрягин, В. Г. Болтянский, Р. В. Гамкрелизде, Е. Ф. Мищенко, Математическая теория оптимальных прочессов, Физматгиз, М., 1961.

[2] Р. Айзекс, Дифференциалъные игры, Мир, М., 1967.

[3] Л. С. Понтрягин, "О линейных дифференциальных играх. II", Докл. АН СССР, 175:4 (1967), 764-766.

[4] Л. С. Понтрягин, “Линейная дифференциальная игра убегания”, Сборник статей, посвященных И.М. Виноградову к его 80-летию, Тр. МИАН, 112, 1971, 30-63.

[5] Н.Н. Красовский, Игровые задачи о встрече движений, Наука, М., 1970.

[6] Н. Н. Красовский, А. И. Субботин, Позиционные дифференциальные игры, Наука, M., 1974.

[7] Н.Н. Красовский, Управление динамической системой, Наука, М., 1985.

[8] N. N. Krasovskii, A. I. Subbotin, Game-theoretical control problems, Springer-Verlag, New York, 1988.

[9] A. N. Krasovskii, N. N. Krasovskii, Control under lack of information, Systems Control Found. Appl., Birkhäuser, Boston, MA, 1995.

[10] А. И. Субботин, Минимакснье неравенства и уравнения Гамильтона-Якоби, Наука, М., 1991.

[11] A. I. Subbotin, Generalized solutions of first-order PDEs: The dynamical optimization perspective, Systems Control Found. Appl., Birkhäuser, Boston, MA, 1995.

[12] А. И. Субботин, А. Г. Ченцов, Оптимизация гарантии в задачах управления, Наука, М., 1981.

[13] А. В. Кряжимский, С. Д. Филиппов, “Об игровой задаче наведения для двух точек на плоскости в условиях неполной информации”, Задачи управления при неполной информации, УНЦ АН СССР, Свердловск, 1976, 62-77. 
[14] В.Е. Пак, “Задача наведения с неполной информацией”, Изв. АН CCCP. Сер. техн. киберн., 1976, № 4, 29-36.

[15] В. Д. Батухтин, "Об одной игровой задаче наведения с неполной информацией”, Прикл. матем. и мех., 44:4 (1980), 595-601.

[16] Н.Н. Красовский, Ю. С. Осипов, “Задача управления с неполной информацией”, Механ. тверд. тела, 1973, № 4, 5-14.

[17] А.В. Кряжимский, “Дифференциальная игра сближения в условиях неполной информации о системе", Укр. матем. журн., 27:4 (1975), 521-526.

[18] А.В. Кряжимский, "Альтернатива в линейной игре сближения-уклонения с неполной информацией”, Докл. АН СССР, 230:4 (1976), 773-776.

[19] А. А. Крекнин, А. И. Субботин, "Игровая задача преследования в условиях неполной фазовой информации о преследуемой системе", Дифференииалъные системь управления, Тр. Ин-та матем. и мех. УНЦ АН СССР, 26, УНЦ АН СССР, Свердловск, 1979, 21-33.

[20] А.Б. Куржанский, "О синтезе управлений по результатам измерений", Прикл. матем. и мех., 68:4 (2004), 547-563.

[21] Yu. S. Osipov, A.V. Kryazhimskii, Inverse problems for ordinary differential equations: dynamical solutions, Gordon \& Breach, Basel, 1995.

[22] Ю. С. Осипов, Ф. П. Васильев, М. М. Потапов, Основы метода динамической регуляризации, Изд-во МГУ, М., 1999.

[23] A. V. Kryazhimskii, Yu. S. Osipov, "On positional calculation of $\Omega$-normal controls in dynamical systems", Problems Control Inform. Theory, 13:6 (1984), 425-436.

[24] F. Fagnani, V. Maksimov, L. Pandolfi, "A recursive deconvolution approach to disturbance reduction", IEEE Trans. Automat. Control, 49:6 (2004), 907-921.

[25] E. Roxin, "Axiomatic approach in differential games", J. Optim. Theory Appl., 3 (1969), 153-163.

[26] C. Ryll-Nardzewski, "A theory of pursuit and evasion", Advances in game theory, Ann. of Math. Stud., 52, Princeton Univ. Press, Princeton, NJ, 1964, 113-126.

[27] А. Н. Тихонов, "О функциональных уравнениях типа Вольтерра и их применении к некоторым задачам математической физики", Бюлл. МГУ, секц. А, 1938, № 8 , $1-25$.

[28] А.Н. Тихонов, В.Я. Арсенин, Методъ решения некорректных задач, Наука, М., 1979.

[29] Дж. Варга, Оптимальное управление дифференциальными и функииональными уравнениями, Наука, М., 1977.

[30] А.Б. Куржанский, Управление и наблюдение в условиях неопределенности, Наука, М., 1977.

Ю. С. Осипов (Yu. S. Osipov)

Математический институт им. В. А. Стеклова РАН
Поступила в редакцию

04.05.2006 